\title{
Blood platelet rheology in venules and arterioles
}

Citation for published version (APA):

Woldhuis, B. (1993). Blood platelet rheology in venules and arterioles. [Doctoral Thesis, Maastricht University]. Rijksuniversiteit Limburg. https://doi.org/10.26481/dis.19931223bw

Document status and date:

Published: 01/01/1993

DOI:

10.26481/dis.19931223bw

Document Version:

Publisher's PDF, also known as Version of record

\section{Please check the document version of this publication:}

- A submitted manuscript is the version of the article upon submission and before peer-review. There can be important differences between the submitted version and the official published version of record.

People interested in the research are advised to contact the author for the final version of the publication, or visit the DOI to the publisher's website.

- The final author version and the galley proof are versions of the publication after peer review.

- The final published version features the final layout of the paper including the volume, issue and page numbers.

Link to publication

\footnotetext{
General rights rights.

- You may freely distribute the URL identifying the publication in the public portal. please follow below link for the End User Agreement:

www.umlib.nl/taverne-license

Take down policy

If you believe that this document breaches copyright please contact us at:

repository@maastrichtuniversity.nl

providing details and we will investigate your claim.
}

Copyright and moral rights for the publications made accessible in the public portal are retained by the authors and/or other copyright owners and it is a condition of accessing publications that users recognise and abide by the legal requirements associated with these

- Users may download and print one copy of any publication from the public portal for the purpose of private study or research.

- You may not further distribute the material or use it for any profit-making activity or commercial gain

If the publication is distributed under the terms of Article $25 \mathrm{fa}$ of the Dutch Copyright Act, indicated by the "Taverne" license above, 


\section{BLOOD PLATELET RHEOLOGY}

IN

VENULES AND ARTERIOLES 


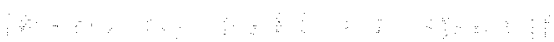

. 


\title{
BLOOD PLATELET RHEOLOGY \\ IN \\ VENULES AND ARTERIOLES
}

\author{
Proefschrift
}

ter verkrijging van de graad van doctor

aan de Rijksuniversiteit Limburg te Maastricht, op gezag van de Rector Magnificus, Prof. Dr. H. Philipsen, volgens het besluit van het College van Dekanen, in het openbaar te verdedigen op donderdag, 23 december 1993 om 16.00 uur

door

Berendje Woldhuis

geboren op 7 oktober 1957 te Veendam 


\section{Promotores:}

Prof.Dr. G.J. Tangelder

Prof.Dr. R.S. Reneman

\section{Co-promotor:}

Dr. D.W. Slaaf

\section{Beoordelingscommissie:}

Prof.Dr. H.A.J. Struljker Boudier, voorzitter Prof.Dr.Ir. T. Arts, Universiteit Twente Prof.Dr. B. Bouma, Universiteit Utrecht Prof.Dr. P.W. de Leeuw

Prof.Dr. R.F.A. Zwaal 
Aan mijn ouders

aan Olaf, Anne en Erik 
CIP-DATA KONINKLIJKE BIBLIOTHEEK DEN HAAG

Woldhuis, Berendje

Blood platelet rheology in venules and arterioles/

Berendje Woldhuis.-[S.I. : s.n.]

Thesis Maastricht. - With ref. - With summary in Dutch ISBN 90-9006692-6

Subject headings: platelets /rheology.

Het verschijnen van dit proefschrift werd mede mogelijk gemaakt door steun van:

- Stichting Wetenschappelijk Durrer-Fonds

- Nederlandse Hartstichting

Druk: ICG Printing, Dordrecht 


\section{CONTENTS}

\section{Chapter 1}

General introduction

Chapter 2

Platelet and blood rheology: a short overview

2.1 General fluid dynamics

2.2 Whole blood viscosity

2.3 Microcirculation

2.4 Dextrans and blood rheology

Chapter 3

Methodological survey

Chapter 4

Concentration profile of blood platelets

differ in arterioles and venules

4.1 Introduction 41

4.2 Materials and methods $\quad 42$

4.3 Results 46

$\begin{array}{ll}4.4 \text { Discussion } & 50\end{array}$

4.5 Appendix 54

$\begin{array}{lll}4.6 & \text { Summary } & 57\end{array}$

\section{Chapter 5}

Influence of dextrans on platelet distribution in arterioles and venules $\quad 59$

5.1 Introduction 59

5.2 Materials and methods $\quad 60$

5.3 Results 66

$\begin{array}{lll}5.4 & \text { Discussion } & 72\end{array}$

$\begin{array}{lll}5.5 & \text { Summary } & 76\end{array}$ 


\section{Chapter 6}

Velocity profiles in rabbit mesenteric venules in vivo

6.1 Introduction

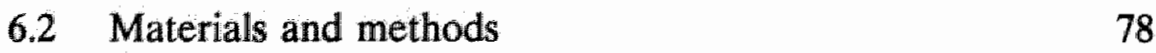

$\begin{array}{lll}6.3 \text { Results } & 85\end{array}$

6.4 Discussion 91

$\begin{array}{lll}6.5 & \text { Summary } & 96\end{array}$

\section{Chapter 7}

Platelet Fåhraeus effect in arterioles and venules as calculated from measured velocity profiles

and concentration distributions $\quad 99$

$\begin{array}{lll}7.1 & \text { Introduction } & 99\end{array}$

$\begin{array}{lll}7.2 & \text { Methods } & 100\end{array}$

$\begin{array}{lll}7.3 & \text { Results } & 105\end{array}$

$\begin{array}{lll}7.4 & \text { Discussion } & 109\end{array}$

$\begin{array}{ll}7.5 \text { Appendix } & 114\end{array}$

$\begin{array}{lll}7.6 & \text { Summary } & 115\end{array}$

Chapter 8

General discussion 117

$\begin{array}{ll}\text { References } & 123\end{array}$

$\begin{array}{ll}\text { Summary } & 131\end{array}$

$\begin{array}{ll}\text { Samenvatting } & 134\end{array}$

$\begin{array}{lr}\text { Publications } & 138\end{array}$

Dankwoord 140

$\begin{array}{ll}\text { Curriculum vitae } & 143\end{array}$ 


\section{GENERAL INTRODUCTION}

Small corpuscular blood elements other than red and white blood cells were recognized in the early days of the compound microscope, i.e. the first half of the 19th century (for review: Tocantins, 1948). The existence of platelets as a distinct blood element, however, was not firmly established before the end of that century. Bizzozero (1882) observed in the mesentery of mammals "morphologische Elemente einer dritten Art, neben den rothen und weissen Blutkörperchen". He called them "Blutplättchen" or blood platelets and showed that they adhere to an injured vessel wall to form a thrombus, which mainly consists of blood platelets. In the following years, the important role of blood platelets in hemostasis and thrombosis was established by several investigators (see reviews: Tocantins, 1948, Poole and French, 1961, Bergqvist, 1974; Sixma and Wester, 1977).

The microcirculation, comprising the smallest blood vessels, allows direct microscopic observation of vessels in which plasma and blood cells are flowing. The actual study of the behavior of individual platelets in vivo has been hampered by the excess of red blood cells. Without special staining techniques, platelets could only be observed if they were flowing close to the vessel wall, where the density of red blood cells is lowest (Bloch, 1968; Schmid-Schönbein and Zweifach, 1975). Fluorescence microscopy in combination with selective labelling of the blood platelets in vivo with the fluorochrome acridine red, yielded a clear microscopic image of individual platelets even when flowing in the vessel center (Tangelder et al, 1982a). With this labelling technique, aspects of the rheological behavior of platelets, such as their distribution (Tangelder et al, 1985), orientation (Teirlinck et al, 1984) and velocity profile (Tangelder et al, 1986) have been studied in arterioles. Arterioles are microvessels that deliver blood to capillaries, i.e. the smallest blood vessels in which exchange of oxygen and nutrients between blood and tissue takes place.

The microvessels which drain the blood from the capillaries, i.e. the venules, differ in many aspects from the feeding arterioles, e.g. flow pattern, in converging versus diverging at branch points, in permeability (De Clerck et al, 1985) and in their thromboembolic reaction to microinjury (oude 


\section{Chapter 1}

Egbrink et al, 1988). Few studies exist on the rheology of blood in venules. It was therefore the aim of this thesis to study flow behavior of blood platelets in venules in vivo, and to compare this behavior with that in arterioles and with results obtained from glass capillaries in vitro. Our studies were performed in the rabbit mesentery with the use of intravital fluorescence videomicroscopy.

As an introduction to the experimental chapters of this thesis, chapter 2 presents a short overview of the rheological aspects of blood in general and the flow behavior of platellets, red blood cells and leukocytes and their interactions in particular. Chapter 3 shortly introduces the techniques and methods used in the experiments described in the following chapters.

The first experimental chapter of this thesis (chapter 4) describes the differences in platelet distribution in arterioles and venules. The role of leukocyte rolling, a phenomenon only present in venules in this tissue preparation was explored.

Beside leukocytes, also red blood cells and endothelium may play a role in the difference in platelet distribution between arterioles and venules. Aggregation of red blood cells may influence the distribution of platelets. Interaction between platelets and the microvascular endothelium might also be involved. Dextrans, which are long sugar molecules can bind to the surface of red blood cells, endothelium and platelets. They influence red blood cell aggregation and, possibly, platelet-endothelium interaction. In chapter 5, we therefore investigate the influence of various dextrans on platelet distribution in arterioles and venules.

Another rheological factor that could be related to the observed difference in platelet distribution between arterioles and venules is the velocity profile which might be different in arterioles and venules. Therefore, in chapter 6 the velocity profile in venules is investigated in detail and compared with the velocity profiles in arterioles as determined previously (Tangelder et al, 1986).

In 1929, Fahraeus described that for blood flowing through tubes with diameters similar to those of arterioles and venules, red blood cell concentration within the tube decreases with decreasing tube diameter. This is caused by the fact that red blood cells tend to flow more in the center, which leads to a higher average travelling speed of the red blood cells than that of the suspending fluid. Mass conservation requires that such a higher velocity is compensated for by a reduced concentration of the red blood cells. The uneven distribution of platelets in arterioles and venules may lead to average travelling speeds different from those found by homogeneously distributed platelets. It may result in a platelet Fåhraeus effect or possibly 
an inverted Fåhraeus effect. In chapter 7 the tube platelet Fåhraeus effect in arterioles and venules is calculated from the measured velocity profiles (chapter 6) and concentration distributions (chapter 4) of the platelets.

Finally, chapter 8 contains a general discussion in which data obtained in the various experiments will be integrated and discussed. Summaries and appendices conclude this thesis. 


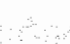

and

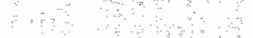




\section{PLATELET AND BLOOD RHEOLOGY: A SHORT OVERVIEW}

Blood platelets are small anucleate cells shed from the cytoplasm of megakaryocytes, i.e. the hemopoietic stem cell of platelets in the bone marrow. The fundamental need for platelets in hemostasis is obvious from what can be observed in patients with a low systemic platelet count: they have a prolonged bleeding time and easily develop purpura. Beside platelets, coagulation factors and endothelium are involved in the hemostatic process. Normally, circulating platelets come to all likelihood in frequent contact with the vessel wall (Tangelder et al, 1985, 1986), but they do not stick to the endothelium. In injured vessels, however, rapid activation and adherence of the platelets to the exposed subendothelial structures takes place.

Transport of blood components, for example platelets, to a site of hemostatic plug or thrombus formation, is determined by their distribution and by fluid dynamic variables, e.g. flow velocity and the distribution of velocity gradients or shear rates in a vessel. The science dealing with the flow properties of blood is called blood rheology or hemorheology (Copley and Seaman, 1981). Initially, blood was studied as a bulk fluid (macrorheology). Macrorheology is relevant to understand the flow behavior of blood in large vessels (macrocirculation). When the technology improved, the study of the flow behavior of individual blood cells (microrheology) became more and more important. However, it should be emphasized that the flow behavior of blood in large vessels differs in many aspects from that in small vessels (microcirculation). To understand the latter, knowledge of the flow behavior of individual cells (microrheology) is important.

\subsection{GENERAL FLUID DYNAMICS}

Flow of a simple, Newtonian fluid in a tube is laminar if velocities are not too high. An example of such a fluid is water. If we consider layers of a non-pulsatile fluid from the wall towards the tube center, first a thin layer 
of fluid is encountered at the wall which remains stationary (no-slip condition). In each successive layer of fluid the velocity is higher, till in the tube center the velocity is found to be maximal. Laminar flow of a Newtonian fluid (see paragraph on viscosity of a fluid) is described by Poiseuille's law.

\section{Poiseuille's law}

Poiseuille (1843) derived empirically, under laminar flow conditions, the relationship between flow rate, the pressure difference over a tube, its length and radius, and the viscosity of a fluid:

$$
Q=\pi \frac{\Delta p}{1} \cdot \frac{R^{4}}{8 \eta}
$$

where $\mathrm{Q}=$ the flow rate (volume/unit time), $\Delta \mathrm{p}=$ pressure difference over the tube, $R=$ inner radius of the tube, $\mathbb{l}=$ length of the tube and $\eta$ $=$ viscosity of the fluid. This equation is only valid for laminar, non-pulsatile flow of a Newtonian fluid. In the circulation, however, blood flow is pulsatile. In addition, blood is a non-homogeneous fluid and the geometry of blood vessels is complex. Therefore, this equation should be interpreted with caution when dealing with the flow of blood in blood vessels in vivo. The velocity profile for a Newtonian fluid in the absence of pulsatility has the shape of a parabola.

\section{Viscosity of a fluid}

One important factor in the Poiseuille equation that is not related to the tube, but to the fluid itself is the viscosity. Resistance to flow of the fluid is proportional to its viscosity. The viscosity of a fluid is determined by the internal friction between adjacent fluid layers. In Figure 2.1 two adjacent layers of fluid are depicted schematically. The relative movement of adjacent layers is determined by the force $(F)$ per unit area $(A)$ between the layers $(\mathrm{F} / \mathrm{A})$, which is called shear stress $(\tau)$. The unit for shear stress is Pascal (Pa) and is usually expresses in the form of milli-pascal $(\mathrm{mPa})$ when blood is used. The relative movement of adjacent layers results in a velocity gradient across the vessels. This velocity gradient $(\mathrm{dv} / \mathrm{dx})$, or shear rate $(\gamma)$ is expressed in $\mathrm{s}^{-1}$. 
The mean shear rate in a tube with flow obeying Poiseuille's law is given by:

$$
\text { Mean shear rate }=\frac{4 \text { xmean velocity }(\mathrm{m} / \mathrm{s})}{\text { tube radius }(\mathrm{m})}
$$

Mean shear rate increases with flow velocity. A decrease in vessel diameter condenses the flow streamlines, and, hence, increases the mean shear rate.

The viscosity of a fluid is defined as the ratio between shear stress and shear rate; it is expressed in mPa.s:

$$
\operatorname{Viscosity}(\eta)=\frac{\text { Shear stress }(\tau)}{\text { Shear rate }(\gamma)}
$$

Fluids with a constant viscosity at all levels of shear rate are called Newtonian. If viscosity changes with shear rate the fluid is called nonNewtonian. Blood plasma and physiological saline are examples of Newtonian fluids. Blood is a non-Newtonian fluid, due to the presence of many red blood cells. Blood with a hematocrit less than $30 \%$ displays nearly Newtonian behavior (Merril, 1969). Viscosity is usually determined in a viscometer. A sample of the fluid is subjected to a uniform level of shear rate, and the shear stress is measured. In case of a Newtonian fluid only one measurement is required. For a non-Newtonian fluid measurements over the whole range of expected shear rates is required.

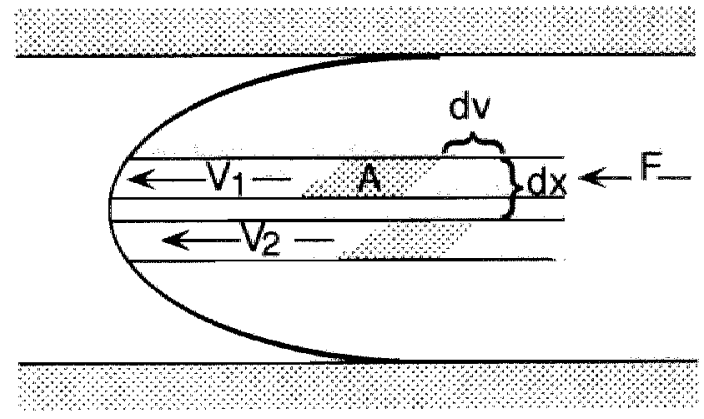

Figure 2.1: Longitudinal section of a cylindrical tube showing a schematic presentation of the concept of shear. 
When a fluid is flowing through a tube, shear rate varies from wall to center. In case of a Newtonian fluid, viscosity could be determined using Poiseuille's law. Blood is a non-Newtonian fluid, since shear stress and shear rate are not linearly related. If one nevertheless calculates the viscosity of blood from Poiseuile's law, the viscosity coefficient is called apparent viscosity. The apparent viscosity of blood differs greatly between small and large vessels.

\section{Reynolds number}

In almost all parts of the circulation blood flow is laminar, i.e. layers of blood do not cross each other. By contrast, in turbulent flow blood is moving across the vessel, leading to random movements of the blood cells. The critical point at which flow changes from laminar to turbulent is described by the Reynolds number (Re), which represents the ratio of inertial to viscous forces:

$$
R e=\frac{p \cdot \bar{V} \cdot d}{\eta}
$$

where $\rho$ is fluid density, $\bar{V}$ is mean flow velocity, $\eta$ is the fluid viscosity and $d$ is the vessel diameter. In the circulation of a resting human being, Reynolds numbers are estimated to vary from about $10^{-3}$ in capillaries to peak values as high as $10^{3}$ in the aorta (Karino and Goldsmith, 1987). In normal physiological situations, turbulence may probably only occur in the ascending aorta. The Reynolds number becomes far less than unity in arterioles, capillaries and venules. At such small Reynolds numbers viscous forces become predominant, while the inertial forces are negligibly small. Because of the dominance of viscous forces, velocity profiles of Newtonian fluids will have reached their final shape in microvessels within a distance of about one vessel diameter. In case of blood, the exact entrance length is not known.

\subsection{WHOLE BLOOD VISCOSITY}

In blood vessels with diameters larger than about $500 \mu \mathrm{m}$, the size of the blood cells is small enough as compared to vessel diameter to neglect the particulate nature of the blood, approximating it as a homogeneous fluid. 


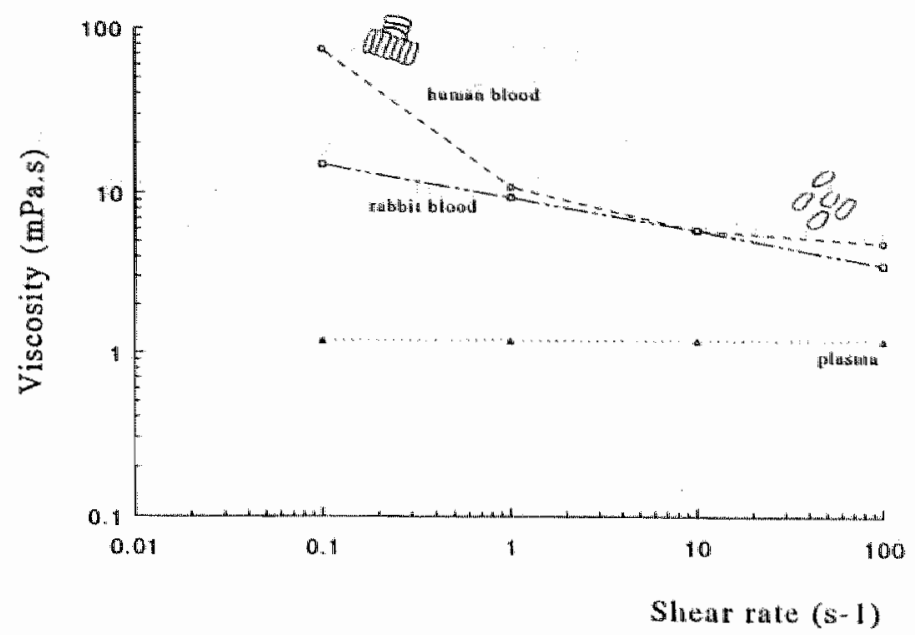

Figure 2.2: Logarithmic relation between apparent viscosity and shear rate for Newtonian (plasma) and non-Newtonian (rabbit and human blood) fluids. Insets show red blood cell aggregation at low shear rates and red blood cell disaggregation and deformation at high shear rates.

As mentioned before, initially, rheological studies focused mainly on the bulk parameter of whole blood viscosity.

Whole blood viscosity depends on hematocrit, red blood cell aggregation, red blood cell deformability and plasma viscosity. These factors are not independent of one another. As is shown in Figure 2.2, the viscosity of whole human blood shows non-linear behavior, in contrast to the viscosity of Newtonian fluids, which displays a linear relationship with shear rate. Figure 2.2 also shows that the viscosity of whole rabbit blood rises less with decreasing shear rate than human blood (unpublished result). Blood of sheep and goat shows the same tendency as rabbit blood, albeit more pronounced (Chien et al, 1971). The shear rate dependency of whole blood viscosity is caused by red cell aggregation and red cell deformation. At high shear rates, the viscosity of blood depends primarily on the deformability or fluidity of the red blood cells, while at low shear rates aggregation of red blood cells is the most important determining factor.

\section{Hematocrit}

Red blood cells occupy the largest volume fraction of the blood cells and, hence, their concentration or hematocrit is an important determinant of whole blood viscosity (Chien et al, 1984). An almost linear relationship exists between hematocrit and whole blood viscosity up to an hematocrit 
value of about $60 \%$. At higher hematocrit values the relationship becomes exponential.

At low shear rates an increase in hematocrit may have a dramatic effect on bloud viscosity, whereas this influence is less at high shear rates (Wells et al, 1961). Because it is assumed that in veins shear rates are relatively low, changes in hematocrit may strongly influence whole blood viscosity and, hence, resistance to flow in these vessels (Driessen et al, 1979; Kiesewetter et al, 1979).

\section{Red blood cell aggregation}

Fahraeus (1929) observed that normal red cells in plasma form linear aggregates under low shear conditions. These linear red blood cell aggregates called rouleaux are formed in the presence of fibrinogen and other macromolecules (Chien and Jan, 1973; Chien et al, 1983). These reversible red blood cell aggregates disturb the flow streamlines and cause an increase in whole blood viscosity.

Red blood cell aggregation occurs when the macromolecular bridges formed between red blood cells are strong enough to withstand the disaggregating energies due to electrostatic repulsion between the negatively charged red blood cells (Chien and Jan, 1973). Red blood cells treated with neuraminidase showed an increase in red blood cell aggregation. Neuraminidase destroys $\mathrm{N}$-acetyl-neuraminic acid molecules on the red blood cell membrane, which are mainly responsible for the negative charge of the red blood cells (Jan and Chien, 1973).

\section{Red blood cell deformability}

Red blood cells are able to fold their membrane into a kaleidoscope of shapes without fragmentation. This flexibility of the red blood cell membrane is referred to as red blood cell deformability. The absence of a nucleus, the low cytoplasmic viscosity, the viscoelasticity of the membrane and the high ratio of surface area to cell volume, all contribute to a high deformability. A decrease in red blood cell deformability may be caused by changes in the erythrocyte membrane, for example caused by acidosis or by variations in red blood cell geometry due to alterations in hemoglobin as in sickle cell anemia (Chien et al, 1970a; Murphy, 1966), or hereditary spherocytosis (Murphy, 1966). Experiments performed by Pfafferott and coworkers (1985) showed that the difference in deformability between young and old red blood cells depends on changes in cytoplasmic viscosity, and less 
on differences in membrane viscoelasticity.

Resistance to bulk flow of blood is reduced considerably by the deformability of red blood cells, since they can adapt an ellipsoid shape and rotate their membrane around the interior of the cell (Schmid-Schönbein and Wells, 1969). Due to red cell deformability, apparent blood viscosity is relatively low in the majority of the larger blood vessels under normal circulatory conditions. Glutaraldehyde treated red blood cells are less deformable (Chien et al, 1970b) and increase whole blood viscosity at various shear rates.

Most experiments on red cell deformability have been performed with human cells. Sheep and goat red blood cells have been suggested to be less deformed than those of other mammalian species. Especially at higher shear rates the viscosity, which depends in this region primarily on the deformability, was more increased in goat and sheep blood than in human blood. This is probably due to reduced viscoelasticity of the red blood cell membrane (Chien et al, 1971). A low degree of membrane flexibility may be caused by a low ratio of surface area to cell volume and/or a high membrane rigidity (Chien et al, 1970a). The more deformable a red blood cell, the narrower and longer it can become. In Figure 2.3 the elongation index (see chapter 3), an indicator of deformability, is shown for rabbit and human red blood cells.

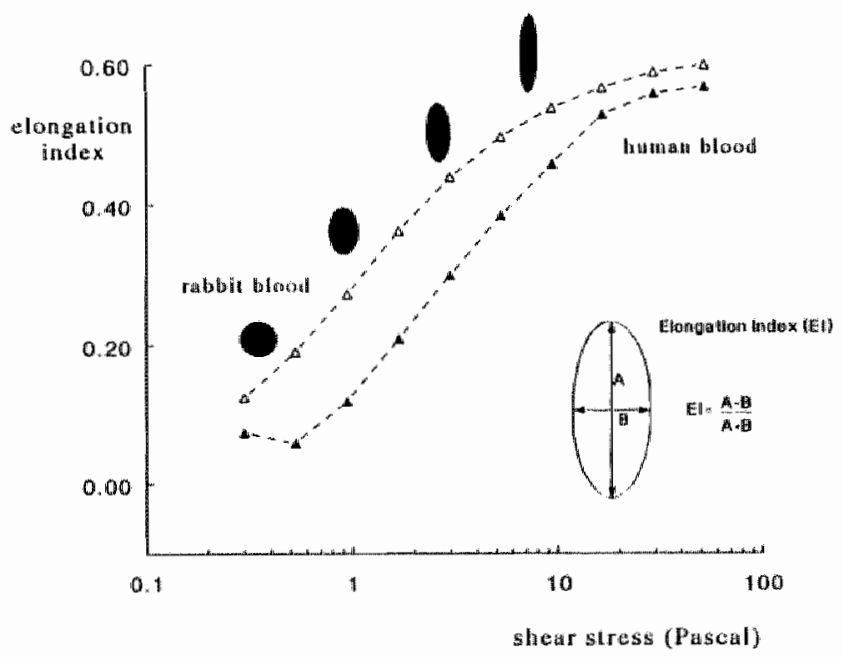

Figure 2.3: Difference between deformability of human and rabbit red blood cells. Diffraction patterns of human red blood cells are shown also. With permission of Dr MR Hardeman, Dept. of Internal Medicine and Clinical Chemistry, Academic Medical Centre, Amsterdam, the Netherlands. 
Human red blood cells are less deformable, especially at low shear values as compared to rabbit red blood cells (unpublished result). This is in agreement with the observed lower viscosity of rabbit blood as compared to human blood (see Figure 2.2).

\section{Plasma viscosity}

Plasma viscosity is mainly determined by the plasma proteins and temperature. Plasma is a suspension of proteins and smaller molecules in water, with Newtonian characteristics, i.e. viscosity is independent of shear rate. The suspended molecules disturb the fluid streamlines during flow. This requires increased dissipation of energy, and thus increased viscosity. In normal human plasma albumin contributes for only $36 \%$ to plasma viscosity, although it comprises $60 \%$ of the total protein weight (Mayer et al, 1966). By contrast, fibrinogen, accounting only for about $4 \%$ of the plasma protein weight, has a more pronounced effect on plasma viscosity. Its influence on plasma viscosity can be seen in the difference between plasma and serum viscosity: the viscosity being $20 \%$ less in serum than in plasma. This large effect of fibrinogen on plasma viscosity is due to its high molecular weight (mol wt 340.000) and elongated shape. An increase in plasma viscosity can clinically be observed in the presence of paraproteins (Lowe, 1988).

\subsection{MICROCIRCULATION}

The concept of whole blood viscosity can only be applied to larger vessels (see subsection 2.2). In vessels smaller than about $100 \mu \mathrm{m}$ (microcirculation), the flow behavior of individual blood cells becomes increasingly important (microrheology). In these vessels Reynolds numbers are less than unity.

The vessels of the microcirculation consist of arterioles, capillaries and venules. Arterioles feed the capillaries and possess a wall with one or two layers of smooth muscle cells (Rhodin, 1967). On the contrary, the wall of the draining postcapillary venules does not have a confluent layer of smooth muscle cells, but possesses a discontinuous layer of pericytes or primitive smooth muscle cells (Rhodin, 1968). Beside these morphological differences between arterioles and venules, other rheological and functional differences have to be appreciated. In arterioles flow diverges at branch points, while in venules it converges. Arterioles and venules also differ in permeability 
(De Clerck et al, 1985) and in their sensitivity to monoamines (Heltianu et al, 1982). In addition, the thromboembolic reaction following wall puncture is more pronounced in arterioles than in venules (oude Egbrink et al, 1988; 1989). Even in vessels of the same type but of different sizes, e.g. between rabbit aortas and umbilical arteries, differences in thrombogenic properties as related to platelet function can be found (Escolar et al, 1991). In venules marginating leukocytes may be observed, which are usually not present in arterioles (see paragraph on leukocytes in microvessels).

In the microcirculation, blood flow conditions differ considerably from those in the macrocirculation. How fast a velocity profile is restored after it has been disturbed at a bifurcation, depends on the Reynolds number of flow. In the macrocirculation the Reynolds numbers are relatively high. The entrance length, defined as the distance of transition from disturbed inflow to a section where the velocity differs from the fully developed profile by less than $1 \%$, is so long that the profiles never completely develop. At very low Reynolds numbers, a condition generally present in the microcirculation, the entrance length is $1.3 \times R$, where $R$ is the vessel radius. Consequently, in the microcirculation the entrance length is less than one vascular diameter (2R); the flow becomes fully developed, albeit not parabolic, over most of the length of the microvessel (Slaaf et al, in press). In the macrocirculation the profiles are flattened due to the pulsatility and the long entrance length, in the microcirculation the profiles are flattened because of the nonNewtonian character of the blood under these conditions.

\section{Fåhraeus-Lindqvist effect: behavior of red blood cells}

The importance of microrheological parameters in vessels of the microcirculation is indicated by the Fåhraeus-Lindqvist effect (Fåhraeus and Lindqvist, 1931). This effect is defined as the decline in apparent blood viscosity with decrease in vessel diameter (Chien, 1977). This decline progresses until the tube diameter reaches a value of about $5-7 \mu \mathrm{m}$ (Gaehtgens, 1980). In rapidly perfused capillaries this effect can be so pronounced that apparent blood viscosity almost equals plasma viscosity (Zilow and Linderkamp, 1989). In capillaries with smaller diameters, the trend of this effect becomes reversed (Chien et al, 1984).

An important determinant of this Făhraeus-Lindqvist effect is the decrease in local hematocrit with decreasing vessel diameter (Gaehtgens, 1980). This decrease in local hematocrit is designated the Fåhraeus effect. In microvessels red blood cells tend to migrate towards the vessel center. This provokes a higher velocity of the cells relative to that of plasma. 
Statistically red blood cells pass a microvessel more rapidly than plasma, and hence, the fraction of red cells in the vessel will be lower than that of plasma. This effect becomes especially important in microvessels smaller than $100 \mu \mathrm{m}$.

In capillaries the Fahraeus-Lindqvist effect is most pronounced due to a relatively low hematocrit. In addition, capillary hematocrit is known to be influenced by the composition of the endothelial glycocalyx (Desjardins and Duling, 1990). Moreover, to be able to pass through narrow capillaries whose diameter is often smaller than that of the red blood cells, these cells must maintain a highly deformable state (Braasch, 1971). The red blood cells probably deform into elastically-walled cups in these narrow vessels (Whitmore and Stalker, 1976). If the red blood cells lose their deformable character, blood flow through the microcirculation may be impeded (Replogle et al, 1967). Glutaraldehyde treated red blood cells are less deformable (Chien et $\mathrm{al}, 1970 \mathrm{~b}$ ) and cause a decrease in flow in several microcirculatory beds in the rat (Simchon et al, 1987).

\section{Platelet rheology in microvessels}

The knowledge of platelet behavior in flowing blood was till recently almost exclusively based on the findings in theoretical models and in vitro experiments, the latter employing rigid spheres and disks. On this basis an inward radial migration was predicted for the flexible red blood cells flowing in small blood vessels (Goldsmith, 1971, 1973). By contrast, a tendency to inward migration of the blood platelets, if any, would be considerably smaller (Jeffery, 1922; Segre and Silberberg, 1962; Chaffey et al,1965; Goldsmith and Mason, 1967; Karnis and Mason, 1967; Goldsmith, 1972). In vitro, inward migration of individual red blood cells results in an increase in local hematocrit in the tube center (Palmer, 1965; Goldsmith, 1967; Palmer and Betts, 1975). Because of this central red cell crowding, it was assumed that the lighter platelets would be dispersed from the center of the vessel, resulting in a higher platelet concentration near the wall. Experiments with blood suspensions flowing through glass channels (Eckstein et al, 1988; Tilles and Eckstein, 1987) or slits (Palmer, 1967) indeed showed a higher platelet concentration near the wall. Moreover, studies in perfusion chambers have shown that platelet deposition on subendothelium increases with hematocrit (Turitto and Baumgartner, 1975; Turitto and Weiss, 1980). An increase in red blood cell size (Aarts et al, 1983) and a decrease in red blood cell deformability (Aarts et al, 1984) both enhance platelet transport towards the wall of the perfusion chamber. 
The first studies on the flow behavior of individual platelets in vivo have been performed in capillaries which contained a few red blood cells, and in larger vessels using slowly flowing or stagnant plasma (Bizzozzero, 1882; Silver and Stehbens, 1965; Kwaan et al, 1976). However, in arterioles and venules with undisturbed flow, platelets could only be observed if they were located close to the endothelial wall (Bloch, 1962; Schmid-Schönbein and Zweifach, 1975). Recently, individual platelets could be visualized flowing in a microvessel amidst the normal excess of red blood cells (Tangelder et al, 1982a). To this end the platelets were labelled in vivo with a fluorescent dye and observed with fluorescence microscopy. Using this methodology in rabbit mesenteric arterioles, it was shown that platelet concentration near the wall was about twice the concentration in the center of the vessel (Tangelder et al, 1985). This finding was similar to the findings obtained in vitro (see above). So far, no such experiments have been performed in venules. Despite the similarity between the findings on platelet distribution in arterioles and in vitro, such studies in venules are needed, because platelets quickly respond to slight changes in their environment and venules differ from arterioles in many aspects (see section 2.3).

When blood vessels are traumatized, platelets make contact with the injured vessel wall and with each other, forming a thrombus (Tangelder et al, 1989). Under normal circumstances circulating platelets also come frequently in apparent contact with the vessel wall (Tangelder et al, 1985, 1986), but do not stick to the endothelium. In arterioles, platelets tend to align with flow (Teirlinck et al, 1984), but they can be seen tumbling especially near the vessel wall (Tangelder et al, 1988). The tendency to align with flow is strongest near the wall. Towards the center of the arteriole their orientation is more random. Since shear rate increases from the center of the vessel towards the wall, this finding implies that in arterioles the tendency of the platelets to align with flow increases with shear rate.

Wall shear rate or velocity gradient is an important factor in describing interactions between blood cells, plasma components and the vessel wall (Goldsmith and Turitto, 1986; Turitto, 1982). Most of the information available about velocity profiles in microvessels has been obtained in vitro. These in vitro studies have yielded conflicting results and have not been performed in glass tubes smaller than $30 \mu \mathrm{m}$ (Bugliarello and Hayden, 1963; Bugliarello and Sevilla, 1970; Goldsmith, 1972; Baker and Wayland, 1974; Goldsmith and Marlow, 1979). Experiments performed by Tangelder and co-workers, (1986) showed that in rabbit mesenteric arterioles the velocity profilles of blood platelets differ significantly from a parabolic velocity distribution. The profiles are flattened as compared to a 
parabola. As a consequence of the blunted velocity profile in arterioles, wall sheat rates in these vessels are on the average two times higher than expected on the basis of a parabolic velocity profile with the same volume llow (Tangelder et al, 1988). Because in mesenteric arterioles platelet concentration near the wall is about twice the concentration in the center (Tangelder et $\mathrm{al}_{*}, 1985$ ) approximately $70 \%$ of the platelets are flowing within the high shear domain near the vessel wall. In case of a wall injury in microvessels, a relatively high percentage of platelets with a relatively low velocity will come in contact with activating agents released from the damaged vessel wall or from activated platelets themselves. Puncture of the wall of arterioles and venules in vivo induces in these microvessels the rapid formation of a thrombus similar in height, but with a number of emboli produced which is 6 times higher in arterioles than in venules (oude Egbrink et al,1988). This thrombus forms a stenosis which influences local flow velocity and wall shear rate. No relation could be found between the absolute level of wall shear rate in a stenosed segment, which may be as high as $16,000 \mathrm{~s}^{-1_{1}}$ and the number of emboli produced (oude Egbrink et al, 1991b). The stop of platelet aggregation under such high shear conditions is not in agreement with in vitro data, which suggest a positive relation between platelet aggregation and wall shear rates up to a value of $10,000 \mathrm{~s}^{-1}$ (Turitto and Baumgartner, 1979; Badimon et al, 1987). This discrepancy might be explained by the production of platelet inhibitors by the vessel wall, e.g. nitric oxide and prostacyclin $\left(\mathrm{PGI}_{2}\right)$.

\section{Leukocytes in microvessels}

Leukocytes occupy only a small percentage of whole blood volume. Consequently, in large vessels they have little influence on the flow behavior of normal blood. In small vessels, however, leukocytes have been shown to contribute to apparent blood viscosity (Schmid-Schönbein et al, 1980a). Leukocytes are less deformable than red blood cells (Chien, 1988). Moreover, in solution they have a spherical shape.

Leukocytes exhibit a nonuniform distribution over the cross-section of a small tube, as indicated by in vitro experiments. This behavior of leukocytes depends on the rheological conditions of red blood cells. When red blood cells are dispersed, the larger leukocytes move to the center of the stream (Palmer, 1967; Nobis et al, 1985) and exhibit a Făhraeus effect similar to red blood cells (Chien et al, 1984). However, when red blood cells are aggregated, leukocytes are dispersed to the vessel wall region (Paimer, 1967; Nobis et al, 1985; Goldsmith and Spain, 1984). 
In mesentery exposed for intravital microscopy, leukocytes roll along the wall of venules at a velocity clearly lower than that of free flowing blood cells (Atherton and Born, 1972; Schmid- Schönbein et al, 1980b; House and Lipowsky, 1987; Tangelder et al, 1991; oude Egbrink et al, 1992). This behavior which is usually not observed in arterioles, also points to a possible functional difference between arteriolar and venular endothelium. Adherence of leukocytes to venular endothelium may cause an increased resistance to flow, due to a reduction of the effective luminal diameter by the adhering leukocytes (Lipowsky et al, 1980). This may be especially important in low flow states, e.g. during shock (Bagge et al, 1980)

\section{Interactions in microvessels between blood cells}

Leukocytes rolling in venules along the wall (see paragraph on leukocytes in microvessels) and blood platelets which are flowing near the vessel wall may influence each other. In rabbit mesenteric venules the thromboembolic reaction occurring after vessel wall injury, is known to cause a decrease in the number of rolling leukocytes (oude Egbrink et al, 1992). Platelets observed in blood vessels of the rabbit ear chamber adhere to leukocytes which had stuck to the endothelial wall or rolled along it (Silver and Stehbens, 1964). These authors reported that platelets never roll along endothelium and only rarely adhered to the intact vessel wall. However, in our own experiments we have observed that occasionally platelets do roll in venules.

Leukocytes also influence the flow of red blood cells, especially in capillaries. In glass tubes with diameters close to the leukocyte diameter, leukocytes generally travel slowly. These leukocytes create an empty plasma column in front and an accumulation of red blood cells behind them (Gaehtgens, 1980; Schmid-Schönbein et al, 1980a). This train of red blood cells which follow the slowly moving leukocyte is densely packed. This heterogeneous distribution of cells and plasma then gives rise to considerable fluctuations in apparent blood viscosity.

As has been pointed out in the paragraph on platelet rheology in microvessels, red blood cells have a distinct influence on platelet distribution. Central crowding of the red blood cells will expel the lighter platelets from the center towards the vessell wall. In vivo, the platelet concentration near the wall of arterioles was indeed found to be twice the central concentration (Tangelder et al, 1985). 


\subsection{DEXTRANS AND BLOOD RHEOLOGY}

Dextrans are polymers of D-glucose and are synthesized by certain bacteria (Leuconostoc mesenteroides). These bacterial dextrans, with a mollecular weight of about two million, can be changed into smaller fragments by partial acid hydrolysis. Dextrans as such are electrically neutral, but can be charged positively (e.g. diethylaminoethyl dextran) or negatively (e.g. dextran sulfate) charged. The structure of a neutral dextran molecule is shown in Figure 2.4. Dextrans have been used in clinical practice for more than 20 years, mainly as a plasma substitute to improve blood flow. Their ability to reverse aggregation of red blood cells in whole blood. Low molecular weight dextran (molecular weight 40.000) has indeed disaggregating effects on red blood cells in humans and in several animals (Engeset et al, 1967). Gelin and Ingelman (1961) postulated that this disaggregation was a direct effect of low molecular weight dextran on red blood cells, while Meiselman and coworkers (1967) proposed that this disaggregating effect is caused by volume dilution. Microcirculatory studies performed in the ear chamber of rabbits showed that counteraction of induced intravascular red blood cell aggregation by low molecular weight dextran results from improved blood flow rather than disaggregation (Engeset et al, 1967). Later experiments performed by Jan et al, (1982) suggest that this disaggregating effect is primarily due to an elevation of surface potential of the red blood cells.

In contrast to low molecular weight dextran, high molecular weight dextrans (mol wt $>150.000$ ) can cause red cell aggregation (Thorsen and Hint, 1950), producing rouleaux which are morphologically similar to those generated by fibrinogen.

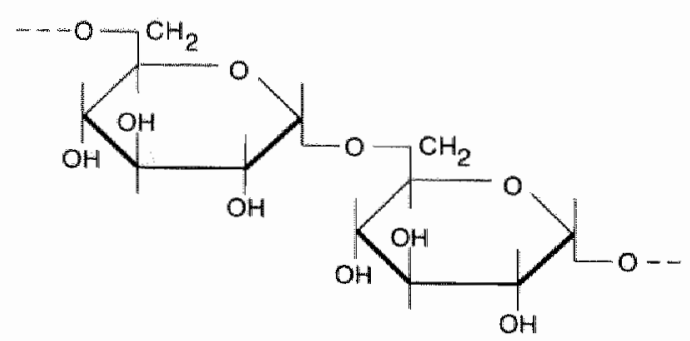

Figure 2.4: Schematic representation of a part of the molecular structure of a neutral dextran. 
Dextran binds to the red blood cell surface via nonspecific adsorption (Brooks, 1973) and induces a shape transformation from normal discocytes to cupped cells, which leads to an alteration in red blood cell deformability (Nash and Meiselman, 1983).

\section{The influence of dextrans on red blood cells}

As mentioned above, dextrans can induce red blood cell aggregation. The induction of aggregates increases with increasing molecular weight of the dextran (Chien and Jan, 1973). This can be explained by the bridging theory, i.e. the ends of the larger aggregating molecules adhere to adjacent cells, cross-linking the cells over such a distance that the total adhesive force is greater than that of repulsion. However, below a critical distance the repulsive forces are too great for the small dextrans to overcome. When the negative charge from the red blood cell surface is reduced by neuraminidase treatment, which removes sialic acid (Jan and Chien, 1973), then even low molecular weight dextrans (mol wt $<70.000$ ) are able to aggregate red blood cells.

Dextrans adsorb to red blood cell membranes very weakly (Chien, 1980) and it is likely that they are bound through Van der Waal's forces. Although dextrans bind to the membrane phospholipids, it is its adsorption to glycoproteins or glycolipids that is probably responsible for aggregation (Brooks, 1973). Nevertheless, the similarity in the binding isotherms for dextran to normal cells and those treated with neuraminidase implies that sialic acid is not involved in the adsorption (Chien et al, 1977).

\section{Dextrans and blood platelets}

Dextrans bind to the surface of platelet membranes (Bygdeman and Eliasson, 1967; Langdell et al, 1958; Maupin, 1969) and probably influence their negative surface charge. This negative surface charge of platelets under physiological circumstances has been suggested to be important for platelet adhesion and aggregation. At low doses dextran sulfate is known to induce a decrease in electrophoretic mobility of platelets, probably by formation of a dextran- $\mathrm{Ca}^{2+}$ or $\mathrm{Mg}^{2+}$ complex on the platelet surface producing a decrease in negative charge (Stoltz, 1983). High doses of dextran sulfate, however, show an increase in platelet electrophoretic mobility. Platelet aggregation is to all likelihood not induced by binding of dextrans to platelets.

Dextrans are known to interfere with the mechanism of thrombosis in 
several ways. A prolonged bleeding time has been observed after dextran injections (Bygdeman and Eliasson, 1967; Adelson et al, 1955). Abnormal bleeding was not only related to the amount injected, but also to the molecular weight of the dextran (Bromwell et al, 1954; Howard, 1956; Langdell et al, 1958). High molecular weight dextran produces a more severe prolongation of the bleeding time than low molecular weight dextran. Experiments performed in rabbit arterioles by Arfors and Bergqvist (1975, 1976) showed that a neutral $40 \mathrm{kD}$ or a $70 \mathrm{kD}$ dextran, injected into the marginal ear vein of the rabbit in a dose of $1.0 \mathrm{~g} / \mathrm{kg}$ bodyweight, had no effect on haemostatic plug formation time.

In vitro experiments, using human blood, showed that high molecular weight dextrans diminish prothrombin production, which leads to reduced thrombin formation (Jacobaeus, 1957). The production of prothrombin and the antithrombin III activity is increased in the presence of low molecular weight dextran. Intravenous infusion of low molecular weight dextrans causes a decrease in adenosine-diphosphate (ADP) induced adhesion and aggregation of human platelets (Bygdeman and Eliasson, 1967). In rabbits, injection of a low molecular weight sulfated dextran in a dose of $60 \mathrm{mg} / \mathrm{kg}$ shows an inhibited thrombin and collagen induced platelet aggregation until 60 minutes after administration (Nakajima et al, 1978). In addition, no influence was found on the aggregation rate of ADP induced platelet aggregation (Nakajima et al, 1978).

\section{Dextran sulfate and leukocytes}

Dextran sulfate, a negatively charged derivative of dextran, is known to reduce the number of rolling leukocytes in vivo in venules in a dosedependent way (Tangelder et al, 1991). This inhibition is independent of molecular weight, but is influenced by the average number of sulfate groups per monosaccharide. Dextran with a sulfate content of $17 \%$, i.e. an average of 1.9 sulfate residues per monosaccharide, given in a dose of $20-23 \mathrm{mg} / \mathrm{kg}$ bodyweight, shows a $90 \%$ inhibition of leukocyte rolling for a duration of about 2 hours . 


\section{METHODOLOGICAL SURVEY}

In this chapter a brief outline of the most important aspects of the materials and methods used in the studies described in this thesis is presented. For information about practical details the reader is referred to the various chapters.

\section{Rabbits}

All experiments were performed on approximately 3 months old New Zealand white rabbits of either sex, ranging in weight from 1.9 to $3.9 \mathrm{~kg}$. The rabbits were supplied by a commercial breeder (Iffa Credo/Broekman BV, Someren, the Netherlands). Until the experiments started, they had access ad libitum to water and food (Rabbit Diet LKK-20, Hope Farms BV, Woerden, the Netherlands). The drinking water contained $40 \mathrm{mg}$ methoxasole-T-trimethoprim and $200 \mathrm{mg}$ sulfamethoxasole per litre (A.U.V. Cuyk, the Netherlands). The ears of the rabbits were treated with linimentum lindani (FNA, the Netherlands) to prevent them against scabies.

Anesthesia was induced by intramuscular injections of ketamine and xylazine and maintained by a continuous intravenous infusion of the same compounds. This combination provides adequate muscle relaxation and analgesia, but it can cause cardiovascular and respiratory depression. Especially, xylazine is known to reduce blood pressure by decreasing cardiac output and depressing the central vasomotor center (Popilskis, 1991). In Table 3.1A the values of the cardiovascular and respiratory variables as obtained in the spontaneously breathing anesthetized rabbits used in this thesis and those found in the literature are compared.

For intravenous infusion the anesthetics were dissolved in a lactetrol solution (see Table 3.3) and infused at a rate of $15 \mathrm{ml} /$ hour. This lactetrol solution prevents the animal from dehydration and metabolic acidosis and maintains the electrolyte balance in the plasma at normal levels. A catheter was inserted into the femoral artery to measure arterial blood pressure. Through this catheter $5 \mathrm{ml}$ physiological saline were infused per hour to prevent clotting at the tip of the catheter. Heart rate was assessed from the 
Table 3. LA: Cardiovascular and respiratory variables under anesthesia with ketamine and xylazine in spontaneously breathing New Zealand white rabbits: mean arterial blood pressure $(M A P ; m m H g)$, heart rate $\left(\mathrm{HR} ;\right.$ beats/min) and arterial $\mathrm{pH}, \mathrm{pO}_{2}(\mathrm{mmHg})$ and $\mathrm{pCO}_{2}$ $(\mathrm{mmHg})$. Mean value and standard deviation, if available, are given.

SPONTANEOUSLY BREATHING RABBITS

\begin{tabular}{lllllll}
\hline & $\mathrm{n}$ & $\mathrm{MAP}$ & $\mathrm{HR}$ & $\mathrm{pH}$ & $\mathrm{pO}_{2}$ & $\mathrm{pCO}_{2}$ \\
\hline $\begin{array}{c}\text { oude Egbrink } \\
\begin{array}{c}(1988) \\
\text { Peeters }\end{array}\end{array}$ & 15 & $69 \pm 14$ & $133 \pm 24$ & $7.34 \pm 0.03$ & $48 \pm 5$ & $51 \pm 4$ \\
$\begin{array}{c}(1988) \\
\begin{array}{c}\text { Popilskis } \\
\text { (1991) }\end{array}\end{array}$ & 6 & 53 & 229 & 7.43 & 63 & 42 \\
$\begin{array}{c}\text { Woldhuiss } \\
\text { (thesis) }\end{array}$ & 6 & $60 \pm 11$ & $184 \pm 18$ & $7.35 \pm 0.2$ & $69 \pm 4$ & $43 \pm 4$ \\
\hline
\end{tabular}

${ }^{1}$ personal communication. Rabbits were Flemish Giants and not New Zealand White. dose intramuscularly: ${ }^{2} 50 \mathrm{mg} / \mathrm{kg}$ ketamine and $5 \mathrm{mg} / \mathrm{kg}$ xylazine, ${ }^{3} 35 \mathrm{mg} / \mathrm{kg}$ ketamine and $5 \mathrm{mg} / \mathrm{kg}$ xylazine, ${ }^{4} 40 \mathrm{mg} / \mathrm{kg}$ ketamine and $4 \mathrm{mg} / \mathrm{kg}$ xylazine, prior to ventilation.

Table 3.1B: Cardiovascular and respiratory variables in artificially ventilated anesthetized New Zealand white rabbits: mean arterial blood pressure (MAP; $\mathrm{mmHg}$ ), heart rate (HR; beats/min) and arterial $\mathrm{pH}, \mathrm{pO}_{2}(\mathrm{mmHg})$ and $\mathrm{pCO}_{2}(\mathrm{mmHg})$. Mean values and standard deviation, if available, are given.

\section{ARTIFICIALLY VENTILATED RABBITS}

\begin{tabular}{lcccccc}
\hline & n & MAP & HR & pH & $p_{2}$ & $\mathrm{pCO}_{2}$ \\
\hline & & & & & & \\
oude Egbrink, 1988 & 13 & $65 \pm 8$ & $119 \pm 18$ & $7.40 \pm 0.03$ & $105 \pm 13$ & $33 \pm 6$ \\
oude Egbrink, 1991 & - & $74 \pm 5$ & $92 \pm 12$ & $7.45 \pm 0.04$ & $96 \pm 8$ & $43 \pm 2$ \\
oude Egbrink, 1992 & 34 & $65 \pm 8$ & $118 \pm 21$ & $7.42 \pm 0.03$ & $99 \pm 13$ & $38 \pm 6$ \\
Woldhuis, thesis & 46 & $74 \pm 9$ & $139 \pm 18$ & $7.46 \pm 0.06$ & $89 \pm 13$ & $37 \pm 6$
\end{tabular}

Anesthesia was maintained during artificial ventilation by IV infusion of $40 \mathrm{mg} / \mathrm{kg} / \mathrm{hour}$
ketamine and $5 \mathrm{mg} / \mathrm{kg} /$ hour xylazine. 
femoral arterial pressure pulse.

To prevent respiratory depression induced by the anesthetics, animals were ventilated with a mixture of air and oxygen through a tracheal cannula connected to an infant ventilator. The respiratory rate was set at $60 / \mathrm{min}$. Total gas flow through the ventilator was set at $2.51 / \mathrm{min}$, and the inspiration to expiration ratio at $1: 1.5$. This resulted in a respiratory minute volume of 1.01 and, hence, a tidal volume of about $17 \mathrm{ml}$, which is normal for these rabbits (Kozma, 1974). Blood gas analyses were performed 10-15 minutes after the start of ventilation in blood collected from a central ear artery (see Table $3.1 \mathrm{~A}$ and $3.1 \mathrm{~B}$ ) and examined every 30 minutes. Table 3.1B shows the mean values of the cardiovascular and respiratory parameters during artificial ventilation. The blood gas values varied about the mean during the experimental period of about 2 hours. Comparison of Tables 3.1A and 3.1B (oude Egbrink et al, 1988; Woldhuis et al, thesis) shows that ventilation did not change mean arterial blood pressure or heart rate. In awake adult rabbits arterial blood pressure ranged from 73 to 103 $\mathrm{mmHg}$ and heart rate from 306 to 333 beats/minute, respectively (Kozma, 1974).

Before surgery, blood was collected from a central ear artery to determine hematological variables and in some experiments rheological variables. Some relevant hematological variables measured in the rabbits during the various experiments are shown in Table 3.2. For comparison, a compilation of data from the literature is given as well.

\section{Mesentery preparation}

The mesentery has a thin and transparent structure, which favours intravital microscopic observation. A picture of this preparation is shown in Figure 3.1. Arterioles, venules and arteriolo-venular shunts, can be visualized excellently. A feature of the rabbit mesentery is that rhythmic diameter variations (vasomotion) are not commonly seen: we never observed vasomotion on any of the preparations. Since flow and diameter are rather stable over the periods of time of our experiment, this preparation lends itself very well for our studies on platelet rheology in vivo.

A short segment of the distal ileum was brought outside through a small abdominal midline incision. The mesentery was spread over a siliconized glass plate mounted on the stage of a Leitz intravital microscope. During the experiments the mesentery was superfused continuously with a buffered Tyrode solution. The composition of this solution is given in table 3.3 and compared with that of blood plasma in rabbits and human. Note the 
Table 3.2: Values of some hematological variables in New Zealland white rabbits. Means are given, and, if available, standard deviations or ranges (within parentheses).

\begin{tabular}{|c|c|c|c|c|c|c|}
\hline & & $\begin{array}{c}\mathrm{Hb} \\
(\mathrm{mmol} / \mathrm{l})\end{array}$ & $\begin{array}{l}\mathrm{Ht} \\
(\%)\end{array}$ & $\begin{array}{c}\text { WBC } \\
\left(10^{3} / \mu 1\right)\end{array}$ & $\begin{array}{l}\text { Platelets } \\
\qquad\left(10^{9} / 1\right)\end{array}$ & $\mathrm{N}^{*}$ \\
\hline Kozma, & 1974 & $7.2 \pm 0.7$ & $36 \pm 37$ & $7.3 \pm 2$ & 335 & $160,182,179,00$ \\
\hline Tangelder, & 1985 & -2 & $33(28-39)$ & -- & 405 & $000,010,000,10$ \\
\hline Tangelder, & 1986 & -- & 38 (32-39) & -- & 352 & $000,007,000,07$ \\
\hline O. Egbrink, & 1988 & -. & $38(34-4 I)$ & -- & 497 & $000,013,000,13$ \\
\hline Tangelder, & 1991 & -- & - & 5.9 & -- & $000,000,050,00$ \\
\hline Schalm, & 1975 & $8.0 \pm 0$ & - & $8.7 \pm 3$ & $372 \pm 131$ & $020,000,020,20$ \\
\hline Woldhuis, & thesis & $8.8 \pm 0.8$ & $39 \pm 2$ & $6.1 \pm 3$ & $395 \pm 117$ & $046,063,016,58$ \\
\hline
\end{tabular}

- Numbers of animals for the 4 variables, respectively.

relatively high osmolarity of the Tyrode solution used. The high osmolarity helps to prevent tissue oedema, without the necessity of adding albumin to the superfusion solution. The bowels were kept moist with overlying wet gauze.

\section{Microscope}

A Leitz intravital microscope was used for all observations and video recordings. The microscope was adapted to telescopic imaging (Slaaf et al, 1982), which allows great flexibility in the location of the plane of observation. Salt water immersion lenses were immersed directly into the superfusion solution. This virtually eliminates variations in focal position due to differences in thickness of the layer of superfusion fluid, because differences in refractive index can be neglected, since the composition of the superfusion fluid differs only slightly from that of blood plasma (see table 3.3). These lenses have a great brightness, i.e. a high numerical aperture for the given magnification and, hence, a high light-gathering power. This is advantageous because of the low light levels involved in fluorescence microscopy (see below). For transillumination, a tungsten lamp was used. Microscopic images were recorded through a high sensitivity TV-camera, displayed on a monitor and stored on videotape for off-line analysis. Measurement of vessel diameters was performed off-line, using a home-built video image shearing device (Intaglietta, 1973). 


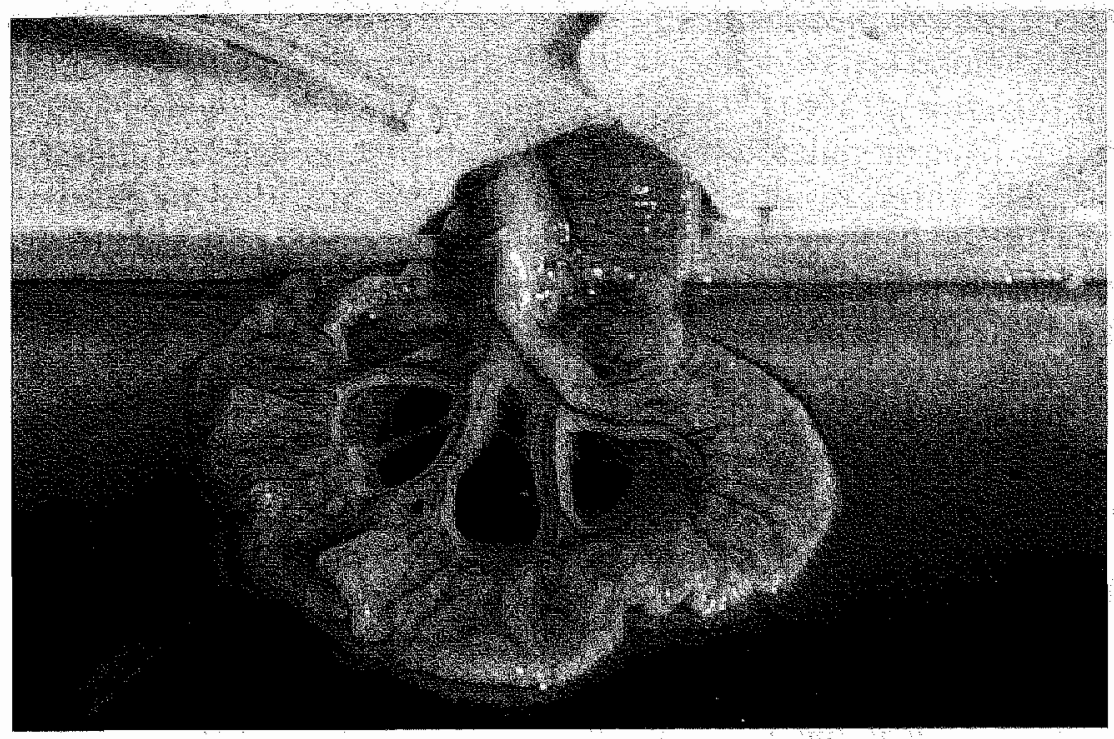

Figure 3.1: Exposed rabbit mesentery

Table 3.3: Osmolarity (in mosmol/l) and electrolyte composition (in $\mathrm{mEq} / \mathrm{l}$ ) of Lactetrol, buffered Tyrode solution and blood plasma of rabbits and adult humans.

Lactetrol Tyrode solution Rabbit plasma Human plasma*

\begin{tabular}{lrccc}
\hline $\mathrm{Na}^{+}$ & 142 & 156.4 & 131.5 & 143 \\
$\mathrm{~K}^{+}$ & 5 & 5.6 & 4.0 & 5.0 \\
$\mathrm{Ca}^{2+}$ & 5 & 5.8 & 7.0 & 5.0 \\
$\mathrm{Mg}^{2+}$ & 2 & 1.2 & 1 & 2.0 \\
$\mathrm{Cl}^{-}$ & 109 & 143.8 & 108.7 & 103 \\
lact & 45 & - & $?$ & 6 \\
$\mathrm{HCO}_{3}^{-}$ & -- & 25 & $?$ & 27 \\
$\mathrm{H}_{2} \mathrm{PO}_{4}^{-}$ & - & 1.4 & $?$ & 2 \\
glucose $_{\text {succrose }}^{-}$ & -- & 11 & $?$ & 5 \\
proteins & -- & 13 & $?$ & - \\
\hline Osmolarity & 308 & -- & $?$ & 315 \\
\hline
\end{tabular}

ref: Physiologie des Menschen. R.F. Schmidt und G. Thews, 1980 unknown values 


\section{Blood cell velocity measurements}

Red blood cell velocities were measured on-line, using a prism grating system (Slaaf et al, 1981). The three-stage prism grating serves as a spatial filter. The light modulated by the moving red blood cells is filtered by the grating. The three resulting continuous signals are used to calculate the mean velocity. The method is relatively independent of a lateral tissue movements because the width of the sensing area can be adjusted in such a way that the vessel remains within the sampling area during such movements.

\section{Fluorescence microscopy}

Fluorescence microscopy is often used to enhance the contrast of specific structures of interest, for example a cell labelled with a fluorescent dye. The staining of leukocytes, platelets or a limited number of red blood cells with such a dye allows detection of individual cells flowing in a microvessel amidst the mass of unlabelled red blood cells (Tangelder et al,1987).

Illuminated fluorescent molecules absorb radiation of a wavelength contained within a certain band spectrum, the so-called absorption spectrum. The energy of such a molecule will be raised by promoting an electron to a higher energy state, the so-called excitation state. This excitation state persists only for a short period of time, usually in the order of nanoseconds. Subsequently, the electron returns to a lower energy state while emitting light. Since, the energy freed as light by the return to the lower energy state is usually lower than the excitation energy, the emitted radiation is of a longer wavelength than the excitation radiation.

In fluorescence microscopy incident illumination shown schematically in Figure 3.2 is often used. Light passes an excitation filter to select a band that has considerable overlap with the absorption spectrum of the fluorescent dye. This is reflected in part by a dichroic mirror into the objective lens. The dichroic mirror acts as a cut-off filter. All light of longer wavelength is transmitted through the dichroic mirror and caught by a lightstop. When viewed with a microscope the light coming from the tissue consists of backscattered light of the same wavelength as that of the illumination bundle and fluorescent light, which is of a longer wavelength, but may have some overlap. The light coming back from the preparation is gathered by the objective lens and again split by the dichroic mirror: most of the backscattered excitation light is reflected towards the light source, while the remainder is caught in the barrier filter. The emitted light of 


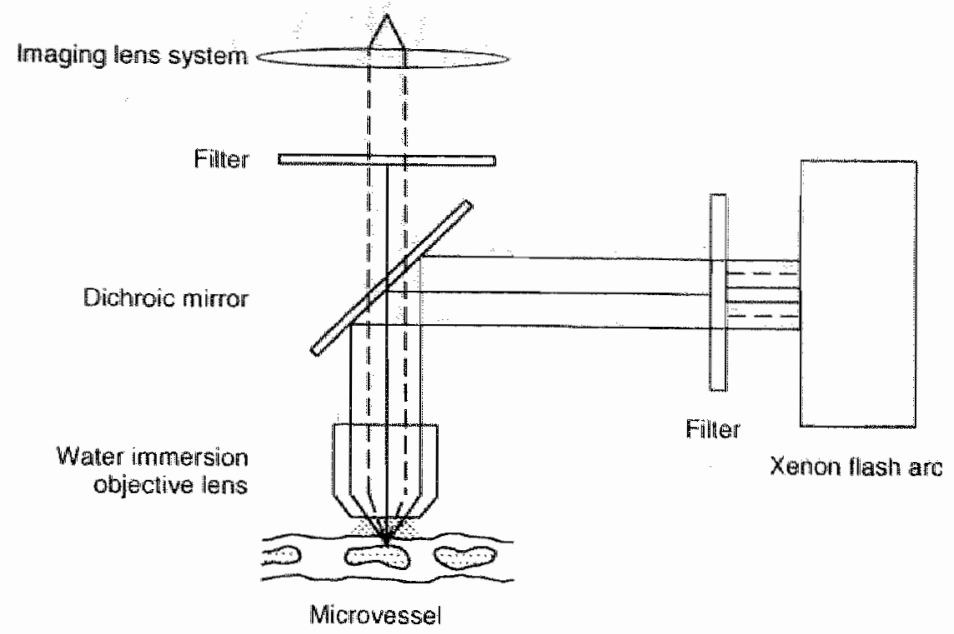

Figure 3.2: Schematic representation of the fluorescence microscope using epi-illumination.

longer wavelength (fluorescent light) is largely transmitted through the dichroic mirror and barrier filter.

When exposed to excitation light, fading of fluorescence may occur as a result of photodecomposition of the fluorescent substance. To minimize fading of fluorescence, flashes of short duration $(<0.1 \mathrm{msec})$ were used. A Xenon lamp fed by a Strobex power supply was used for dyes with longwavelength (blue or green) excitation. Xenon lamps are the most efficient light sources in this case.

\section{Visualization of platelets labelled with acridine red}

In the microcirculation individual, flowing platelets can be observed in capillaries and when located near the vessel wall in venules and arterioles using regular bright field microscopy (Schmid-Schönbein and Zweifach, 1975). However, in arterioles and venules detection of individual platelets flowing in the vessel center is hampered by the presence of many red blood cells.

After in vivo labelling with the fluorescent dye acridine red (Tangelder et al, 1982a), visualization and recording of individual, fast flowing platelets in microvessels is possible using fluorescence microscopy. White blood cells and the vessel wall are labelled by this dye as well. A sharp and nonsmeared image of the platelet is obtained by using illumination with flashes 
of short duration. In general platelets move so fast that they will appear in only one TV-frame. By giving pairs of flashes with a short time interval (1$10 \mathrm{~ms}$ ) between the two flashes, two images of the same platelet can be obtained in one TV-frame (Tangelder et al, 1986). From displacement of the two images of the same platelet and time interval between the two flashes velocity of the platelet can be assessed.

Acridine red is not an acridine derivative, but a pyronine (Lillie, 1977). The structure of acridine red is shown in Figure 3.3. Pyronines are basic dyes characterized by the presence of two alkylated or partly alkylated amino groups para to the central methane carbon on the two benzene rings.

Working solutions of acridine red were made by dissolving $75 \mathrm{mg}$ of the dye in $1 \mathrm{ml}$ physiological saline containing ethanol $(50 \% \mathrm{v} / \mathrm{v})$. After shaking, the mixture was further diluted with physiological saline to a final concentration of $7.5 \mathrm{mg} / \mathrm{ml}$. This solution was filtered through a $0.22 \mu \mathrm{m}$ filter (Cathivex). A fresh solution was made on the day of the experiment.

For each experiment two $\mathrm{ml}$ of the dye were injected into the marginal ear vein of the rabbit within 50-60 seconds. One to 3 injections of acridine red were given per animal, with an interval of 15-20 minutes between two injections. After injection of the dye, flowing platelets could be observed with sufficient intensity during 2-8 minutes by intravital fluorescence microscopy. In Figure 3.4 the flashed picture of a microvessel with some labelled platelets is shown. Acridine red used in the concentration mentioned above, did not influence either hematocrit or systemic platelet count. With this procedure nearly all circulating platelets are labelled without activation of the platelets by the dye or induction of gross ultrastructural changes in the platelets. In addition, their capability to adhere and aggregate is maintained (Tangelder et al, 1982a).

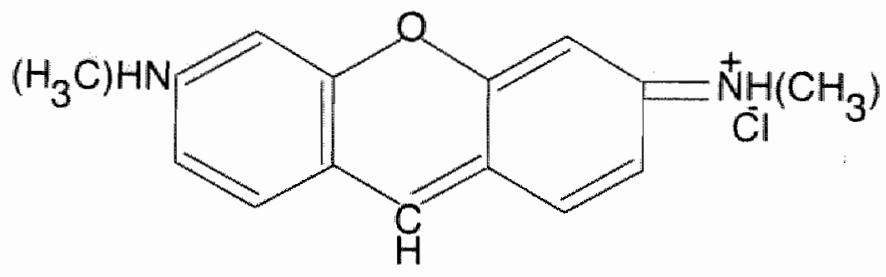

Figure 3.3: Structure of acridine red (dimethyl[6-(metylamino)-3H-xanthen-3-yliden] ammonium chloride), molwt 274.7 


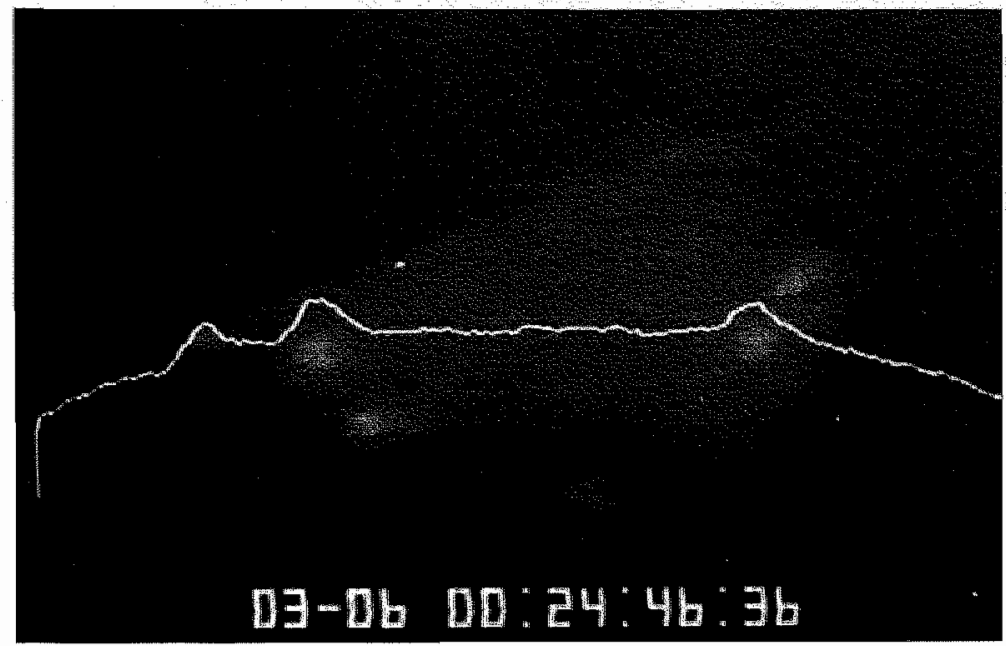

Figure 3.4: Acridine red labelled platelets flowing in a venule. The intensity along the video line through three platelets is shown. Picture taken from video monitor.

\section{Localization of platelets within an optical section}

For studies on the rheological behavior of platelets in the microcirculation, it is essential to know their radial position in the vessel. The position perpendicular to the optical axis can be easily assessed and is hardly affected by the sharpness of the image. Assessment of the position along the optical axis, however, is critically dependent on depth of field of the optics and sharpness of the image of the particle (James, 1976). Experiments performed by Tangelder and colleagues (1982b) demonstrated that platelets could be localized along the optical axis within a thin optical section. This is shown schematically in Figure 3.5. In our study we positioned sharp focus in the median plane of a vessel, i.e. the focal position yielding the widest vessel diameter. The optical section $(5-7 \mu \mathrm{m})$ was about this focal plane.

To localize a platelet within this optical section, the TV fields of the video recordings were digitized ( 512 by 512 pixels) by image processing on a personal computer (IBM). The intensity of each pixel could range from 0 (black) to 255 (white). To localize a platelet within the optical section, the intensity curve of the video line running through the center of the image of a platelet and the lumen of the vessel was plotted (see Figure 3.4). A platelet was considered to be within the optical section when the intensity peak of the platelet exceeded the envelope of the background noise. 


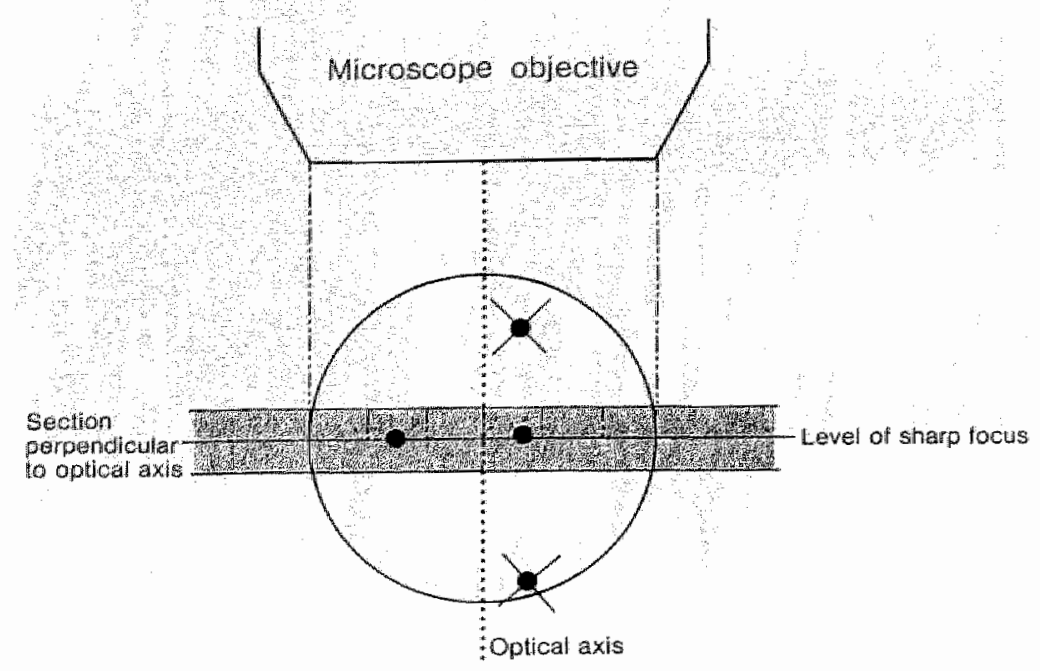

Figure 3.5: Schematic representation of a thin optical section about the median plane of a microvessel using fluorescence microscopy. Only platelets located within this optical section were used for analysis.

\section{Assessment of platelet distributions and velocity profiles}

Video recordings made to assess these two parameters were analyzed off-line. According to the direction of flow, the left and right vessel walls were drawn on a transparency positioned over the monitor screen. A vessel segment was selected with a length of approximately $27 \mu \mathrm{m}$. The centroids (the centers of gravity) of the images of platelets present within the optical section and within the marked segment during the period of analysis were marked. For each platelet marked, the distance (d) between its centroid and the left vessel wall, as well as the vessel diameter at that specific point (2R), were determined and the relative position of the platelet $(d / 2 R)$ was calculated. The relative platelet positions yield the relative distribution of platelets across the vessel width.

In case double flash is used, two images of the same platelet are obtained in one TV-frame. These two images will be displaced over a certain distance for the given time interval between the flashes allowing measurement of the velocity of the platelet and assessment of the velocity profile. The relative radial position was assessed as the mean of the two positions used to calculate the velocity. 


\section{Dextrans}

As mentioned in chapter 2, dextrans are long chains of 1-6 and 1-4 linked glucose units, that alter the aggregation tendency of red blood cells. In this thesis several dextrans were used. A $500 \mathrm{kD}$ dextran charged negatively with sulfate groups (17\% of total weight; Dx500S) was obtained from Pharmacia Fine Chemicals (Uppsalla, Sweden). From Sigma Chemical Co (St. Louis, Mo., USA) we purchased two neutral dextrans with an average molecular weight of $485 \mathrm{kD}(\mathrm{Dx} 500)$ and $39 \mathrm{kD}$ (Dx40). All dextrans were injected intravenously at a dose of $30 \mathrm{mg} / \mathrm{kg}$ bodyweight.

Dextran concentrations were determined in heparinized plasma by cleaving the dextrans into glucose with acid hydrolysis in $1 \mathrm{~N} \mathrm{HCl}$ during 3 hours at $100^{\circ} \mathrm{C}$. Subsequently, the glucose concentration was assessed with the o-toluidine method (Hultman, 1974). It is based on condensation of glucose with this aromatic amine to form a colloured product, which can be measured spectrophotometrically with monochromatic light at $630 \mathrm{~nm}$. Basic glucose levels were determined without acid hydrolysis and were subtracted from the values obtained with acid hydrolysis. For sulfated dextrans this method cannot be used, therefore we could not determined their concentration.

\section{Red blood cell aggregation}

This variable was determined in a cone-plate aggregometer (Myrenne, $\mathrm{GMBH}$, Aachen, Germany). In the M1 mode, the system rotates the blood at a shear rate of $600 \mathrm{~s}^{-1}$ for 10 seconds, followed by a shear rate of $5 \mathrm{~s}^{-1}$ for 3 seconds. The transition between the two shear levels is used to measure the rate aggregate formation. The transmission of infrared light is measured with photosensors during aggregate formation and expressed in arbitrary units. The extent of aggregation is expressed in arbitrary units. Because of the considerable scatter, 5 measurements were performed on each sample and the mean value was taken of the 3 central values.

\section{Red blood cell deformability}

This variable was measured with a Laser Optical Rotational Deformability meter (Hardeman and Rie, 1991). In this system a laser beam is passing an red blood cell suspension, which has been brought into a gap between an inner stationary cylinder and an outer rotating cylinder. The diffraction pattern is monitored by a videocamera linked to a personal computer. The above mentioned pattern is circular for cells at rest and 
becomes elliptical when the cells are subjected to shear. Direct measurements of the diffraction pattern provide both its long axis $(A)$ and its short axis (B). Red blood cell deformation is then calculated from these axis as an elongation index (EI), where $\mathrm{EI}=\mathrm{A}-\mathrm{B} / \mathrm{A}+\mathrm{B}$ (Hardeman et al, 1988).

\section{Plasma viscosity}

This variable was measured with a capillary viscometer (Rheomed GMBH, Aachen, Germany). At a constant driving pressure of $0.1 \mathrm{kPa}$, a given plasma sample is forced to flow through a polyethylene tube with a constant diameter of $800 \mu \mathrm{m}$. According to Poiseuille's law (see chapter 2), the time needed to flow through a certain length of this capillary is proportional to the viscosity of the newtonian fluid. The system was calibrated against the time measured for a fluid of known viscosity. Temperature control is essential and was set at $37^{\circ} \mathrm{C}$ throughout the measurements. Two measurements were performed on each plasma sample and the average value was taken. 


\section{CONCENTRATION PROFILE OF BLOOD PLATELETS DIFFERS IN ARTERIOLES AND VENULES}

\subsection{INTRODUCTION}

Blood platelets play a key role in the reaction of blood to vessel wallinjury, i.e. in hemostasis and thrombosis (Tangelder et al, 1989). Experiments performed in vivo in rabbit mesenteric arterioles showed the platelet concentration near the wall to be about twice the concentration in the center of the vessel (Tangelder et al, 1985). Margination of platelets is also surmised in renal interlobular arteries of the cat (Ofjord and Clausen, 1986). These results are comparable to findings obtained in vitro,using blood suspensions flowing through glass channels (Eckstein et al,1988; Tilles and Eckstein, 1987) or slits (Palmer, 1967). The presence ofred blood cells seems to play an important role in the observed non-uniform distributionof blood platelets (Palmer, 1967; Tilles and Eckstein, 1987; Turitto and Baumgartner, 1975).

Venules differ from arterioles in their sensitivity to monoamines (Heltianu et al, 1982), for example with regard to permeability (De Clerck et al, 1985), and in their thrombo-embolic reaction to microinjury (oude Egbrink et al, 1988). These findings suggest a difference in endothelial cell function between these microvessels, possibly influencing platelet-vessel wall. interactions. Another difference between both vessel types is the presence of leukocyte margination. Rolling and sticking leukocytes are often seen invenules but seldom in arterioles (Atherton and Born, 1972; House and Lipowsky, 1987; Tangelder and Arfors, 1991). This might also affect platelet vessel wall interactions and, hence, the concentration of these blood cells near the wall. It is therefore uncertain whether the platelet distribution found in arterioles can be extrapolated to venules. In the present study the platelet distribution in venules was studied in vivo and compared with that

* The study described in this chapter has been published in Am J Physiol 262:H1217-1223, 1992 
in arterioles. Because a striking difference in platelet distribution between these microvessels was observed, experiments were performed to evaluate the influence of leukocyte margination, if any, on platelet distribution in venules.

\subsection{MATERIALS AND METHODS}

\section{Animals and intravital microscopy}

Fifteen New Zealand white rabbits of either sex and ranging in weight from 2.0 to $3.0 \mathrm{~kg}$ (mean $2.6 \mathrm{~kg}$ ) were used. Anesthesia was induced by intramuscular injections of ketamine hydrochloride $(40 \mathrm{mg} / \mathrm{kg}$ body weight, Nimatex, A.U.V., Cuijk, the Netherlands) and xylazine hydrochloride (4 $\mathrm{mg} / \mathrm{kg}$ body weight, Sedamun, A.U.V.). Anesthesia was maintained with a continuous intravenous infusion $(15 \mathrm{ml} /$ hour $)$ of the same substances, 40 and $5 \mathrm{mg} / \mathrm{kg}$ bodyweight/hour respectively, dissolved in a Lactetrol solution (Aesculaap, Boxtel, the Netherlands). This infusion was given through a polyethylene catheter (PE 60) in the femoral vein, starting about 60 minutes after induction.

After induction of anesthesia, blood was collected from a central ear artery in disodium ethylenediamine tetraacetic acid (EDTA, $0.1 \mathrm{vol}$, $0.027 \mathrm{M}$ ). Platelets were counted twice with a Coulter Counter (model ZF) and hematocrit measurements were performed in duplicate with an Autocrit II centrifuge (Clay Adams). A polyethylene catheter (PE-60), positioned in the femoral artery and connected to an external pressure transducer (CTC, CP-01), was used to measure mean arterial blood pressure. Heart rate was derived from the instantaneous pressure pulses. Both signals were recorded continuously on a physiological recorder (Schwarzer, US 266). Throughout the experiments mean blood pressure was $78 \pm 3 \mathrm{mmHg}$ (mean $\pm \mathrm{s}$.d.) and heart rate $140 \pm 17$ beats per minute. The blood pressure catheter was continuously perfused with saline $(5 \mathrm{ml} /$ hour) via an Intraflo II-system (Sorenson Research); no heparin was used. The trachea was cannulated and the cannula ( $4.5 \mathrm{~mm} \mathrm{ID}$, Mallinckrodt) connected to an infant ventilator (Loosco, $\mathrm{mk} 2$ ). The rabbits were ventilated with a mixture of air and oxygen $\left(3 \mathrm{l} /\right.$ minute, total volume; 0.2 to $0.3 \mathrm{l} / \mathrm{minute} \mathrm{O}_{2}$ ). The inspiration:expiration ratio was set at $1: 1.5$ and the tidal volume was adjusted to approximately $20 \mathrm{ml}$, depending on the blood gas values. Blood gas analyses were performed every 30 minutes in blood collected from a central ear artery, using an acid-base analyzer ( $A B L 3$ Radiometer). Mean values ( \pm s.d.) were pH $7.45 \pm 0.1, \mathrm{pCO}_{2} 34.6 \pm 4.8$ and $\mathrm{pO}_{2} 89.3 \pm 7.8 \mathrm{mmHg}$. The 
latter two parameters did not show a consistent trend during the experimental period of about 1.5 to 2 hours, while $\mathrm{pH}$ decreased by 1.2 to $1.9 \%$

A short segment of the distal ileum was brought outside through a small abdominal midline incision. The mesentery was spread over a siliconized glass plate mounted on the stage of a Leitz intravital microscope adapted to telescopic imaging (Slaaf et al, 1982). During the experiments the mesentery was superfused continuously with buffered Tyrode solution (pH $7.37 \pm 0.04 ; 37^{\circ} \mathrm{C}$ ). The bowels were kept moist with overlying wet gauze.

To visualize the circulating blood platelets (see below), a Leitz SW 50x water-immersion objective (numerical aperture 1.0 ) and $2 \mathrm{X}$ projection eyepiece were employed. Fluorescence microscopy was performed with an incident illuminator (Leitz Ploemopak 2.2 with interchangeable filter set $\mathrm{N}$ 2.1, tubefactor 1.6x, excitation filter: BP 515-560, dichroic mirror: RKP 580, barrier filter: LP 580). As light source a Xenon flash arc fed by a Strobex power supply (Chadwick Helmuth) was used. The images were recorded through a high sensitivity TV-camera (Bosch TYC 9 A; one inch SIT tube, RCA $4804 \mathrm{HP}$ ) and stored on videotape. The final magnification at the front plane of the TV camera was $200 \mathrm{X}$. To count the number of leukocytes rolling in a venule, transillumination and an $1 \mathrm{X}$ projection eyepiece were used (final magnification $100 \mathrm{X}$ ). Local vessel diameters were measured from video images using a home-built image shearing device according to Intaglietta and Tompkins (1973).

To investigate a difference in shape between arterioles and venules, if any, a mesentery was fixed in situ by superfusion with $2.5 \%$ glutaraldehyde and $4 \%$ paraformaldehyde in $0.1 \mathrm{M}$ phoshate buffer $(\mathrm{pH} 7.3)$ during 10 minutes. After excision, fixation was continued in these solutions during one week at $4^{\circ} \mathrm{C}$. Postfixation in $1 \% \mathrm{OsO}_{4}\left(60\right.$ minutes, $\left.4^{\circ} \mathrm{C}\right)$, dehydration through graded alcohol and embedding in Epon were performed as described in detail elsewhere (Peeze-Binkhorst et al, 1989). In hematoxylin and eosin stained thin sections $(1 \mu \mathrm{m})$, the eccentricity of the microvessels, i.e. the ratio short axis/long axis, was measured. Vessels containing one or more leukocytes touching the wall were considered as venules.

\section{Platelet concentration distribution assessment}

Segments of venules and arterioles ranging in diameter from 15 to 33 $\mu \mathrm{m}$ were selected in such a way that no branch points and no adhering leukocytes could be observed over a length of at least 10 vessel diameters. Experiments were performed with position of sharp focus in the median 
plane of the vessel, i.e. the focal position yielding the widest vessel diameter.

To visualize the individual flowing platelets, $2 \mathrm{ml}$ of a solution of the fluorescent dye acridine red $(7.5 \mathrm{mg} / \mathrm{ml})$ was injected through a polyethylene catheter in a marginal ear vein (Tangelder et al, 1982a). With this procedure nearly all platelets are labelled without activation of the platelets by the dye or induction of gross ultrastructural changes. In addition, their capability to adhere and aggregate is maintained (Tangelder et al, 1982a). Leukocytes and the vessel wall are labelled as well, but red blood cells are not. One to 3 injections of acridine red were given per animal, with an interval of 15-20 minutes between two injections. After each injection the circulating platelets were recorded over 5-7 minutes by fluorescence video microscopy.

Flashes of short duration $(<0.1 \mathrm{~ms})$ were given every 6 th TV field in the blanking period of the TV-camera, which corresponds to a flash interval of $120 \mathrm{~ms}$. By using a flash of short duration, a smeared image due to platelet movement was prevented. With this interval between flashes, we never detected a platelet that had just entered the field of view $(39 \times 55 \mu \mathrm{m})$ in the next image. The majority of the vessels analyzed were oriented with their axis along the diagonal of the TV-screen. Hence it can be calculated that even platelets flowing near the vessel wall were likely to move at a velocity of at least $0.5 \mathrm{~mm} / \mathrm{second}$.

Off-line analysis was done on still video fields. To obtain a platelet distribution in a vessel, $150-160$ TV fields were analyzed, covering a time period of approximately 20 seconds. Because each flashed TV field represents an instantaneous picture, showing the distribution at that moment, a possible influence of differences in platelet velocity can be neglected.

Only platelets flowing within a thin optical section $(5$ to $7 \mu \mathrm{m})$ were used for analysis (Tangelder et al,1982b). To localize a platelet within this optical section, the TV fields of the video recordings were digitized (512 by 512 pixels) by image processing on a personal computer (IBM). The intensity of each pixell could range from 0 (black) to 255 (white). In a band of 3 pixels wide, drawn through the center of the image of a platelet and the lumen of the vessel, the intensity curve was plotted. A platelet was considered to be within the optical section when the intensity peak of the platelet exceeded the background noise. The thickness of the optical section remains constant over the whole vessel width (Tangelder et all, 1985).

As is illustrated in Figure 4.1A, the centroid of the images of the platelets present within the optical section during the period of analysis were marked on a transparency positioned over the monitor screen. 
According to the direction of flow, the left and right vessel walls were drawn and a vessel segment selected with a length of approximately $27 \mu \mathrm{m}$. For each platelet marked, the distance (d) between the centroid of the platelet and the left vessel wall, as well as the vessel diameter at that specific point $(2 R)$, were determined and the relative position of the platelet $(d / 2 R)$ was calculated. In the different vessels, the number of platelets counted, ranged from 63 to 144 (mean $112 \pm 26$ ) in arterioles and from 60 to 259 (mean 148 $\pm 62)$ in venules.

\section{Inhibition of leukocyte mangination}

In venules leukocytes often move along the endothelium at a velocity significantly lower than that of other blood cells. The influence of these rolling leukocytes on platelet distribution was investigated by inhibition of leukocyte rolling with dextran sulfate (Tangelder and Arfors, 1991). Six rabbits received an intravenous injection of a dextran sulfate $(30 \mathrm{mg} / \mathrm{kg}$ bodyweight) with molecular weight of 500.000 and a sulfate content of $17 \%$ (Pharmacia, Uppsala, Sweden). This dose has been reported to inhibit leukocyte rolling almost completely, i.e. $>90 \%$ for at least 2 hours (Tangelder and Arfors, 1991).

\section{Statistical analysis}

For presentation of the platelet concentration profiles relative frequency distributions were used. An example of this distribution for the experiment shown in Figure 4.1A is presented in Figure 4.1B. To obtain such a histogram, the platelets were assigned to 20 segments, according to their relative position along the width of the vessel. The number of twenty segments was chosen to obtain an estimate of the general distribution (see below) with sufficient detail given the number of platelets counted. To obtain a general frequency distribution for both groups of vessels, individual histograms were pooled. Before pooling, each individual distribution of counts (left $\mathrm{Y}$-axis in Figure 4.1B) was converted into a density distribution, which was subsequently normalized with respect to the mean density in that vessel (right $\mathrm{Y}$-axis in Figure 4.1B; see appendix). This is necessary for proper comparison between arterioles and venules, since their distributions differed and different segments contributed to the mean density to a different extent. The $95 \%$ confidence limits of the general distributions were calculated using the standard error of the mean (Daniel, 1983).

All parameters are presented as ranges and/or means with standard 
deviations. To test for a difference between two groups, the Mann-Whitney $U$ test was used (Siegel, 1956). Correlations were performed with the nonparametric Spearman rank correlation test (SPSS-PC statistical package, SPSS Inc, Chicago, USA). When the p-value was equal to or exceeding 0.05 , a difference was considered as statistically non-significant.

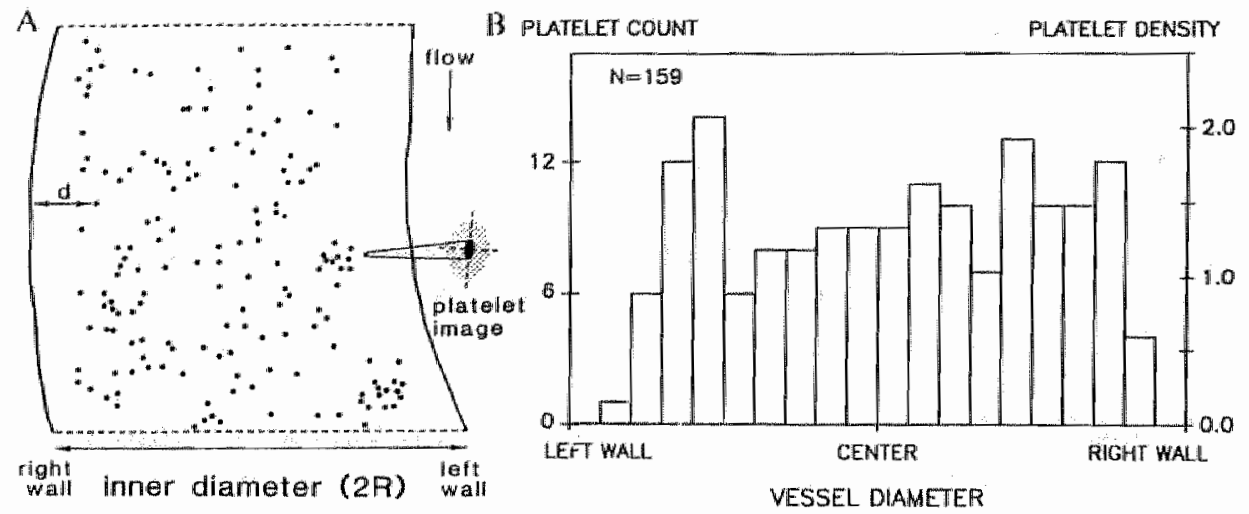

Figure 4.1: (A) Schematic presentation of platelet centroid positions (dots) present within the optical section in $160 \mathrm{TV}$-fields in a control venule, i.e. no dextran sulfate used; $(B)$ concomitant platelet count histogram (left $Y$-axis) and normalized distribution (right $Y$-axis). An image of a platelet (shaded) and representation of its centroid is shown schematically in $A ; d=$ distance between the centroid of a platelet and the left wall. Vessel diameter = $30.9 \mu \mathrm{m}$.

\subsection{RESULTS}

The hematocrits of the 15 animals used ranged from 34.4 to $41.7 \%$ $(38.6 \% \pm 2.1)$ and the electronic platelet counts from 241 to $534 \times 10^{\%} / 1$ $\left(407 \times 10^{\circ} / 1 \pm 94 ; 14\right.$ animals). These values are within the normal ranges for rabbits (Kozma, 1974).

The diameter of the 21 venules used ranged from 17 to $31 \mu \mathrm{m}$, with no significant difference between venules in which leukocyte rolling had been inhibited by dextran sulfate $(23.4 \mu \mathrm{m} \pm 3.7 ; \mathrm{n}=10)$ and the control venules $(22.6 \mu \mathrm{m} \pm 4.1 ; n=11)$. In the 10 arterioles analyzed the diameter ranged from 15 to $33 \mu \mathrm{m}(21.5 \mu \mathrm{m} \pm 6.5)$. In none of the preparations we observed changes in vessel diameter, for example, due to vasomotion (Tangelder et al, 1984). 
In transillumination a thin zone could be discerned near the wall of both arterioles and venules, which was clearly brighter than the rest of the lumen. In 8 arterioles and 7 control venules these zones were outlined on a transparency and their width measured. The mean width, expressed as percentage of the vessel radius, ranged from 8.4 to $18.4 \%(14.3 \% \pm 3.0)$ in arterioles and from 14.5 to $25.9 \%(18.5 \% \pm 4.2)$ in venules, the difference being significant $(p=0.03)$.

The eccentricity of microvessels in fixed mesentery ranged from 0.65 to $0.90(0.76 \pm 0.08)$ in 14 venules and from 0.53 to $0.96(0.84 \pm 0.12)$ in 11 arterioles. Because cutting at an angle, for example due to tortuosity of a vessel decreases the measured ratio short axis/long axis, the highest values will yield the best indication of vessel shape. The three highest values measured were $0.86,0.87$ and 0.90 in venules and $0.92,0.95$ and 0.96 in arterioles.

\section{Platelet distributions}

Examples of platelet distributions in venules are shown in the Figures 4.1B and 4.2, and those in arterioles in Figure 4.2. These examples suggest a difference between arterioles and venules. To detect influence, if any, of either vessel diameter or total number of platelets counted in a vessel on the platelet distributions, we related in both vessel types diameter and total platelet count to the relative platelet concentration in each of the 4 segments between relative radial position $\left(R_{i} / R ; R_{i}\right.$ is the distance from the vessel center) 0.6-1.0 and in the vessel center (segments between 0.0-0.2 pooled). No correlations could be found and hence vessels were pooled to bring out more clearly a possible difference between arterioles and venules.

The general platelet density distribution with $95 \%$ confidence limits for the control venules and the arterioles normalized with respect to the mean density in the vessel, is presented in Figure 4.3. The mean value in the vessel center is $0.55 \pm 0.33$ in arterioles and $1.04 \pm 0.45$ in venules, indicating that approximately two times more platelets are located in the center of venules as compared to arterioles $(p<0.005)$.

Figure 4.3 also shows that the distributions differ near the vessel wall. Unlike in arterioles, in venules there is a relative exclusion of platelets from the segments close to the vessel wall. This difference is more clear when the left and right segments near the vessel wall are pooled. The average platelet densities close to the vessel wall, as obtained from these pooled data, are shown in Figure 4. The peak densities shown have a similar height in venules and arterioles, but their relative radial positions differ. In arterioles, 
NORMALZED PLATELET DENSITY
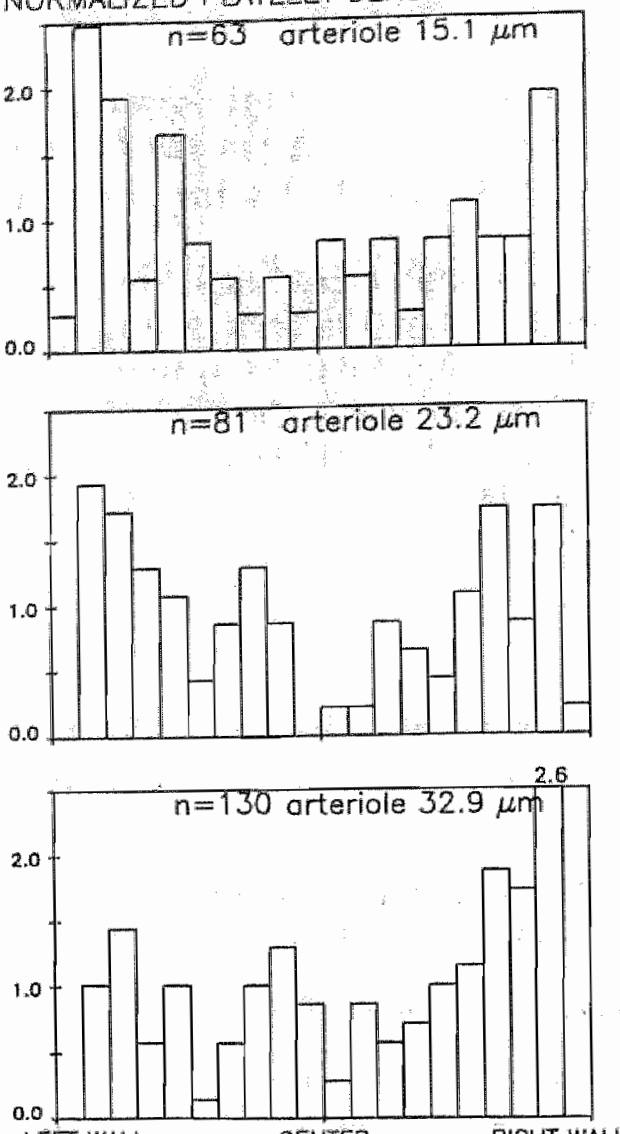

LEFT WALLL
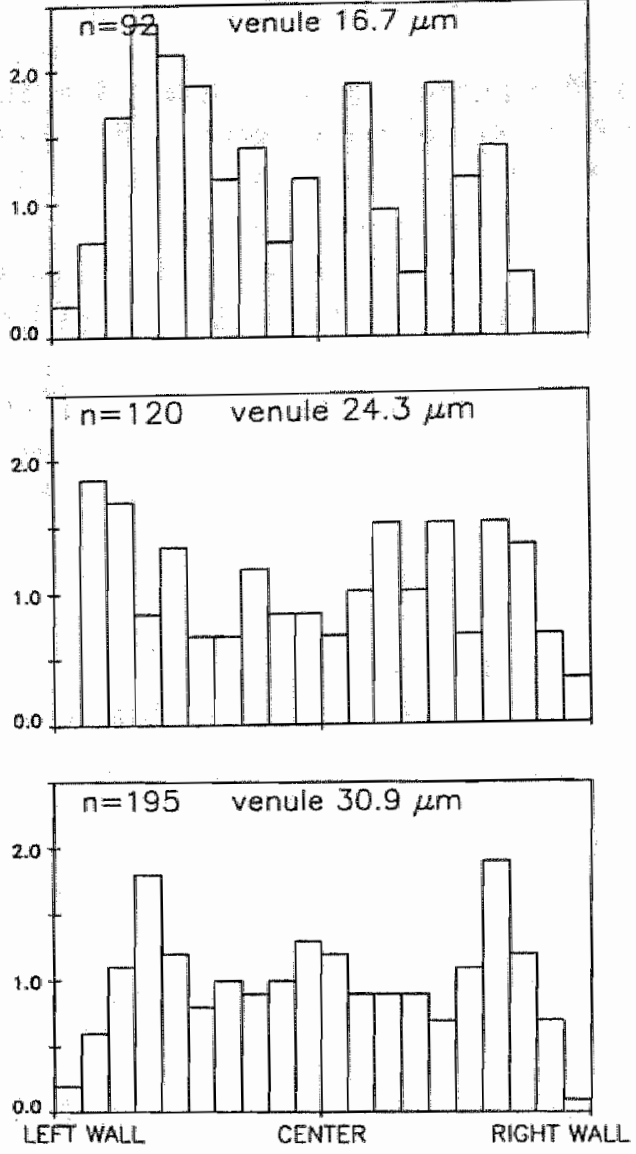

VESSEL DIAMETER

Figure 4.2: Examples of normalized platelet distributions in three arterioles (left) and three venules (right), with vessel diameters at the extremes and near the median of both vessel types. Vessel diameters and numbers of platelets counted $(n)$ are indicated.

platelet density rises steeply in the two segments close to the wall, reaching a peak relatively close to the wall (at $0.8-0.9$ of the vessel radius). In venules, this increase is less steep, reaching a peak at $0.6-0.7$ of the vessel radius. The platelet densities differed significantly between arterioles and venules in the two segments between 0.5 and $0.7(\mathrm{p}<0.02)$ and in the segment between 0.8 and $0.9(p<0.002)$ of the vessel radius. 


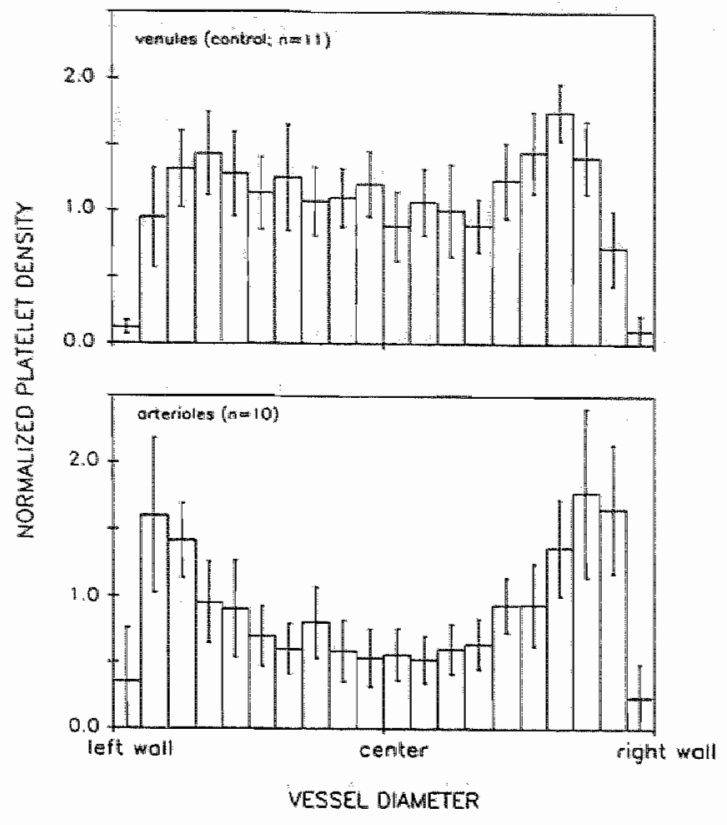

Figure 4.3: Normalized general platelet density distribution with $95 \%$ confidence limits in control venules and arterioles.

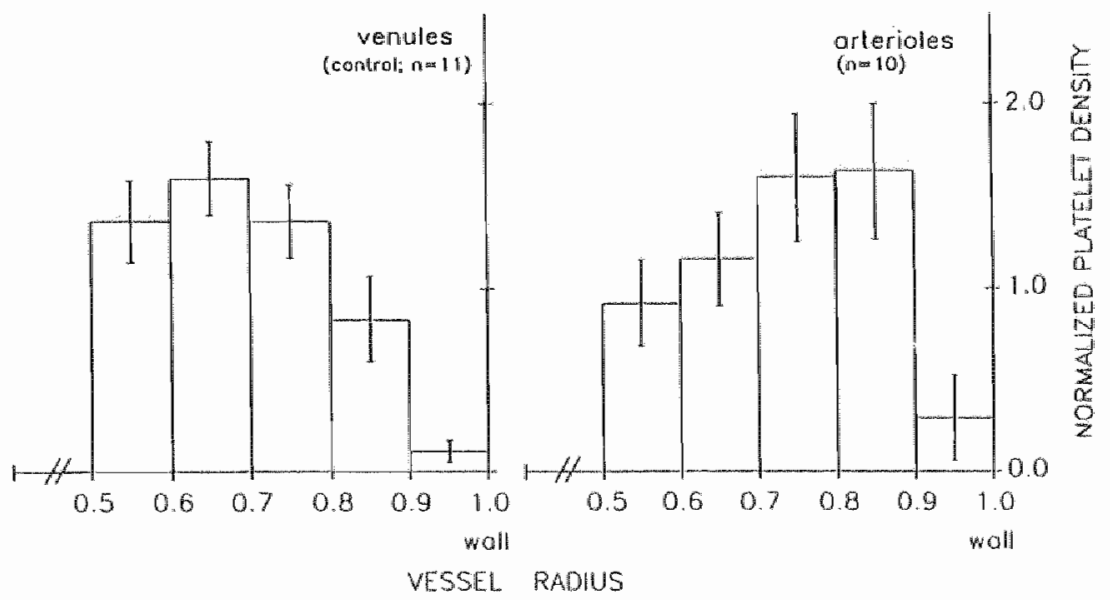

Figure 4.4: Normalized platelet densities with $95 \%$ confidence limits in the 5 segments closest to the vessel wall. Distributions obtained by pooling left and right segments. 


\section{Comparison between venules with and without rolling leukocytes}

In the control venules, the number of rolling leukocytes ranged from 21 to 85 per minute $(49 /$ minute \pm 20 ). By use of fluorescence microscopy, the depth of field for detection of rolling leukocytes was considerably smaller than vessel diameter. During assessment of the platelet concentration distribution, the average number of rolling leukocytes observed in this small depth of field and expressed per minute, ranged from 0 to 15 $(8 /$ minute \pm 5$)$. No correlations were found between leukocyte rolling and the relative platelet concentrations in each of the 4 segments between radial position $0.6-1.0$ and in the vessel center (segments 0-0.2 pooled). Leukocyte rolling was not seen in arterioles.

Treatment with dextran sulfate decreased the number of rolling leukocytes from a mean value of $54 \pm 32$ per minute (range 24-96) to a value of 0 in all vessels except two, in which on the average one rolling leukocyte per minute was observed. The general platelet concentration distribution in the dextran sulfate treated animals is shown in Figure 4.5. Statistical comparison with the venular platelet distribution shown in Figure 4.3 , indicated no significant difference between control venules and venules in which leukocyte rolling was inhibited with dextran sulfate.

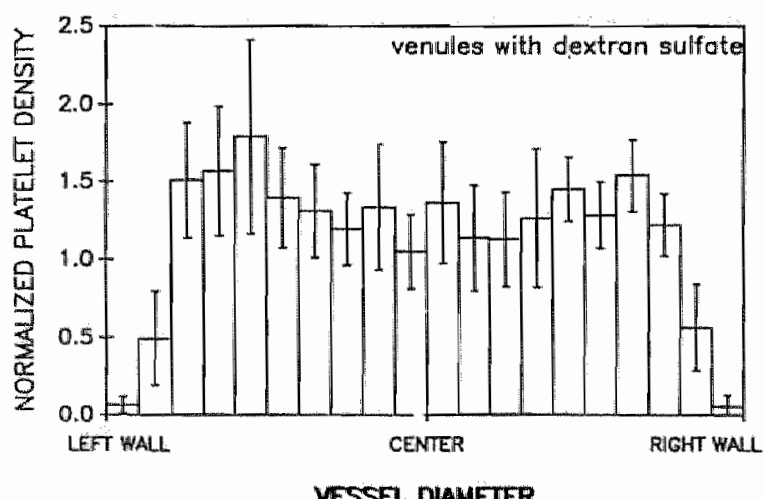

Figure 4.5: Normalized general platelet distribution in 10 venules in which leukocyte rolling was inhibited by dextran sulfate. The $95 \%$ confidence limits are shown as well.

\subsection{DISCUSSION}

The findings in the present study show that in vivo the relative platelet distribution differs greatly in venules and arterioles. First, in the center of 
the vessel the platelet density relative to the mean density is significantly higher in venules than in arterioles, while near the wall the opposite is observed. Second, close to the wall there seems to be a relative exclusion of platelets in venules, but not in arterioles. The difference in platelet distribution between venules and arterioles is not caused by the presence of marginating leukocytes in venules.

A striking difference between the venules and arterioles in the exteriorized mesentery is the presence of leukocyte margination in venules, but not in arterioles (this study; Atherton and Born, 1972; House and Lipowsky, 1987; Tangelder and Arfors, 1991). The number of rolling leukocytes observed in this study is comparable to that found in other studies performed in rabbits (oude Egbrink et al, 1992; Tangelder and Arfors, 1991). No correlation could be found between the observed level of leukocyte rolling and the platelet concentration at different radial positions. In addition, no significant difference was found between the platelet distributions in control venules and in venules in which leukocyte rolling had been inhibited by dextran sulfate. These findings indicate that the influence of rolling leukocytes on platelet distribution, for example, by forcing platelets towards the vessel center, can be neglected. It is also unlikely that the difference in platelet distribution between venules and arterioles is caused by the presence of firmly adherent leukocytes. In the present study the observations were always performed in vessel segments without sticking leukocytes within at least 10 vessel diameters.

It is unlikely that the difference in platelet distribution between arterioles and venules was caused by a difference in shape. Almost circular venules and arterioles can be found in fixed mesentery (this study; Fox et al, 1980), as well as in other tissues (Kovacs et al, 1975). Our histological findings suggest that a difference in eccentricity between arterioles and venules, if any, will probably not exceed $10 \%$. Calculation showed that a decrease in the short axis/long axis ratio from 1.0 to as much as 0.67 , barely changes the platelet distribution profile in both venules and arterioles. The differences between both distributions becomes even more accentuated. The same is found if in a circular vessel the depth of the optical section is increased by $50 \%$. This finding indicates that the differences in platelet distribution between arterioles and venules is not caused by a possible difference in section depth.

In this study the position of a platelet was determined by localizing its centroid. Therefore, the difference in platelet distribution near the wall between arterioles and venules could be explained by differences in platelet orientation in these microvessels. Although the disk shaped blood platelets 


\section{Chapter 4}

can move in apparent contact with the vessel wall (Tangelder et al, 1986), their centroid cannot come closer to the wall than about $0.5 \mu \mathrm{m}$, even if the platelets are perfectly aligned to the wall. In arterioles platelets have been shown to tend to align with flow, especially near the wall (Teirlinck et al, 1984), so that they can come relatively close to the wall in these microvessels. In venules the orientation of platelets has not been determined. If in venules platelets spend most of their time with their long axis more or less perpendicular to the vessel wall, fewer data points will be obtained in the segments closest to the vessel wall. However, this factor allone cannot account for a mean maximum density in venules at a distance of $0.6-0.7$ of the vessel radius, since the long axis of a rabbit platelet is on the average 3 $\mu \mathrm{m}$ (Teirlinck et al, 1984).

The above mentioned position of the mean maximum density in venules at $0.6-0.7$ of the vessel radius cannot be ascribed to the so called tubular pinch effect (Segre, 1962). According to this effect, rigid spheres flowing through a tube under laminar flow conditions at sufficiently high Reynolds numbers tend to move to an equilibrium position at 0.6 of the tube radius. This effect depends on inertial forces. However, in venules the Reynolds numbers are below unity. Hence viscous forces dominate and the effects due to inertia are negligible.

The platelet distributions, as found in venules in vivo, show no correspondence with those obtained in vitro. Experiments performed with blood suspensions flowing in glass channels (Eckstein et al, 1988; Tilles and Eckstein, 1987) or slits (Palmer, 1967), showed that the platelet concentration near the wall was found to be 3 to 5 times higher than that in the center of the channel. These in vitro distributions are in agreement with those found in arterioles in the present study, where the platelet concentration near the wall is approximately 3 times the concentration in the center. A higher platelet concentration near the vessel wall was also found in a previous in vivo study in arterioles (Tangelder et al, 1985).

It seems unlikely that the difference in platelet distribution between venules and arterioles is caused by a difference in wall shear rate. The findings in the in vitro study of Tilles and Eckstein (1987) indicate that the platelet distribution is independent of wall shear rate at values exceeding $430 \mathrm{~s}^{-1}$. In vivo generally higher wall shear rates are found in arterioles (Tangelder et al, 1988). It is likely that this also holds for the venules used in this study. In all venules, the platelets flowing within a distance from the wall of $1 \mu \mathrm{m}$ had moved out of the field of view at the moment of the next flash, indicating a platelet velocity of $0.5 \mathrm{~mm} / \mathrm{s}$ or higher. If a linear vellocity gradient is assumed from this radial position to zero velocity at the wall (no- 
slip condition), a least estimate of wall shear rate of approximately $500 \mathrm{~s}^{-1}$ is obtained.

The difference in platelet distribution between arterioles and venules might be related to the so-called "plasma-layer" near the wall (Kurland, 1974). However, this layer is not free of blood cells, all three types do come in apparent contact with the vessel wall (Bloch, 1962; Tangelder et al, 1986). Nevertheless, red blood cells cannot come closer to the vessel wall with their center of gravity than about $2.5 \mu \mathrm{m}$ (Tangelder et al, 1986). The wall exclusion of red cells might be somewhat smaller in arterioles than in venules. If local hematocrit near the vessel wall is higher in arterioles than in venules, one might have expected a higher platelet concentration near the wall in venules than in arterioles. However, the opposite was found. The finding that wall exclusion of both platelets and red blood cells seems more pronounced in venules than in arterioles, suggest a difference in wall structure and/or function. Differences in endothelial surface properties such as glycocalyx composition and electrical charge might be important. In capillaries, for example, it has been shown that changes in the composition of the endothelial glycocalyx influence capillary hematocrit (Desjardins and Duling, 1990). Platelets are not only essential for endothelial integrity, but they can also release substances, such as serotonin, that increase microvascular permeability and protein leakage (De Clerck et al, 1985). Because this latter influence is exerted mainly in postcapillary venules, it is tempting to speculate that in venules mechanisms exist that prevent platelets from coming too near to the wall.

The low platelet density in the center of arterioles can probably be explained by the presence of red blood cells (Palmer, 1967; Turitto and Baumgartner, 1975). It is not known whether the relatively higher density of platelets in the center of venules can be explained by a relative absence of red blood cells in this part of these vessels, because to our best knowledge hematocrit distributions over the cross-sectional area of venules have not been measured. However, the mean local hematocrit in venules does not differ from that in arterioles of comparable size (Kanzow et al, 1982; Lipowsky et al, 1980; Sarelius and Duling, 1982). The tendency of red blood cells to form aggregates might also play a role (Palmer, 1967). Red cell aggregation can occur when shear forces are low, i.e. in the center of the vessel (Tangelder et al, 1986). However, it is not known whether differences exist in red cell aggregate formation between arterioles and venules.

It is unknown what length along the vascular tree is needed for the mechanism of platelet dispersion to develop the low platelet density as 
observed in the center of arterioles. In vitro axial accumulation of red blood cells needs a length of at least $200 \mathrm{x}$ the tube diameter to reach complete development (Palmer and Betts, 1975). It is possible that the available length from systemic arteries to arterioles is large enough in this respect. However, from capillaries to small venules this length might be too short. In vessels with the size of capillaries blood cells flow in single file. Therefore, when blood from such small vessels confluence, platelet distribution might be more or less homogeneous at the beginning of the venular tree. However, if this factor plays a role, it does not explain the relative exclusion at the wall. Another factor in venules might be that blood, flowing in from side branches pushes blood cells away from the wall.

In conclusion, platelet distribution differs greatly between venules and arterioles. Platelet density in the center is significantly higher in venules than in arterioles. Near the wall platelet density is significantly higher in arterioles, while in venules a relative exclusion seems to exist close to the vessel wall. These differences cannot be explained by the presence of leukocyte margination in venules. Beside the involvement of red blood cells, the position of a vessel within the vascular network and/or differences in vessel wall properties might be important

\subsection{APPENDIX: Calculation of the (mean) platelet density in a vessel}

Platelet counts were obtained from a number of TV fields (f) of vessel parts with a length, 1 , and width, $2 R$ ( $R=$ vessel radius). By objective criteria (Tangelder et al, 1982b) we used only those platelets which were located in a thin optical section positioned about the median plane of the vessel (Figure 4.6A). The depth of the section (h) may vary between 5 and $7 \mu \mathrm{m}$ among experiments (Tangelder et al, 1982b), but is constant within an experiment over the full length and width of the vessel part under observation (Tangelder et al, 1985). The optical section was divided into 20 segments of equal width across the vessel, resulting in 20 segments of equal volume:

$$
V_{i}=h \cdot b \cdot I
$$

where $\mathrm{V}$ is volume ( $i$ denoting position of segment), $\mathrm{h}$ is section depth, $\mathrm{b}$ is width of each segment and $l$ is length of vessel part under observation. The 


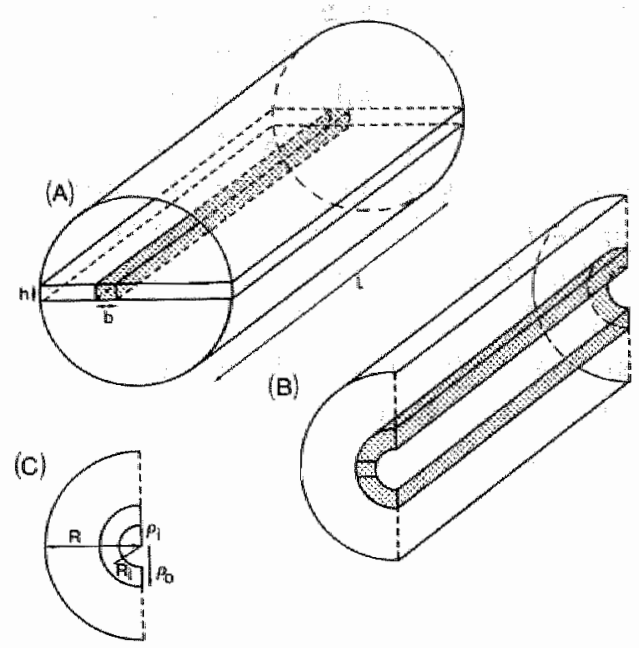

Figure 4.6: (A) Schematic representation of a vessel with optical section (section depth $=$ h). A segment within the optical section with width (b) and length (l) is shaded.

(B) Schematic of the concomitant annular semicylinder (shaded) of the segment depicted above within the optical section.

(C) $\rho_{o}=$ outer radius and $\rho_{i}=$ inner radius of semicylinder.

$R_{i}=$ distance from vessel center to center of the $i-$ th segment and $R=$ vessel radius.

number of platelets counted in each of the segments is designated $N_{i}$. Because these counts were obtained in $f$ TV fields, for each TV field the average number of platelets in each segment volume is $\mathrm{N}_{\mathrm{i}} / \mathrm{f}$. The platelet density (= number of platelets per unit volume) in each segment is defined as:

$$
D_{1}=\frac{N_{1} / f}{V_{1}}=\frac{N_{1}}{f \cdot h \cdot b \cdot 1}
$$

Because the section depth is relatively small compared with vessel diameter, the density distribution in the optical section may be considered as representative of that in the median plane of the vessel. Because the flow conditions in straight, unbranched parts of a vessel are in general cylindrically symmetric, this density distribution is representive of that along the vessel diameter. 
To compare platelet densities between various vessels, we normalized each platelet density distribution to the mean density in that vessel. The platelet counts may be asymmetrically distributed, i.e. the count in segment $i$ on the left hand side of the vessel is not necessarily equal to that of the segment at the same radial position on the right hand side $(21-i)$. Hence the density in each segment $i$ is onlly representative of that in the concomitant annular semicylinder (see Figure 4.6B). To each of the 20 segments belongs such an annular semicylinder. Note that the volume of such an annular semicylinder depends on its position. The sum of the volumes of these 20 annular semicylinders equals that of the whole part of the vessel under consideration $\left(\pi \cdot R^{2} .1\right)$. The volume of an annular semicylinder $\left(V_{R i}\right)$ with outer radius $\rho_{\mathrm{o}}$ and inner radius $\boldsymbol{\rho}_{\mathrm{i}}$ is given by:

$$
V_{R_{1}}=\frac{1}{2} \pi \cdot \rho_{0}^{2} \cdot 1-\frac{1}{2} \pi \cdot \rho_{i}^{2} \cdot I=\frac{1}{2} \pi \cdot\left(\rho_{0}+\rho_{i}\right) \cdot\left(\rho_{0}-\rho_{i}\right) \cdot 1=\pi \cdot R_{i} \cdot b \cdot I
$$

because $\rho_{0}+\rho_{i}=2 R_{i}$, where $R_{i}$ is the radial distance from the center of the vessel to the center of the segment and $\rho_{o}-\rho_{i}=b$, the segment width.

Based on the density $D_{i}$ (equation 2 ), the number of platelets present in the $i$-th annular semicylinder $\left(\mathrm{M}_{\mathrm{i}}\right)$ is defined by:

$$
M_{i}=D_{i} \cdot V_{R_{i}}=\pi \cdot \frac{N_{i} \cdot R_{i}}{f \cdot h}
$$

The total number of platelets in the vessel part as a whole, is the sum of the numbers of platelets (M) present in the 20 annular semicylinders:

$$
M=\sum_{i=1}^{20} M_{i}=\pi \sum_{i=1}^{20} \frac{N_{i} \cdot R_{i}}{f \cdot h}
$$

The total volume of the whole vessel part under consideration is $\pi . R^{2} .1$. The average platelet concentration in the vessel $(\overline{\mathrm{D}})$ is:

$$
\bar{D}=\frac{M}{\pi \cdot R^{2} \cdot I}=\frac{1}{R^{2} \cdot I \cdot f \cdot h} \cdot \sum_{i=1}^{20} N_{i} \cdot R_{i}
$$


The relative platelet concentration $\left(\mathrm{D}_{\mathrm{ret}}\right)$ in each segment becomes:

$$
D_{\text {xeI }}=\frac{D_{i}}{\bar{D}}=\frac{N_{i}}{\left(\frac{b}{R}\right) \cdot \sum_{i=1}^{20} N_{i} \cdot\left(\frac{R_{i}}{R}\right)}
$$

Note, that $R_{i} / R$ is the relative radial position $\left(0 \leq R_{i} / R \leq 1\right)$. Because 20 segments were chosen along the vessel diameter $20 \mathrm{~b}=2 \mathrm{R}$, and hence $b=0.1 R$.

\subsection{SUMMARY}

Platelet distribution was investigated in 21 venules (V) and 10 arterioles (A) of the rabbit mesentery (vessel diameter: 15-33 $\mu \mathrm{m}$ ). Circulating platelets were labelled in vivo with the dye acridine red and observed with fluorescence video microscopy. Only platelets flowing in a thin $(5-7 \mu \mathrm{m})$ optical section located about the median plane of the vessel were used. The relative position of each platelet, i.e. the distance of its centroid to the left vessel wall divided by the local vessel diameter, was determined. In addition, in 10 venules leukocyte margination was inhibited by intravenous injection of dextran sulfate (molecular weight: $500.000 ; 30$ $\mathrm{mg} / \mathrm{kg}$ bodyweight). The number of platelets per unit volume (i.e.,platelet density) relative to the mean density was significantly higher in the vessel center of $\mathrm{V}(1.04)$ than of $\mathrm{A}(0.55 ; \mathrm{p}<0.005)$. In contrast, near the wall this density was significantly higher in $A$ as compared to $V$. Mean values were near the wall (radial position $(R)=0.9-1.0$ ): 0.30 in $A$ and 0.11 in $V$ ( $p>0.05)$; at $R=0.8-0.9: 1.63$ in $A$ and 0.84 in $V(p<0.002)$; at $R=0.7-0.8$ : 1.60 in $A$ and 1.36 in $V(p>0.05)$; at $R=0.6-0.7: 1.16$ in $A$ and 1.60 in $V$ $(p<0.02)$; and at $R=0.5-0.6: 0.92$ in $A$ and 1.36 in $V(p<0.02)$. These differences in platelet distribution between arterioles and venules are not caused by the presence of leukocyte margination in venules. 


\section{INFLUENCE OF DEXTRANS ON PLATELET DISTRIBUTION IN ARTERIOLES AND VENULES}

\subsection{INTRODUCTION}

Platelet distribution differs greatly between arterioles and venules as observed in rabbit mesentery (Chapter 4; Woldhuis et al, 1992). In the vessel center, the relative platelet concentration is significantly lower in arterioles than in venules. Near the wall, the relative platelet concentration is higher in arterioles than in venules, while in the latter vessels a relative exclusion of platelets close to the vessel wall could be observed. The latter difference could not be explained by the presence of leukocyte margination in venules, because intravenous injection of a sulfated dextran with molecular weight 500,000 , a compound known to inhibit leukocyte rolling (Tangelder and Arfors, 1991), did not eliminate or reduce the relative platelet exclusion at the venular wall (Chapter 4; Woldhuis et al, 1992). Since it even tended to increase this wall exclusion of platelets, we also tested in some pilot experiments its effect on platelet distribution in arterioles. The findings suggested a considerable increase of the relative platelet concentration in the center of arterioles due to the dextran.

Dextrans bind to the surface of many cells, including platelets (Bygdeman and Eliasson, 1967; Langdell et al, 1958; Maupin, 1969), red blood cells (Chien, 1980) and endothelium (Bygdeman and Eliasson, 1967). A well known effect of the binding of high molecular weight dextrans to red blood cells is an increase in their tendency to aggregate (Chien, 1980; Palmer and Jedrzejczyk, 1975). Dextrans may also influence other rheological parameters, such as plasma viscosity and red blood cell deformability (Pfafferott et al, 1985). Because of our preliminary observations and these considerations, we investigated in the present study the effect of dextrans on platelet distribution in arterioles and venules. The results obtained with the above mentioned negatively charged dextran were

* in press, Eur J Physial 
compared with those obtained after injection of two neutral dextrans, one with the same and another with a ten times lower molecular weight. At the relatively low dose used, we also evaluated the effect of these three dextrans on plasma viscosity, red blood cell aggregation and red blood cell deformability. With regard to the effect of dextrans on these rheological parameters, it is of interest to know that most studies have been performed with human blood in vitro, and only few data are available in rabbits.

\subsection{MATERIALS AND METHODS}

A $500 \mathrm{kD}$ dextran charged negatively with sulfate groups (17\% of total weight; Dx500S) was obtained from Pharmacia Fine Chemicals (Uppsalla, Sweden). From Sigma Chemical Co (St. Louis, Mo., USA) we purchased two neutral dextrans with an average molecular weight of $485 \mathrm{kD}$ and 39 $\mathrm{kD}$, designated $\mathrm{D} \times 500$ and $\mathrm{Dx} 40$, respectively.

Experiments were performed on 2 groups of New Zealand white rabbits (see below). One group was used for intravital video microscopy and the other one for assessment of rheological parameters ex vivo. All dextrans were injected intravenously at a dose of $30 \mathrm{mg} / \mathrm{kg}$ bodyweight.

\section{Animals}

\section{Intravital microscopy group}

Twenty-five rabbits of either sex were divided into 4 subgroups of comparable weight (range 1.9-3.0 kg; mean $2.5 \mathrm{~kg}$ ): 6 control rabbits, 9 receiving Dx500S, 6 receiving Dx500 and 4 receiving Dx40. Anesthesia was induced by intramuscular injections of $40 \mathrm{mg} / \mathrm{kg}$ body weight ketamine hydrochloride (Nimatex, A.U.V., Cuijk, the Netherlands) and $4 \mathrm{mg} / \mathrm{kg}$ body weight xylazine hydrochloride (Sedamun, A.U.V.). Starting about 60 minutes after induction, anesthesia was maintained with the same substances (40 and $5 \mathrm{mg} / \mathrm{kg}$ bodyweight/hour, respectively), dissolved in a Lactetrol solution (Aesculaap, Boxtel, the Netherlands) and administered through a polyethylene catheter (PE 60) in the femoral vein $(15 \mathrm{ml} /$ hour).

Following induction of anesthesia, blood was collected from a central ear artery in disodium ethylenediamine tetraacetic acid (EDTA, 0.1 vol, $0.027 \mathrm{M}$ ). Platelets were counted twice with a Coulter Counter (model ZF). Hematocrit was measured in duplicate with an Autocrit II centrifuge (Clay Adams).

A PE-60 catheter was positioned in the femoral artery and kept patent 
by continuous perfusion with saline ( $5 \mathrm{ml} /$ hour) via an Intraflo II-system (Sorenson Research); no heparin was used. Mean arterial blood pressure and heart rate were measured with an external pressure transducer (CTC, $\mathrm{CP}-01$ ) and recorded on a physiological recorder (Schwarzer, US 266). Throughout the experiments mean blood pressure was $77 \pm 7 \mathrm{mmHg}$ (mean \pm standard deviation) and heart rate $146 \pm 29$ beats per minute. No significant differences between the 4 subgroups of rabbits existed (see subsection on statistics).

The trachea was cannulated and the cannula $(4.5 \mathrm{~mm}$ ID, Mallinckrodt) connected to an infant ventilator (Loosco, $\mathrm{mk} 2$ ). The rabbits were ventilated with a mixture of $91-94 \%$ air and $6-9 \%$ oxygen. Ventilation frequency was $60 /$ minute and tidal volume $15-20 \mathrm{ml}$, depending on the blood gas values. Blood gas and hemoglobin measurements were performed with an acid-base analyzer (ABL 3 Radiometer) in blood collected from a central ear artery 10 minutes after induction of ventilation. Mean values ( \pm S.D.) were $\mathrm{pH} 7.45 \pm 0.07, \mathrm{pCO}_{2} 34.9 \pm 6.3$ and $\mathrm{pO}_{2} 90.3 \pm 9.7 \mathrm{mmHg}$. No significant differences were found between the four subgroups.

\section{Hemorheology group}

Nineteen rabbits of either sex and with a mean weight of $2.7 \mathrm{~kg}$ (range: $2.2-3.8 \mathrm{~kg}$ ) were anesthetized as mentioned above. Blood samples were taken before, and 45 and 60 minutes after dextran injection, the time period during which platelet distribution was assessed in most experiments (range 15-90 minutes). Blood was collected from a femoral artery catheter: in EDTA for measurement of red blood cell deformability, hematocrit and hemoglobin concentration (OSM2 hemoximeter, Radiometer), and in heparinized tubes (Monoject, Sherwood Medicals, St. Louis, Mo., USA) for red blood cell aggregation and plasma viscosity. Ninety minutes after administration of the two neutral dextrans their plasma concentrations were determined (see subsection on assessment of rheological parameters).

\section{Intravital microscopy}

A short segment of the distal ileum was brought outside through a small abdominal midline incision. The mesentery was spread over a siliconized glass plate mounted on the stage of a Leitz intravital microscope adapted to telescopic imaging (Slaaf et al, 1982). It was continuously superfused with a buffered Tyrode solution at $37^{\circ} \mathrm{C}(\mathrm{pH} 7.37 \pm 0.04)$. The bowels were kept moist with overlying wet gauze.

To visualize the circulating blood platelets (see below), a Leitz SW 50x 
water-immersion objective (numerical aperture 1.0 ) and $2 \mathrm{X}$ projection eyepiece were employed. Fluorescence microscopy was performed with an incident illuminator (Leitz Ploemopak 2.2, tubefactor 1.6x) and interchangeable filter set N 2.1, (excitation filter: BP 515-560, dichroic mirror: RKP 580, barrier filter: LP 580). As light source a Xenon flash are fed by a Strobex power supply (Chadwick Helmuth) was used. The images were recorded through a high sensitivity TV-camera (Bosch TYC 9 A; one inch SIT tube, RCA $4804 \mathrm{HP}$ ) and stored on videotape (Betamax). Final optical magnification at the front plane of the TV camera was $200 \mathrm{X}$. To count the number of leukocytes rolling in a venule, transillumination and an $1 X$ projection eyepiece were used (final optical magnification $100 \mathrm{X}$ ). Vessel diameters were measured from video images using a home-built image shearing device.

Red blood cell velocities in microvessels were measured with a prism grating system (Slaaf et al, 1981), with the slit covering the whole vessel width. Actual mean velocities were obtained after division by 1.1. Velocities were measured prior to assessment of platelet distribution and before and after dextran injection. To obtain information about the mean local shear rate, we calculated reduced velocity (mean velocity divided by vessel diameter).

\section{Assessment of platelet concentration distribution}

Segments of venules and arterioles ranging in diameter from 17 to 33 $\mu \mathrm{m}$ were selected in such a way that no branch points and no adhering leukocytes could be observed upstream or downstream from the measuring site over a length of at least 10 vessel diameters. Experiments were performed at the focal position yielding the widest vessel diameter, i.e. in the median plane of the vessel.

To visualize the individual flowing platelets, $2 \mathrm{ml}$ of a solution of the dye acridine red $(7.5 \mathrm{mg} / \mathrm{ml})$ was injected through a polyethylene catheter positioned in a marginal ear vein (Tangelder et al, 1982a). With this procedure nearly all platelets are labelled without activation of the platelets by the dye or induction of gross ultrastructural changes. In addition, their capability to adhere and aggregate is maintained (Tangelder et al, 1982a). Leukocytes and the vessel wall are labelled as well, but red blood cells are not. One to 3 dye injections were given per animal, with an interval of 15-20 minutes between two injections. Following each injection the circulating platelets were recorded during 5 to 7 minutes by fluorescence video microscopy. 
Flashes of short duration $(<0.1 \mathrm{~ms})$ were given every 6 th TV field in the blanking period of the TV-camera. By using a flash of short duration, a smeared image due to platelet movement was prevented. With this flash interval we never detected a platelet, which had just entered the field of view $(39 \times 55 \mu \mathrm{m})$, in the next flashed image, i.e. $120 \mathrm{~ms}$ later.

To obtain a platelet distribution in a vessel, 150-160 still video fields were analyzed off-line, covering a time period of approximately 20 seconds. Since each flashed TV field represents an instantaneous picture, showing the position of none, one or more platelets at that moment, a possible influence of differences in platelet velocity can be neglected.

Only platelets flowing within a thin optical section were used for analysis (Tangelder et al, 1982b). Its depth of about $5 \mu \mathrm{m}$ is constant within an experiment over the full length and width of the vessel part under observation (Tangelder et al, 1985). The video fields were digitized on an IBM personal computer ( 512 by 512 pixels; intensity range $0-255$ ). A platelet was considered to be within the optical section when the intensity peak of a line of 3 pixels wide and drawn through the center of its image and the vessel lumen exceeded the background noise.

On a transparency positioned over the monitor screen we drew the vessel walls and marked within a segment with a length of approximately 27 $\mu \mathrm{m}$ the centers of gravity (centroids) of platelet images. The distance (d) between the platelet centroid and left vessel wall (according to flow direction), as well as the vessel diameter at that specific point $(2 \mathrm{R})$, were determined and the relative position of the platelet $(\mathrm{d} / 2 \mathrm{R})$ calculated. The number of platelets counted during analysis ranged from 52 to 256 (mean $119 \pm 46$ ) in arterioles and from 55 to 297 (mean $139 \pm 61$ ) in venules, with no significant differences between the four subgroups.

For each vessel a frequency distribution was obtained by grouping the platelet counts into 20 equal segments across its diameter. Individual distributions were normalized with respect to the mean concentration over the whole cross-sectional area, and not for the mean within the optical section. This is necessary for proper comparison of the vessels in the different subgroups. Although the 20 segments within the optical section are of equal volume, they contribute to the mean platelet concentration in a vessel to a different extent. The calculation of the mean platelet concentration has been described in detail elsewhere (Chapter 4; Woldhuis et al, 1992). In short, the relative platelet concentration in a particular segment $\left(D_{\text {rel }, i}\right)$ is given by: 


$$
D_{x \in I_{1}}=\frac{N_{1}}{D_{\text {mean }}}=\frac{N_{i}}{\sum_{i=1}^{20} N_{i} \cdot\left(\frac{\pi b R_{i}}{\pi R^{2}}\right)}=\frac{N_{i}}{\left(\frac{b}{R}\right) \cdot \sum_{i=1}^{20} N_{i} *\left(\frac{R_{i}}{R}\right)},
$$

where, $\quad N_{i}=$ number of platelets counted in $i$-th segment

$$
\begin{aligned}
& \mathrm{D}_{\text {mean }}=\text { mean platelet concentration over the whole vessel cross-section } \\
& \mathbf{R} \quad \text { = distance from vessel center to center of } i \text {-th segment } \\
& \pi \mathrm{R}^{2} \quad \text { surface area of the whole vessel cross-section } \\
& \mathrm{R}=\text { vessel radius } \\
& \pi \mathrm{bR}_{\mathrm{i}}=\text { surface area of the } \mathrm{i} \text {-th segment } \\
& \mathrm{b} \quad=\text { width of each of the segments }
\end{aligned}
$$

Due to the finite depth of the optical section, the distance from a platelet to the vessel center may be longer than the observed distance. This holds especially for platelets observed in the central segments. Because the actual depth of the optical section in each individual experiment was not known, we present distributions as measured. However, for statistical testing of effects occurring in the vessel center, we always used the mean of the 4 central segments $(0.0-0.4 \mathrm{R})$, thereby largely reducing the influence of this error. Moreover, because the contribution of these 4 central segments to the total cross-sectional area of the vessel is only $16 \%$, the influence of these segments on the calculation of the mean concentration (see above), is also limited.

To bring out possible differences between the different groups more clearly, the left and right hand sides of a vessel were pooled. In our previous study (Chapter 4; Woldhuis et al, 1992), the coefficients of variation in the 20 segments of the general distribution did not differ much, both in arterioles and venules, indicating rather symmetric individual distributions. Moreover, the flow conditions in straight, unbranched parts of a microvessel are in general cylindrically symmetric, because of the short entrance length in vessels of this size (Caro, 1978). In addition, individual histograms were pooled, which is justified by the observation that vessel diameter and systemic platelet count do not significantly influence platelet distribution in rabbit mesenteric arterioles or venules (Chapter 4; Woldhuis et al, 1992).

\section{Assessment of theological parameters}

Red blood cell aggregation was determined in a cone-plate aggregometer (Myrenne GMbH, Aachen, Germany), using the M1-mode (rotational 
shear of $600 \mathrm{~s}^{-1}$ for 10 seconds, followed by $5 \mathrm{~s}^{-1}$ for 3 seconds). The transmission of infrared light is measured with photosensors during aggregate formation and expressed in arbitrary units. On each sample 5 measurements were performed within 15 minutes after blood collection. The mean value was taken of the 3 most centrally located values.

Red blood cell deformability was measured with a Laser assisted Optical Rotational Deformability meter (Hardeman and Rie, 1991). A laser beam is passed through an erythrocyte suspension, present in a gap between an inner stationary cylinder and an outer rotating cylinder. The diffraction pattern is projected onto a screen and detected by a videocamera, which is linked to a personal computer. The erythrocyte elongation index (EI) is then calculated from the diffraction pattern by measuring the lengths of its major (A) and minor (B) axes $[\mathrm{EI}=(\mathrm{A}-\mathrm{B}) /(\mathrm{A}+\mathrm{B})]$. Polyvinylpyrrolidone (PVP) and DX500 $(144 \mathrm{mg} / \mathrm{ml}$ physiological saline) were used as suspending medium, both having the same osmolality $(290 \mathrm{mOsmol} / \mathrm{kg})$ and viscosity (about $45 \mathrm{mPa} . \mathrm{s}$ ). In all experiments blood was diluted $10 \mathrm{x}$ in the suspending medium.

Plasma viscosity was measured with a capillary viscometer at $37^{\circ} \mathrm{C}$ (Rheomed GMbH, Aachen, Germany). At a constant driving pressure, a plasma sample flows through a polyethylene tube with an inner diameter of $800 \mu \mathrm{m}$. From the flow velocity the viscosity is calculated according to Poiseuille's law. Two measurements were performed on each plasma sample and the mean value was taken.

Dextran concentrations were determined in heparinized plasma. Dextran consists of glucose units. The dextran, but also glycoproteins in the plasma sample, were hydrolysed into monosaccharide moieties in $1 \mathrm{~N} \mathrm{HCl}$ during 3 hours at $100^{\circ} \mathrm{C}$. Glucose levels were then determined with the otoluidine method (Hultman, 1974), in both a boiled and non-boiled plasma sample. However, this method also detects fructose. To correct for glucose and fructose moieties hydrolysed from glycoproteins, we also applied the otoluidine method to boiled and non-boiled control plasma, i.e. before dextran injection. For sulfated dextrans this method cannot be used, and, hence, we did not determine their concentration. For all tested parameters, values obtained at 45 minutes did not differ significantly from those obtained at 60 minutes, and hence these two values were pooled for each experiment.

\section{Statistical analysis}

The 95\% confidence limits of the pooled distributions as presented 
in Figures 5.1 to 5.3, were calculated using the standard error of the mean. All other parameters are presented as ranges and/or means with standard deviations. Differences between groups and vessels were tested with the Kruskal-Wallis one way analysis of variance. Two independent groups were compared with the Mann-Whitney $U$ test. In case of paired data-groups the Wilcoxon signed-rank test was used. When the p-value was equal to or exceeding 0.05 , a difference was considered statistically non-significant. Correlations between two sets of data were tested with the Spearman rank correlation test. SPSS-PC statistical package (SPSS Inc., Chicago, USA) was used in all statistical tests.

\subsection{RESULTS}

The systemic hematocrit values of all animals ranged from 34 to $42 \%$ (mean $38 \pm 2 \%$ ), with no statistical differences between the various groups. The same holds for the systemic platelet counts (range 207-582x 10 $/ 1$; mean $382 \pm 104)$. In case of the hemoglobin concentration, a significant difference was found between the intravital microscopy group $(8.5 \pm 0.9 \mathrm{mmol} / \mathrm{l})$ and the hemorheology group $(7.3 \pm 0.6 \mathrm{mmol} / \mathrm{l})$. Subgroups within these two groups did not differ. The difference in hemoglobin content between both groups is partly due to the different methods used (ABL 3 versus OSM 2). In a separate set of experiments, nine blood samples were studied with both methods. The ABL showed a significantly higher $(p<0.01)$ hemoglobin content than the OSM2 $(7.4 \pm 0.5$ and $7.1 \pm 0.4 \mathrm{mmol} / 1$, respectively). The hematocrits, platelet counts and hemoglobin contents found are within normal ranges for rabbits (Kozma, 1974). Ninety minutes after dextran injection, its concentration in the plasma of 4 rabbits was $0.1,0.4,0.4$ and $0.5 \mathrm{mg} / \mathrm{ml}$ for Dx40 and $0.3,0.8,1.0$ and $1.1 \mathrm{mg} / \mathrm{ml}$ for Dx500.

\section{Platelet distributions}

The influence of sulfated dextran (Dx500S) on platelet distribution is presented in Figure 5.1. In arterioles, Dx500S increased the relative platelet concentration in the center of the vessel and decreased it close to the wall (Figures 5.1A and B). Both in the center $(0.0-0.4 \mathrm{R})$ and near the wall $(0.8-$ $0.9 \mathrm{R}$ ) of arterioles the changes induced by sulfated dextran were statistically significant ( $p<0.003$ and $p<0.03$, respectively). Comparison of Figures $5.1 \mathrm{C}$ and 5.1D shows in venules a similar, but less pronounced and non-significant effect of the sulfated dextran. 


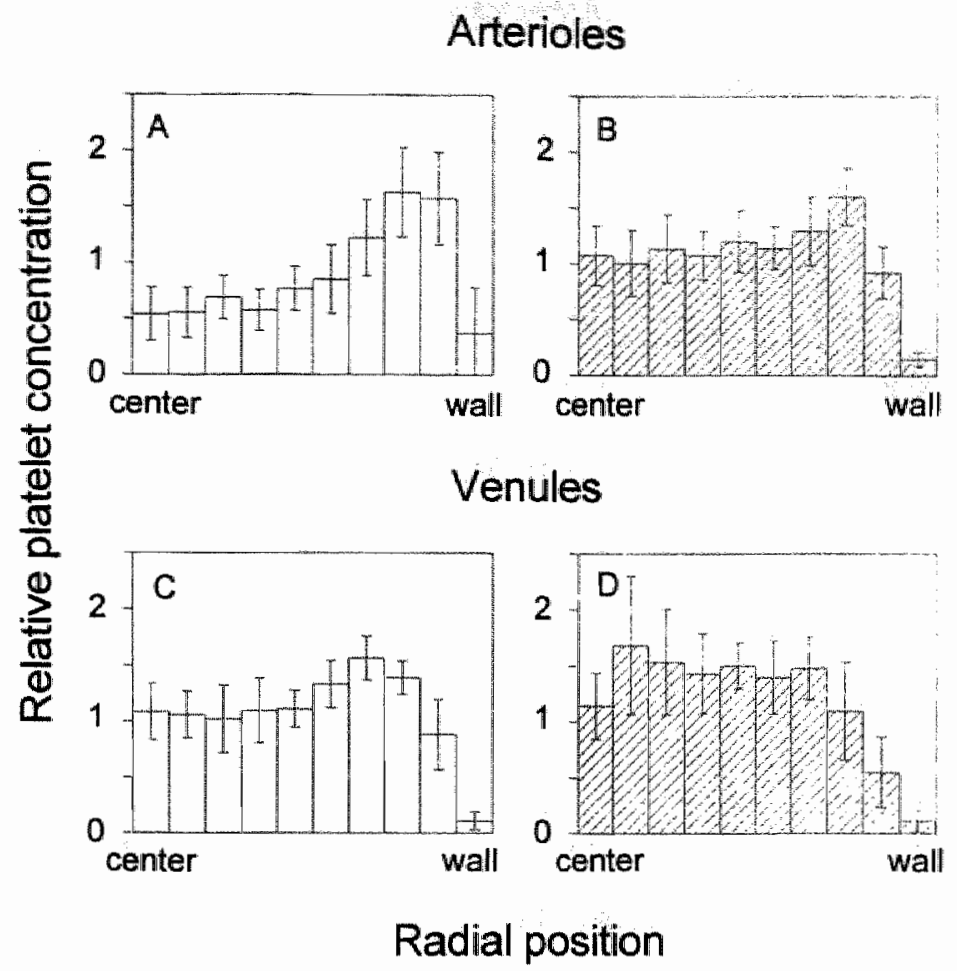

Figure 5.1: Influence of a $500 \mathrm{kD}$ dextran sulfate (Dx500S) on the relative platelet concentration distribution in arterioles $(B)$ and venules $(D)$, as compared to control distribution ( $A$ and $C$, respectively). The mean values and $95 \%$ confidence limits are presented. Each individual distribution was normalized with respect to the mean concentration over the whole crass-sectional area of that vessel. Number of vessels: $A=9, B=10, C=10$ and $D=10$.

In arterioles, neutral dextran of the same molecular weight (Dx500) showed a similar significant effect (Figures 5.2A and B), both in the center $(0.0-0.4 \mathrm{R} ; \mathrm{p}<0.001)$ and near the wall $(0.8-0.9 \mathrm{R} ; \mathrm{p}<0.02)$. Hence, the effect of sulfated dextran on platelet distribution in arterioles was not due to its negative charge. In venules, this neutral $500 \mathrm{kD}$ dextran also induced a slight, but non-significant, increase of the relative platelet concentration in the vessel center and a decrease near the wall (Figures 5.2C and D).

The neutral $40 \mathrm{kD}$ dextran tended to influence the platelet distribution in arterioles (Figures 5.3A and $\mathrm{B}$ ) in the same direction as the other two dextrans (Dx500 and Dx500S). These changes, however, did not reach the level of significance, as tested for segments $0.0-0.4 \mathrm{R}$ and $0.8-0.9 \mathrm{R}$. 


\section{Arterioles}

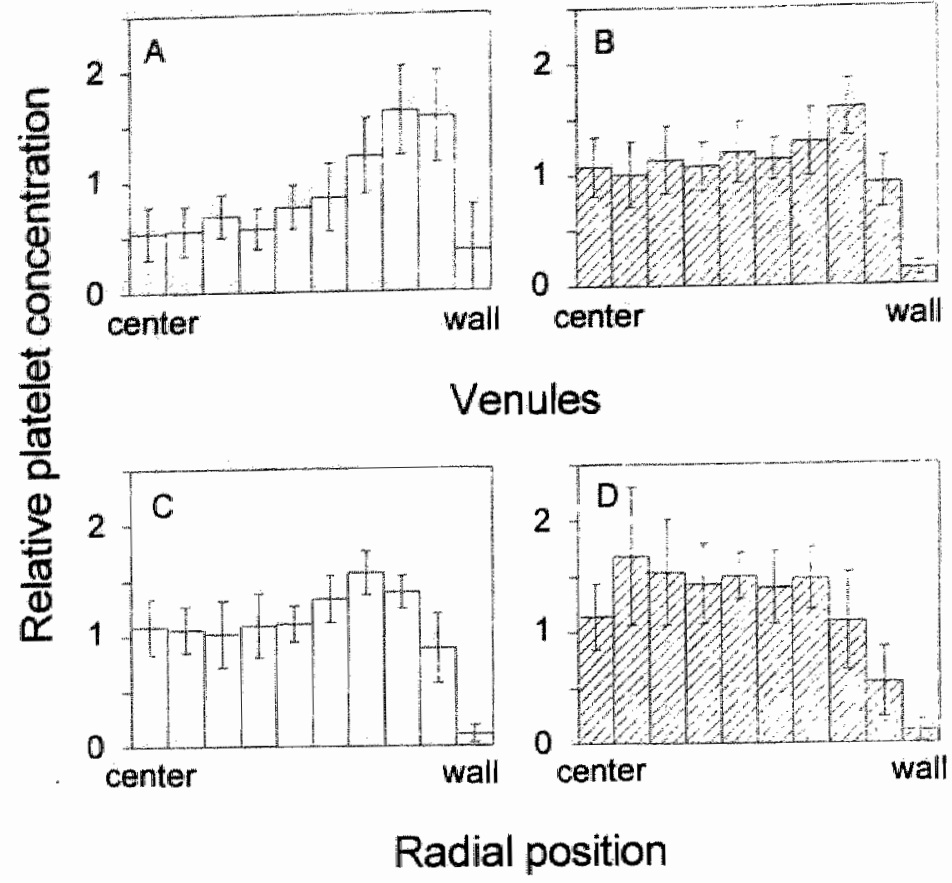

Figure 5.2: Influence of a neutral $500 \mathrm{kD}$ dextran (Dx500) on the relative platelet concentration distribution in arterioles $(B)$ and venules $(D)$, as compared to control distribution ( $A$ and $C$, respectively). The mean values and $95 \%$ confidence limits are presented. Each individual distribution was nornalized with respect to the mean concentration over the whole cross-sectional area of that vessel. Number of vessels: $A=9, B=10$, $C=10$ and $D=10$.

In venules, on the contrary, this dextran unexpectedly decreased the platelet concentration in the center of the vessel $(0.0-0.4 \mathrm{R} ; \mathrm{p}<0.03)$ and increased it near the wall (0.8-0.9R: $p<0.04$; Figures 5.3C and D).

Comparison between the arterioles and venules within each dextran group (Figures 5.1B and D, 5.2B and D, and 5.3B and D, respectively), revealed no significant differences for the segments $0.0-0.4 \mathrm{R}$. By contrast, in the control group the relative platelet concentration in the segments 0.0 $0.4 \mathbb{R}$ was higher in venules than in arterioles $(p<0.04)$. For segment 0.8 $0.9 \mathrm{R}$, a significant difference between arterioles and venules was found in the control, Dx500S and Dx500 groups $(\mathrm{p}<0.006, \mathrm{p}<0.02$ and $\mathrm{p}<0.05$, respectively), but not in the Dx40 group. 


\section{Arterioles}
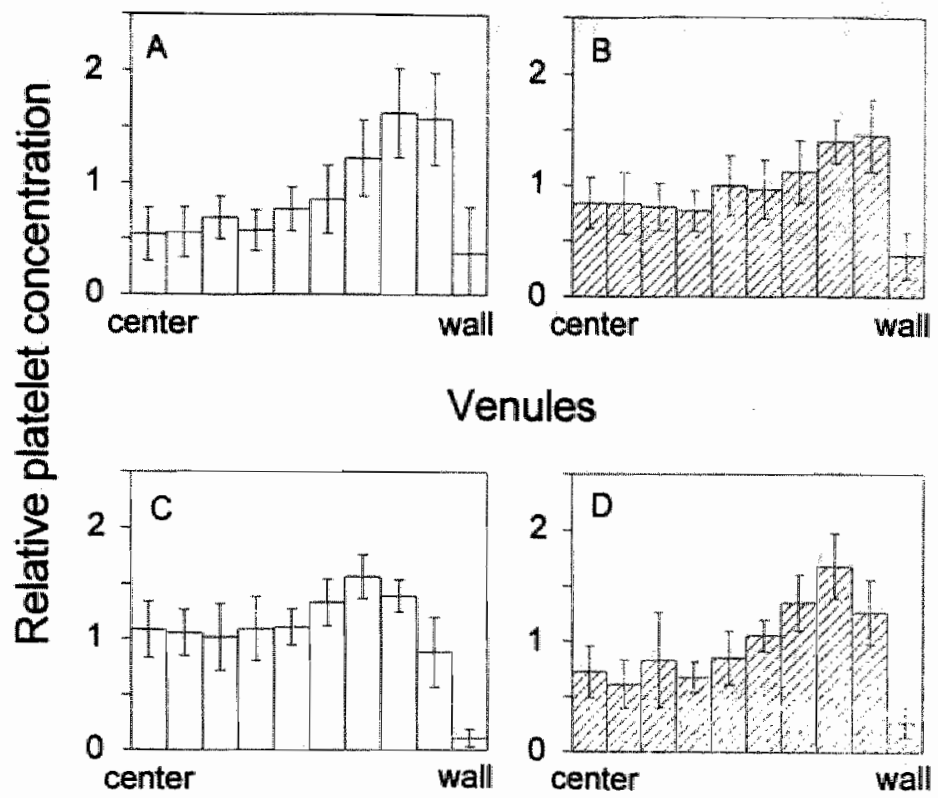

Venules

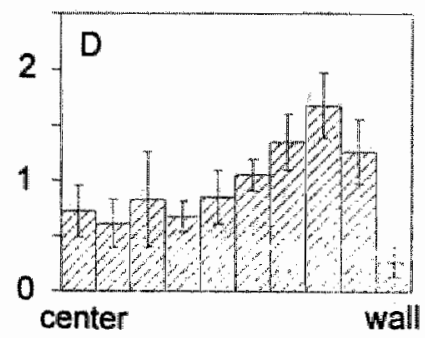

Radial position

Figure 5.3: Influence of a neutral $40 \mathrm{kD}$ dextran $(D \times 40)$ on the relative platelet concentration distribution in arterioles $(B)$ and venules $(D)$, as compared to control distribution $(A$ and $C$, respectively). The mean values and $95 \%$ confidence limits are presented. Each individual distribution was normalized with respect to the mean concentration over the whole crosssectional area of that vessel. Number of vessels: $A=9, B=10, C=10$ and $D=10$.

\section{Microvascular parameters}

Vascular diameters ranged from 17 to $33 \mu \mathrm{m}$, with a mean value in the 39 arterioles used of $23.5 \mu \mathrm{m}( \pm 4.2 \mu \mathrm{m})$ and in the 38 venules of 24.2 $\mu \mathrm{m}( \pm 4.1 \mu \mathrm{m})$. No differences in diameter existed between the various subgroups.

Table 5.1 presents the red blood cell velocities measured prior to assessment of the platelet distribution in the Dx40 and Dx500 groups, as well as the calculated reduced velocities, which are a measure of mean local shear rate. No differences were found between the DX40 and DX500 groups. However, in Dx40 venules the velocity and U-values were significantly lower than in DX40 arterioles. 
Table 5.1: Mean red blood cell velocity and reduced velocity (U) prior to assessment of platelet distribution in arterioles and venules ( $n=$ number of vessels) of $D \times 40$ and $D \times 500$ treated animals. Mean values and standard deviations are given.

\begin{tabular}{|c|c|c|c|c|c|c|}
\hline \multicolumn{4}{|c|}{ Artterioles } & \multicolumn{3}{|c|}{ Venules } \\
\hline group & n & $\begin{array}{l}\text { velocity } \\
(\mathrm{mm} / \mathrm{s})\end{array}$ & $\underset{\left(s^{-1 i}\right)}{\mathbb{U}}$ & $\mathrm{n}$ & $\begin{array}{l}\text { velocity } \\
(\mathrm{mm} / \mathrm{s})\end{array}$ & $\begin{array}{c}U \\
\left(s^{-1}\right)\end{array}$ \\
\hline Dx40 & 9 & $3.4 \pm 1.7$ & $130 \pm 67$ & 7 & $1.5 \pm 0.9^{*}$ & $56 \pm 37$ * \\
\hline D*500 & 9 & $2.2 \pm 1.4$ & $84 \pm 56$ & 9 & $2.2 \pm 0.9$ & $82 \pm 33$ \\
\hline
\end{tabular}

* $=p<0.05$ compared to DX40 arterioles

Injection of dextrans did not cause changes in red blood cell velocity (Table 5.2) or diameter and, hence, in reduced velocity, an indicator of mean local shear rate. No correlations could be found between the mean value of the relative platelet concentration in the 4 central segments and reduced velocity. In addition, for none of the dextrans was there a correlation between the time after injection that platelet distribution was assessed, and the mean platelet concentration in the 4 central segments.

Leukocyte rolling was never seen in arterioles, but was generally present in venules, with no significant differences between the 4 groups (Table 5.2, column before dextran). Injection of $\mathrm{Dx} 40$ had no influence, Dx500 caused a decrease of about $23 \%$, while dextran sulfate inhibited leukocyte rolling almost completely (Table 5.2). Comparison of the groups after dextran injection showed no difference between Dx40 and Dx500, while the sulfated dextran group differed significantly from the other two.

Injection of acridine red had no significant influence on leukocyte rolling, the mean values before and after injection being $48 \pm 20$ and $46 \pm$ 29 cells/minute $(n=7)$, respectively.

\section{Hemorheology}

As tested in a separate group of animals, none of the dextrans had a significant effect on hematocrit or hemoglobin concentration. Dx40 or DX500 did not influence red blood cell deformability (Figure 5.4). The elongation indices of blood containing DX40 or Dx500 after an intravenous injection of $30 \mathrm{mg} / \mathrm{kg}$ bodyweight did not differ from control blood. Even if polyvinylpyrrolidone as suspending medium was replaced by a Dx500 solution with the same osmolality and viscosity no difference in elongation index could be detected. 
Table 5.2: Influence of dextran $(30 \mathrm{mg} / \mathrm{kg})$ on red blood cell velocity and leukocyte rolling: Values measured prior to and 3-5 minutes after introvenous injection $(n=$ number of animals; one vessel per animal). Mean values and standard deviation are given.

\begin{tabular}{|c|c|c|c|c|}
\hline \multicolumn{2}{|r|}{ Arterioles } & & & Venules \\
\hline $\mathbf{n}$ & before after & n & before & after \\
\hline
\end{tabular}

Red blood cell velocity $(\mathrm{mm} / \mathrm{s})$

\begin{tabular}{lllllll} 
Dx40 & 4 & $2.4 \pm 0.4$ & $2.6 \pm 0.2$ & 4 & $1.5 \pm 0.6$ & $1.6 \pm 0.9$ \\
Dx500 & 4 & $3.1 \pm 0.7$ & $3.2 \pm 0.8$ & 5 & $2.4 \pm 0.6$ & $2.6 \pm 0.6$ \\
\hline \hline
\end{tabular}

Leukacyte rolling per minute

\begin{tabular}{llllllc} 
control & 6 & 0 & 0 & 6 & $49 \pm 20$ & $\cdots$ \\
Dx40 & 4 & 0 & 0 & 4 & $48 \pm 20$ & $45 \pm 20$ \\
Dx500 & 6 & 0 & 0 & 6 & $39 \pm 7$ & $30 \pm 10^{*}$ \\
Dx500S & 9 & 0 & 0 & 9 & $54 \pm 32$ & $0-1$ \\
\hline
\end{tabular}

* $=\mathrm{p}<0.05$ compared to before, ${ }^{* *}=$ zero in all, except two

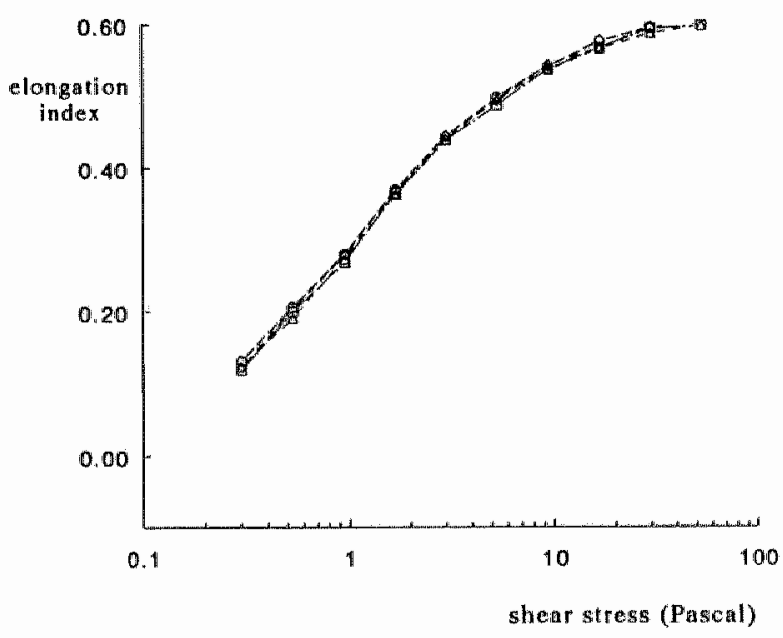

Figure 5.4: Deformability of red blood cells in the presence and absence of dextrans. Diamonds and triangles is control blood suspended in polyvinylpyrrolidone (PVP) or in Dextran $500(144 \mathrm{mg} / \mathrm{ml}$ physiological saline), respectively; both solutions having an osmolality of $290 \mathrm{mOsmol} / \mathrm{kg}$ and a viscosity of about $45 \mathrm{mPas}$. Circles and squares are blood samples collected from a rabbit 15 minutes after injection of $30 \mathrm{mg} / \mathrm{kg}$ of Dx40 or Dx500, respectively; these samples were suspended in PVP. 
Table 5.3: Influence of dextran $(30 \mathrm{mg} / \mathrm{kg} \mathrm{V})$ or physiological saline on red blood cell (RBC) aggregation (arbitrary units) and plasma viscosity (mPas). Means and standard deviations are presented.

\begin{tabular}{llllll}
\hline & Animals & \multicolumn{2}{c}{ RBC aggregation } & \multicolumn{2}{c}{ Plasma viscosity } \\
& & before & after & before & after \\
\hline SALINE & 3 & $1.1 \pm 0.6$ & $1.2 \pm 0.8$ & $1.09 \pm 0.02$ & $1.10 \pm 0.01$ \\
DX40 & 4 & $1.6 \pm 0.9$ & $1.8 \pm 1.2$ & $1.23 \pm 0.09$ & $1.25 \pm 0.10$ \\
Dx500 & 6 & $0.5 \pm 0.4$ & $1.2 \pm 0.7 *$ & $1.05 \pm 0.07$ & $1.09 \pm 0.06$ \\
Dx500S & 6 & $0.7 \pm 0.5$ & $1.5 \pm 0.8 *$ & $1.15 \pm 0.11$ & $1.17 \pm 0.09$ \\
\hline
\end{tabular}

* = $\mathrm{p}<0.05$ compared to before

The influence of dextrans on red blood cell aggregation is presented in Table 5.3. Each of the two high molecular weight dextrans increased red blood cell aggregation approximately twofold, while Dx40 or saline had no significant effect. Table 5.3 also shows that an intravenous injection of 30 $\mathrm{mg} / \mathrm{kg}$ bodyweight of either of the dextrans did not significantly change plasma viscosity.

\subsection{DISCUSSION}

The present study shows that intravenous injection of a relatively low dose of neutral or sulfated high molecular weight dextran $(500 \mathrm{kD})$ increases the platelet concentration in the center of arterioles and decreases it near their wall. A similar but non-significant effect was observed in venules. Injection of a neutral $40 \mathrm{kD}$ dextran, however, had little or no effect in arterioles, but decreased the platelet concentration in the center of venules and increased it near their wall. These changes in platelet distribution could not be explained by differences in flow velocity. Levels of leukocyte margination in venules had no effect on platelet distribution in these vessels. In contrast to Dx40, the high molecular weight dextrans increased the red blood cell aggregation tendency. Neither substance influenced red blood cell deformability or plasma viscosity.

The dextran dose of $30 \mathrm{mg} / \mathrm{kg}$ bodyweight injected in this study is low as compared to the doses used for hemodilution, i.e. $1000-2000 \mathrm{mg} / \mathrm{kg}$ bodyweight (Engeset et al, 1967). It can be easily calculated that changes in osmolality caused by this low dose of dextran are negligibly small. Moreover, no changes in systemic hematocrit were observed, indicating that plasma 
volume did not expand significantly. No increases in mean arterial blood pressure or changes in microvascular red blood cell velocity were seen. Therefore, it is unlikely that the changes in platelet distribution caused by the dextrans are due to hemodilution or plasma expansion.

The plasma concentration of dextran shortly after injection will have been on the average approximately $0.8-0.9 \mathrm{mg} / \mathrm{ml}$, assuming in rabbits a mean blood volume of $56 \mathrm{ml} / \mathrm{kg}$ bodyweight (Kozma, 1974) and a mean hematocrit of $38 \%$ (this study). The plasma concentration of $500 \mathrm{kD}$ dextran found 90 minutes after injection therefore indicates little disappearance of this dextran during this period, which is in agreement with findings reported in dogs (Bryant et al, 1964). By contrast, about half of the injected $40 \mathrm{kD}$ dextran was cleared from the plasma within this period of time. A comparable figure of about $40 \%$ has been reported in humans (Gruber, 1969). It is unlikely that the absence of an effect on platelet distribution in arterioles, as observed after injection of the $40 \mathrm{kD}$ dextran, is caused by its relatively rapid clearance from the circulation, because this dextran did show a significant effect on platelet distribution in venules. In addition, in both vessel types no correlation was found between platelet distribution and time after injection of this dextran.

The high molecular weight dextrans increased platelet concentration in the center of arterioles and decreased it near the wall. Dextran injection did not change microvascular flow velocity. However, it cannot be excluded that the shape of the velocity profile changed under influence of these dextrans. In mesenteric arterioles the velocity profile is blunted (Tangelder et al, 1986). In case of a change in shape due to dextran, one may expect a higher degree of blunting (Rosenblum, 1972), leading to an increase in wall shear rate. It seems unlikely, however, that such an increase in wall shear rate can be held responsible for the increase in platelet concentration in the center of arterioles following dextran injection. The findings in the in vitro study of Tilles and Eckstein (1987) indicate that the platelet distribution is independent of wall shear rate at values higher than $430 \mathrm{~s}^{-1}$. Generally, higher wall shear rates are found in arterioles in vivo (Tangelder et al, 1988).

Because dextran injection did not change microvascular flow velocity and plasma viscosity, the effects on platelet distribution as observed in this study are probably caused by binding of the high molecular weight dextran to the surface of platelets (Bygdeman and Eliasson, 1967; Langdell et al, 1958; Maupin, 1969) and/or red blood cells (Chien, 1980). High molecular weight dextrans may induce platelet aggregation, partially by acting as macromolecular bridges between cells (Bygdeman and Eliasson, 1967). 
However, in none of our experiments did we observe platelet doublets, triplets or larger aggregates, making platelet aggregation an unlikely cause for the observed changes in their distribution.

A second possibility is that the high molecular weight dextrans acted as bridging molecules between platelets and red blood cells. By such a mechanism red blood cells might trap platelets in the center of the vessel. Red blood cells tend to migrate towards the vessel center (Goldsmith, 1972; Palmer and Jedrzejczyk 1975). It is usually assumed that central crowding of red blood cells disperse the lighter and less deformable platelets from the center of a vessel (Goldsmith, 1972; Palmer, 1967; Tilles and Eckstein, 1987), an effect observed in arterioles in vivo (this study, Tangelder et al, 1985; Woldhuis et al, 1992), and surmised in small arteries (Ofjord and Clausen, 1986). Formation of multiple macromolecular bonds between platelets and red blood cells, albeit transient, may decrease the platelet dispersion from the vessel center and trap platelets in the red blood cell rich core.

Beside by a direct interaction with platelets, dextrans may influence their distribution by changing rheological parameters. Red blood cell deformability and plasma viscosity both influence the tendency of red blood cells to migrate towards the center of the vessel (Goldsmith, 1972; Palmer and Jedrzejczyk, 1975). Since these two factors were not influenced by high molecular weight dextran, they can probably be excluded as a cause for its effect on platelet distribution.

Another rheological factor which could play a role is the tendency of erythrocytes to form aggregates (Palmer, 1967). At the dose used, the high molecular weight dextrans increased red blood cell aggregation tendency approximately twofold. Although this influence on red cell aggregation was inferred from measurements in vitro in simple shear flow, it is possible that in vivo an increased number of macromolecular bridges between red blood cells hampered their capability to disperse platelets from the vessel center. With regard to the changes in platelet distribution observed in small arterioles, one should realize that an effect of the dextran on cell behavior in larger feeding vessels and at upstream bifurcations, might be responsible for the changes in platelet distribution observed downstream. However, which of the above mentioned explanations is valid, remains subject to further investigation.

Our observation of an increased platelet concentration in the center of arterioles due to high molecular weight dextran, is in contrast to its influence on white blood cell distribution in small tubes in vitro (Nobis, 1985; Palmer, 1967). Induction of strong red blood cell aggregation resulted 
in an enhanced white blood cell margination, especially at lower shear values. However, these studies used an approximately tenfold higher dextran concentration.

Near the vessel wall, platelet concentration decreased after injection of one of the $500 \mathrm{kD}$ dextrans, especially in arterioles. This might have been caused by the greater access of platelets to occupy the vessel center. By contrast, the $40 \mathrm{kD}$ dextran significantly increased the platelet concentration near the wall in venules, but not in arterioles. In venules, but not in arterioles, there seems to be a relative exclusion of platelets close to the wall (Woldhuis et al, 1992), presumably due to differences in endothelial glycocalyx composition and surface charge. Dextrans also bind to endothelium (Bygdeman and Eliasson, 1967). This might shield structures involved in the exclusion of platelets at the wall. The reason that shielding of endothelial structures by the high molecular weight dextrans, if any, did not increase the platelet concentration near the venular wall, in contrast to the $40 \mathrm{kD}$ dextran, might be explained by the finding that they induced an increase in platelet concentration in the vessel center, thereby cancelling any effect due to shielding of vessel wall structures.

It is unknown to which extent the platelet distribution in a microvessel influences hemostasis. The time needed to form a hemostatic plug is much shorter in mesenteric arterioles than venules (Bergqvist and Arfors, 1976). It is conceivable that the observed difference in platelet distribution between arterioles and venules plays a role. It has been reported, for example, that high molecular weight dextrans produce a more severe disturbance of bleeding time than low molecular weight dextran, although the doses used were high (Langdell et al, 1958). Low molecular weight dextrans do not significantly influence hemostatic plug formation time in mesenteric microvessels (Bergqvist and Arfors, 1976). Our present finding that a relatively low dose of a neutral high molecular weight dextran clearly influences the platelet distribution in mesenteric arterioles, allows to study in future experiments the importance of the platelet distribution in microvessels for hemostasis.

In conclusion, a low dose of a high molecular weight dextran significantly increased the platelet concentration in the vessel center of arterioles, irrespective of their charge. Low molecular weight dextran had no such effect, but increased in venules the platelet concentration near the vessel wall. These effects are probably explained by the binding of dextrans to the surface of platelets and/or red blood cells and/or endothelium. 


\subsection{SUMMARY}

Dextrans bind to the surface of platelets, red blood cells and endothelium. We investigated whether a low dose $(30 \mathrm{mg} / \mathrm{kg}$ IV) of a $40 \mathrm{kD}$ (Dx40), neutral $500 \mathrm{kD}$ (Dx500) or sulfated $500 \mathrm{kD}$ (Dx500S) dextran influences platelet distribution in rabbit mesenteric arterioles and venules (diameter 17-33 $\mu \mathrm{m}$ ). Intravital fluorescence videomicroscopy was used to visualize platelets labelled in vivo with acridine red. Their concentration distribution as determined within a thin optical section about the median vessel plane was expressed relative to the mean concentration in that vessel. In arterioles, Dx500 and Dx500S increased in the center (radial position (R): $0.0-0.4 R$ ) the relative platelet concentration from 0.60 to 1.07 $(\mathrm{p}<0.001)$ and to $1.20(\mathrm{p}<0.003)$, and near the wall $(0.8-0.9 \mathrm{R})$ reduced it from 1.59 to $0.93(\mathrm{p}<0.02)$ and to $0.95(\mathrm{p}<0.03)$, respectively. In venules a similar but non-significant effect was observed. Dx 40 did not change platelet distribution in arterioles, but in venules their concentration decreased in the center from 1.08 to $0.71(\mathrm{p}<0.03)$ and increased at the wall from 0.89 to 1.27 ( $p<0.04$ ). Deformability of red blood cells was unchanged, but their aggregation tendency increased approximately twofold after Dx500 and Dx500S injection, while Dx40 had no influence. Leukocyte margination in venules did not affect platelet distribution. Dextran injection did not change microvascular flow velocity or plasma viscosity, suggesting that the observed changes in arteriolar platelet distribution were caused by binding of dextran to the surface of platelets and/or red blood cells.

\section{Acknowledgement}

The authors wish to thank Dr M.R. Hardeman, Dept. of Internal Medicine and Clinical Chemistry, University Hospital, Academic Medical Centre, Amsterdam, for permission to use the Laser assisted Optical Rotational Deformability meter. 


\section{VELOCITY PROFILES IN RABBIT MESENTERIC VENULES IN VIVO}

\subsection{INTRODUCTION}

Interactions between blood cells, plasma components and the vessel wall depend among others on the distribution of shear rates or velocity gradients in a vessel (Goldsmith and Turitto, 1986; Turitto, 1982). Precise measurements of the velocity profiles in microvessels are difficult to perform. In vitro studies have yielded conflicting results and have not been performed in glass tubes smaller than $30 \mu \mathrm{m}$ (Bugliarello and Hayden, 1963; Bugliarello and Sevilla, 1970; Goldsmith, 1972; Baker and Wayland, 1974; Goldsmith and Marlow, 1979). Recently, we have been able to accurately determine for the first time the shape of the velocity profile in arterioles smaller than $33 \mu \mathrm{m}$ (Tangelder et al, 1986). The circulating blood platelets were fluorescently labelled in vivo and used as natural markers of flow. The study showed that blood flow in small arterioles (diameter 17-32 $\mu \mathrm{m}$ ) cannot be described with a one-fluid model, i.e. uniform flow properties over the whole vessel cross-section. A thin layer of fluid at the wall has a considerably lower apparent viscosity than the fluid in the remainder of the vessel. This causes relatively high velocities near the vessel wall, resulting in a blunting of the profile. However, also the shape of the velocity profile in the remainder of the vessel was significantly flattened as compared to a parabola. From these profiles we also obtained least estimates of wall shear rate (Tangelder et al, 1988).

In venules, however, detailed knowledge of the velocity profile is still lacking. Extrapolation from data obtained in arterioles or in vitro is not without risk, because venules differ from arterioles in many aspects. They differ in flow pattern (converging versus diverging flow at branch points), in permeability (De Clerck et al, 1985), in their thromboembolic reaction to microinjury (oude Egbrink et al, 1988), in the margination of leukocytes (Tangelder and Arfors, 1992) and in their platelet concentration distribution

Submitted for publication 
(Chapter 4; Woldhuis et al, 1992). It is of interest to note that, in contrast to arterioles, in venules the platelet concentration distribution differs from the one observed in vitro (Eckstein et all, 1988; Palmer, 1967; Tilles and Eckstein, 1987).

In the present study we have determined velocity profiles in venules, employing the same methodology as previously used for arterioles (Tangelder et al, 1986). To allow comparison of the profiles in both vessel types, we used the same tissue preparation, i.e. the rabbit mesentery, and the same range of diameters $(18-32 \mu \mathrm{m})$. As in arterioles, we have also tried to obtain least estimates of wall shear rate, and, using the plasma viscosity value of each animal, of wall shear stress.

\subsection{MATERIALS AND METHODS}

\section{Animals and intravital microscopy}

Experiments were performed on 5 New Zealand White rabbits of either sex, ranging in weight from 2.1 to $2.6 \mathrm{~kg}$ (mean $2.3 \mathrm{~kg}$ ). Anesthesia was induced by intramuscular injections of $40 \mathrm{mg} / \mathrm{kg}$ body weight ketamine hydrochloride (Nimatex, A.U.V., Cuijk, the Netherlands) and $4 \mathrm{mg} / \mathrm{kg}$ body weight xylazine hydrochloride (Sedamun, A.U.V.), and was maintained with a continuous intravenous infusion ( $15 \mathrm{ml} /$ hour) of the same substances (40 and $5 \mathrm{mg} / \mathrm{kg}$ bodyweight/hour, respectively), dissolved in a Lactetrol solution (Aesculaap, Boxtel, the Netherlands). This infusion was given through a polyethylene catheter (PE 60) in the femoral vein, and started about 60 minutes after induction of anesthesia. A polyethylene catheter (PE-60), positioned in the femoral artery and connected to an external pressure transducer (CTC, CP-01), was used to measure mean arterial blood pressure. Heart rate was derived from the instantaneous pressure pulses. The blood pressure catheter was continuously perfused with salline (5 $\mathrm{ml} /$ hour) via an Intraflo II-system (Sorenson Research); no heparin was used.

Blood was collected from a central ear artery in disodium ethylenediamine tetraacetic acid (EDTA, 0.1 vol, 0.027M). Platelets were counted with a Coulter Counter (model ZF) and hematocrit was measured with an Autocrit II centrifuge (Clay Adams). Plasma viscosity was assessed with a capillary viscometer at $37^{\circ} \mathrm{C}$ (Rheomed $\mathrm{GMbH}$, Aachen, Germany). According to Poiseuille 's law, flow velocity is inversely proportional to viscosity for a given driving pressure. In the capillary viscometer the plasma sample flows at a constant driving pressure through a polyethylene tube with 
inner diameter of $800 \mu \mathrm{m}$. The instrument was calibrated with two solutions with a known standardized viscosity (Rheomed GMbH, Aachen, Germany). All parameters were measured in duplicate, the means were taken and they were corrected for the dilution with EDTA.

After cannulating the trachea, the rabbits were ventilated with a mixture of $76 \%$ nitrogen, $22 \%$ oxygen and $2 \%$ carbon dioxide. The respiration rate was $60 /$ minute, and the tidal volume was about $18 \mathrm{ml}$. Blood gas analysis and hemoglobin measurements were performed using an acid-base analyzer (ABL 3 Radiometer) on arterial blood collected 10 minutes after onset of ventilation. Mean values ( \pm s.d.) were: $\mathrm{pH} 7.46 \pm$ $0.04, \mathrm{pCO}_{2} 31.2 \pm 2.6$ and $\mathrm{pO}_{2} 80.5 \pm 7.2 \mathrm{mmHg}$.

A short segment of the distal ileum was brought outside through a small abdominal midline incision. The mesentery was spread over a siliconized glass plate mounted on the stage of a Leitz intravital microscope adapted to telescopic imaging (Slaaf et al, 1982). During the experiments the mesentery was continuously superfused with a buffered Tyrode's solution ( $\mathrm{pH} 7.37 \pm 0.04 ; 37^{\circ} \mathrm{C}$ ). The bowels were kept moist with overlying wet gauze. Vessel diameters were measured off-line using a home-built video shearing device (Intaglietta and Tompkins, 1973).

Fluorescence microscopy was performed with an incident illuminator (Leitz Ploemopak 2.2, tubefactor 1.6x) with interchangeable filter set N 2.1, (excitation filter: BP 515-560, dichroic mirror: RKP 580, barrier filter: LP 580). A Leitz 100x salt water immersion objective (numerical aperture 1.2) was used. To give two flashes of sufficient intensity shortly following each other (see below), two Xenon flash arcs were used, each fed by its own power supply (Chadwick Helmuth). A mirror with $50 \%$ transmittance and $50 \%$ reflectance was used to bring the light from each lamp into the incident illumination pathway. Coupling between the electronic circuits of the two power supplies was achieved with optocouplers, to avoid unwanted discharge of the second unit at the moment of the first flash. Images were displayed on a TV monitor through an intensified CCD camera (Hamamatsu, type C2400-97) and recorded on videotape (Sony $U$-matic). Final magnification at the front plane of the TV camera was $200 x$.

In venules leukocytes often move in a rolling way along the endothelium at a velocity significantly lower than that of other blood cells. To evaluate a possible influence of this phenomenon on the velocity profile we counted off-line the number of leukocytes which showed this rolling behavior during the determination of the platelet velocity profiles. The fluorescent dye used to label the blood platelets (see below), also labels white blood cells without affecting the number of rolling leukocytes 
(Chapter 5; Woldhuis et al, in press). Because of the optics used for fluorescence microscopy, the depth of field for detection of rolling leukocytes was considerably smaller than the vessel diameter.

\section{Platelet velocity profiles}

A detailed description of the determination of platelet velocity profiles has been presented elsewhere (Tangelder et al, 1986). In short, circulating platelets were labelled by intravenous injection of the fluorescent dye acridine red in a dose of $6 \mathrm{mg} / \mathrm{kg}$ body weight (Tangelder et al, 1982a). The vessel wall is also labelled by the dye, but red blood cells are not. With position of sharp focus in the median plane of the vessel, i.e. the largest diameter, pairs of flashes were given with a short, preset time interval between both flashes (range 4-11 ms), yielding two images of the same platelet displaced over a certain distance. The time interval between two flash pairs (350-500 $\mathrm{msec}$ ) was sufficiently long to ensure that each flashed TV field contained no information from the previous flash pair. Depending on the moment that a flash pair was given (see below) with respect to the moment of read out of a TV-field by the CCD camera, the two images of a moving platelet were either displayed in the same TV-field, or one in the first TV-field and the second in the next one. Both situations yielded completely overlapping velocity values at all radial positions, and, hence, no distinction was made between these two situations.

All flash pairs were given at the same moment in the cardiac cycle, using as trigger the peak in femoral artery pressure. As illustrated in Figure $6.1 \mathrm{~A}$, in all experiments this moment coincided for the majority of the flash pairs with the end of the diastolic phase of flow velocity in the venule. An instantaneous red blood cell velocity recording was obtained prior to each period of assessment of the velocity profile, using a prism grating system (Slaaf et al, 1981) coupled to a correlator (IPM, San Diego). Images were recorded at vessel sites without upstream branch points within at least 10 vessel diameters. Curvatures of the vessels immediately up or downstream of the site of measurement were avoided.

Video recordings were analyzed frame by frame. A transparency was placed over the monitor screen. Both vessel walls and the centroids, i.e. the centers of gravity, of the two images of a blood platelet were identified by eye and marked on the transparency. These centroids were used to measure (see Figure 6.1B): 1) the displacement of the blood platelet in the preset time interval, yielding its velocity, and 2) its distance (d) from the left vessel wall, defined according to the direction of flow. This distance $d$ and the 

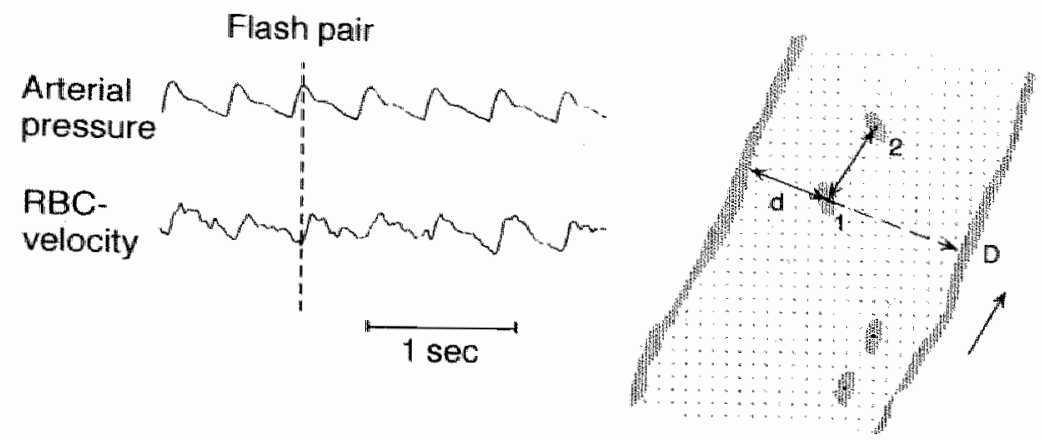

Figure 6.1: Position of flash pairs in cardics cycle (A), and parameters measured to determine the platelet velocity profile $(B)$. Flash pairs were triggered using the femoral artery pressure. Its peak mostly coincided with the diastolic flow phase in a venule, as determined from red blood cell (RBC) velocity. In $B$, two images of the same platelet are depicted schematically ( 1 and 2). Their centers of gravity (dot) are displaced over a certain distance during the time interval (4-11ms) between the 2 flashes of a pair. This yields the velocity of the platelet. Its relative radial position was determined from its distance to the left vessel wall (d) and vessel diameter $(D)$.

vessel diameter (D) at that specific point were used to calculate the relative radial position ( $r / R$ ) of the platelet at that moment according to: $r / R=$ [(d/D) -0.5$] \times 2$. For the two images of a platelet, the mean of the two relative radial positions was taken. Velocities were displayed as a function of this mean relative radial position, rendering the velocity profile. The number of platelets counted in the various vessels for the assessment of a velocity profile ranged from 86 to 188 with a mean of 120 (standard deviation \pm 30 ).

We used only pairs of images that were reasonably sharp in focus and clearly belonged to the same platelet. This means that, with the high numerical aperture of the objective lens and the total optical magnification used, the data were obtained from an optical section about the median plane of the vessel with a depth of about $5 \mu \mathrm{m}$ or less (Tangelder et al, $1982 \mathrm{~b}$ ). Because the centroid of a blood platelet image is used for the measurements, in principle no data points can be obtained closer to the wall than about $0.5 \mu \mathrm{m}$, because of the size of blood platelets (Teirlinck et al, 1984). 


\section{Chapter 6}

\section{Velocity profile shape: curve fitting}

The obtained velocity profiles were described with the following equation, as previously used in arterioles (Tangelder et al, 1986):

$$
\begin{array}{r}
V(x)=V_{\max }\left(1-|a(I / R)+b|^{k}\right), \\
-1 \leq r / R \leq+1, \quad a>0
\end{array}
$$

where $V(r)$ is the velocity at radial position $r, V_{\text {MAX }}$ is the velocity in the vessel center, $R$ is the radius of the vessel, a is a scale factor which allows to fit the data with a curve which is not forced through zero velocity at the wall (no-slip condition; see next paragraph), $\mathbf{k}$ is a factor which describes the degree of flattening of the profile in this remaining core, while $b$ is $a$ parameter correcting for a shift of the top of the profile away from the vessel center. The vertical stripes denote absolute values. In case factor a is $1, k$ is 2 and $b$ is zero, a parabolic velocity distribution is obtained. Fitting the data with equation 1 , or with three restricted versions of it in which we fixed several factors (see results), was performed with the use of the statistical package BMDP (modules $1 R$ and 3R; UCLA, Ca). In all cases tolerance of the different parameters exceeded 0.04 , indicating negligible multicollinearity between the fitted variables.

It should be pointed out that the aim of the fits was to draw a proper line through all experimental points, and not to extrapolate beyond the range of measurements. Therefore, a fit yielding a value for parameter a smaller than 1, and, hence, an extrapolated positive intercept of the fit with the vessel wall, should not be interpreted as prediction of a non-zero velocity at the vessel wall. The latter is not realistic because flow velocity at the wall is likely to be zero due to the no-slip condition (Caro et al, 1978). Because no data points could be obtained close to the wall, no line was fitted between vessel wall and transition point, defined as the mean of the relative radial positions of the three platelets closest to a vessel wall. If parameter a is significantly smaller than 1 , a layer of fluid at the wall must exist with an apparent viscosity which is lower than that in the remainder of the vessel. If factor $k$ is significantly larger or smaller than 2 , the shape of the profile in this remaining core will be flattened or more pointed, respectively, as compared to a parabola.

The ratio of $V_{\text {MAX }}$ and the mean velocity of the fitted profile $\left(V_{\text {MENN }}\right)$ describes to which extent the fitted profille varies between plug flow or a parabola. This ratio is 1 in the case of complete plug flow, i.e. all layers of fluid travelling at the same velocity, and 2 in the case of a parabolic profile. However, this ratio does not give any information about the actual shape of 
the profile, since several pairs of parameters a and $\mathrm{k}$ may yield the same value of this ratio. As previously described (Tangelder et al, 1986), the ratio of $\mathrm{V}_{\text {MAX }}$ and $\mathrm{V}_{\text {MEAN }}$ can be approximated by:

$$
\frac{V_{\max }}{V_{\operatorname{maan}}}=(K+2) /\left(K+2-2(a)^{k}\right)
$$

if factor $\mathrm{b}$ is neglected. Since parameter $\mathrm{b}$ was indeed small in all cases (see results), this equation was used.

To evaluate whether the velocity in the venules remained stationary during assessment of the profiles (43-236 seconds), a serial number was assigned to each measurement. The velocities of platelets flowing at a relative radial position of less than 0.3 were plotted against their serial number, and fitted by linear regression. The slopes of these lines were used to calculate the percentage change in velocity during the measuring period. As compared to the velocity at the start of a measuring period, which was designated $100 \%$, platelet velocity at the end did not differ significantly (range from 97 to $123 \%$, median: $110 \%$ ).

\section{Least estimates of shear values}

The fitted curve was assumed to describe the actual velocity profile from the vessel center onto and including the transition point at each wall (TrP; see above). From the transition point to the vessel wall, velocity has to drop to zero to satisfy the no-slip condition (Caro et al, 1978). To obtain a least estimate of wall shear rate (WSR), we assumed a linear velocity profile in this fluid layer. Compared to the assumption of a parabolic profile in this layer, values obtained by assuming a linear profille are only less than $4 \%$ smaller. WSR was calculated according to:

$$
W S R=\frac{2 \cdot V_{\| T R P}}{D \cdot(1-|x|)}
$$

where $V_{(\mathrm{rr})}$ is the velocity at the transition point, $\mathrm{D}$ is the average vessel diameter and $\mathrm{x}$ is the relative radial position of the transition point. An average value for the vessel diameter had to be used because along the length of a vessel segment analyzed $(30-40 \mu \mathrm{m})$ the diameter often varied. 


\section{Chapter 6}

From the least estimate of wall shear rate (WSR; in reciproca seconds) and plasma viscosity (in $\mathrm{mPa}$ s), which was measured in each animal, a least estimate of wall shear stress (WSS; $\mathrm{mPa}$ ) can be obtained with the use of the following equation:

$$
\text { WSS }=\text { WSR } . \text { Plasma viscosity }
$$

We used plasma viscosity, because the apparent viscosity of the layer of fluidat the wall will not be lower than plasma viscosity, it might even be higher, and it was our aim to obtain a least estimate of wall shear stress. The extent to which wall shear rate (WSR) values, as derived from the actual velocity profiles, are higher than those calculated assuming a parabolic velocity distribution for the same volume flow (WSR-P), was estimated with the following equation (Tangelder et al, 1988):

$$
\frac{W S R}{W S R-P}=\frac{0.25 \cdot V_{(T I P)}}{(1-|x|) \cdot V_{\text {MEAN }}}
$$

This ratio can also be used as a least estimate of the apparent viscosity of blood in the microvessel relative to the viscosity of plasma (Tangelder et al, 1988; Reneman et al, in press). As mentioned before, the estimate of wall shear stress approximates wall shear rate (WSR) times plasma viscosity. Let us consider a microvessel through which at the same volume flow blood is flowing as a Newtonian fluid. In that case the velocity profile parabolic and wall shear stress will equal WSR-P times apparent blood viscosity. For both situations the pressure drop in the vessel is proportional to both wall shear stress and apparent viscosity (Caro et al, 1978; Lipowsky et al, 1978). Therefore, the ratio of the apparent viscosity of blood and the viscosity of plasma, being the relative apparent viscosity of blood is equal to the ratio of WSR/WSR-P. Multiplication of this ratio with the plasma viscosity then yields the apparent blood viscosity in the microvessel.

\section{Statistical analysis}

Data were analyzed with SPSS/PC+ statistical package (SPSS, Chicago, IL). In all tests the level of significance was set at $5 \%$. Correlations were performed with Spearmans nonparametric rank correlation test. Nonpaired data groups were compared using the Mann-Whitney U test. To test 
the median of one data group against a theoretical value, we used as nonparametric alternative to the one-sample t-test, the paired Wilcoxon-test (Bradley, 1968). This procedure requires sufficient symmetry of the data in the group, which was tested with the Kolmogorov-Smirnov goodness of fit test for normality; for all velocity profile parameters (equations 1 and 2), as well as WSR/WSR-P, p-values were between $0.58-1.00$.

\subsection{RESULTS}

The systemic hematocrits of the animals ranged from 38 to $41 \%$ (mean: 39\%), systemic platelet counts from 362 to $477 \times 10^{\circ} / 1$ (mean: $405 \times 10^{9} / 1$ ) and hemoglobin from 8.3 to $9.5 \mathrm{mmol} / 1$ (mean: $8.8 \mathrm{mmol} / \mathrm{l}$ ), which are all values within the normal ranges for rabbits (Kozma et al, 1974). All animals started with a mean arterial blood pressure above 58 $\mathrm{mmHg}$; blood pressures during assessment of the velocity profiles are presented in table 6.1. Concomitant heart rates ranged from 118-128 beats per minute $(\mathrm{b} / \mathrm{m})$, except for one stray value of $140 \mathrm{~b} / \mathrm{m}$ (median $124 \mathrm{~b} / \mathrm{m}$, including all data). Plasma viscosity ranged from 0.97 to $1.02 \mathrm{mPa} . \mathrm{s}$ (median 0.99 mPa.s; see table 6.2).

\section{Velocity profiles}

Velocity profiles were obtained in eight venules (see table 1 ) in five animals. Figures 6.2A, 6.3A and 6.4A show examples of these profiles. Each dot represents the velocity of one platelet. All profiles were rather symmetric. Because of this, we neglected in the fits with the three restricted versions of equation 1 (see below) parameter $b(b=0)$.

Figure $6.2 \mathrm{~A}$ also presents the best fits with equation 1 and with a parabola, i.e. $a=1$ and $k=2$ in equation 1 (first restricted version). Fitting with a parabola did not yield a proper result. This is clear from Figure 6.2A, as well as from Figure 6.2B, which shows the residuals of this fit. As can be seen from the pattern of residuals, the parabola underestimates the platelet velocities near the wall and overestimates the velocities in the center of the vessel. The same pattern was observed in 5 other venules. In the 2 remaining venules the parabola tended to underestimate the velocities in the center, while near the wall no systematic over- or underestimation was apparent.

Figure 6.3A shows another profile as well as the best fits with equation 1 and with a second restricted version of it in which $a=1$ and 

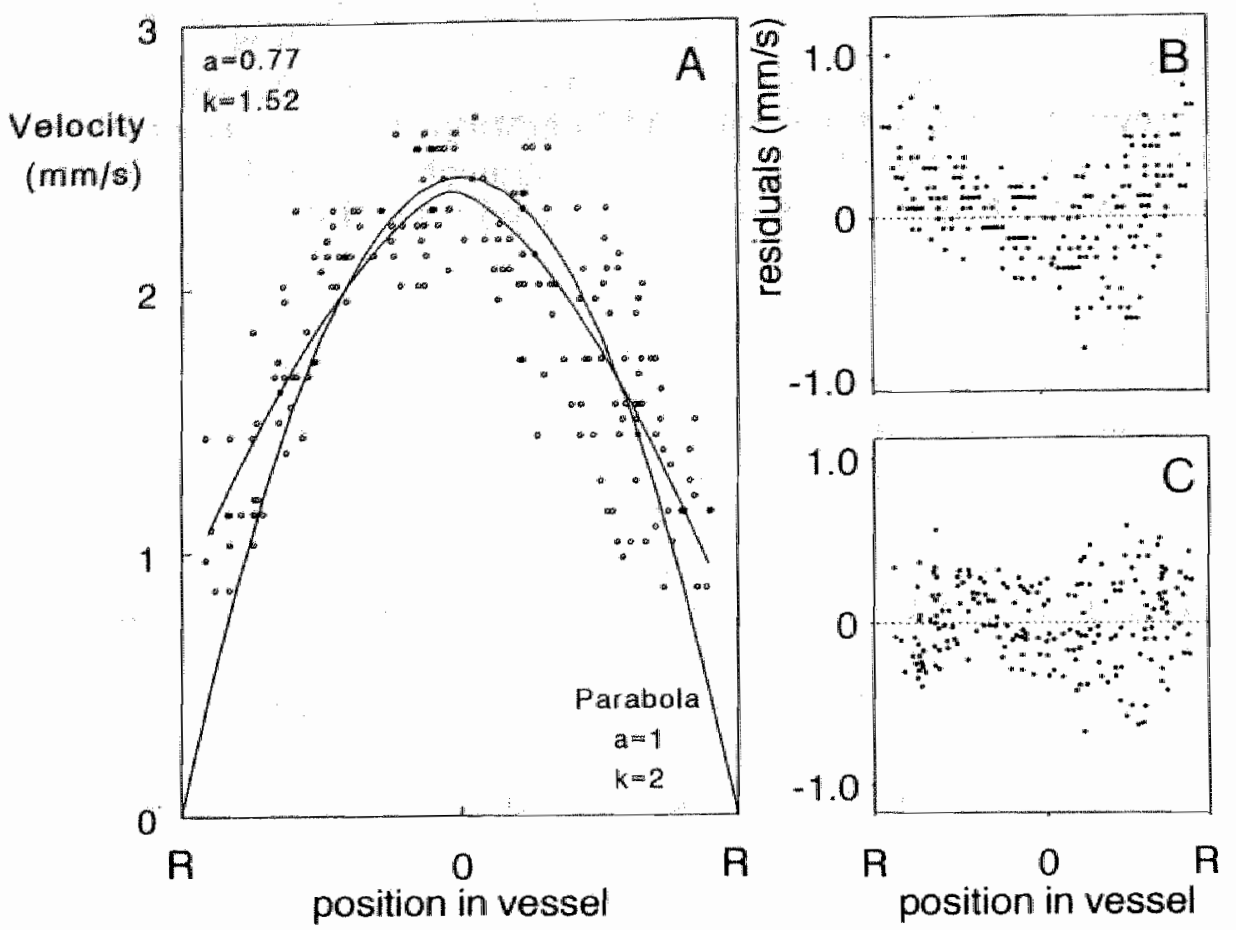

Figure 6.2: Velocity profile of blood platelets flowing in a 25 un venule (A). In addition, the best fits as obtained with a parabola ( $a=1, k=2, b=0$ in equation 1$)$, which describes a onefluid model, and with equation 1 ( $a, k$ and $b$ all free) are shown. The residuals of the fit with the parabola are shown in $B$ and those of the fit with equation 1 in $C(b=0.025)$. At the dashed line in $B$ and $C$, a data point coincides with the fitted curve. Number of platelets $=188$. Flash intenval: $4 \mathrm{~ms}$.

$k=$ free. This version still implies a one-fluid model, i.e. a theory in which the flow properties of blood are assumed to be uniform over the whole cross-sectional area of the vessel. However, values of $\mathrm{k}$ smaller than or exceeding a value of two indicate a more pointed or flattened profile as compared to a parabola, respectively. This one-fluid model with $k=$ free, still did not yield a good description of the profiles, as can be seen in Figures 6.3A and 6.3B. In most experiments the fits tended to underestimate the velocities near the wall as well as in the vessell center, and to overestimate them in between. Values of $k$ ranged from 2.51 to 3.06 , except for two values of 1.72 and 1.74 (median: 2.75 ). These values would indicate a 

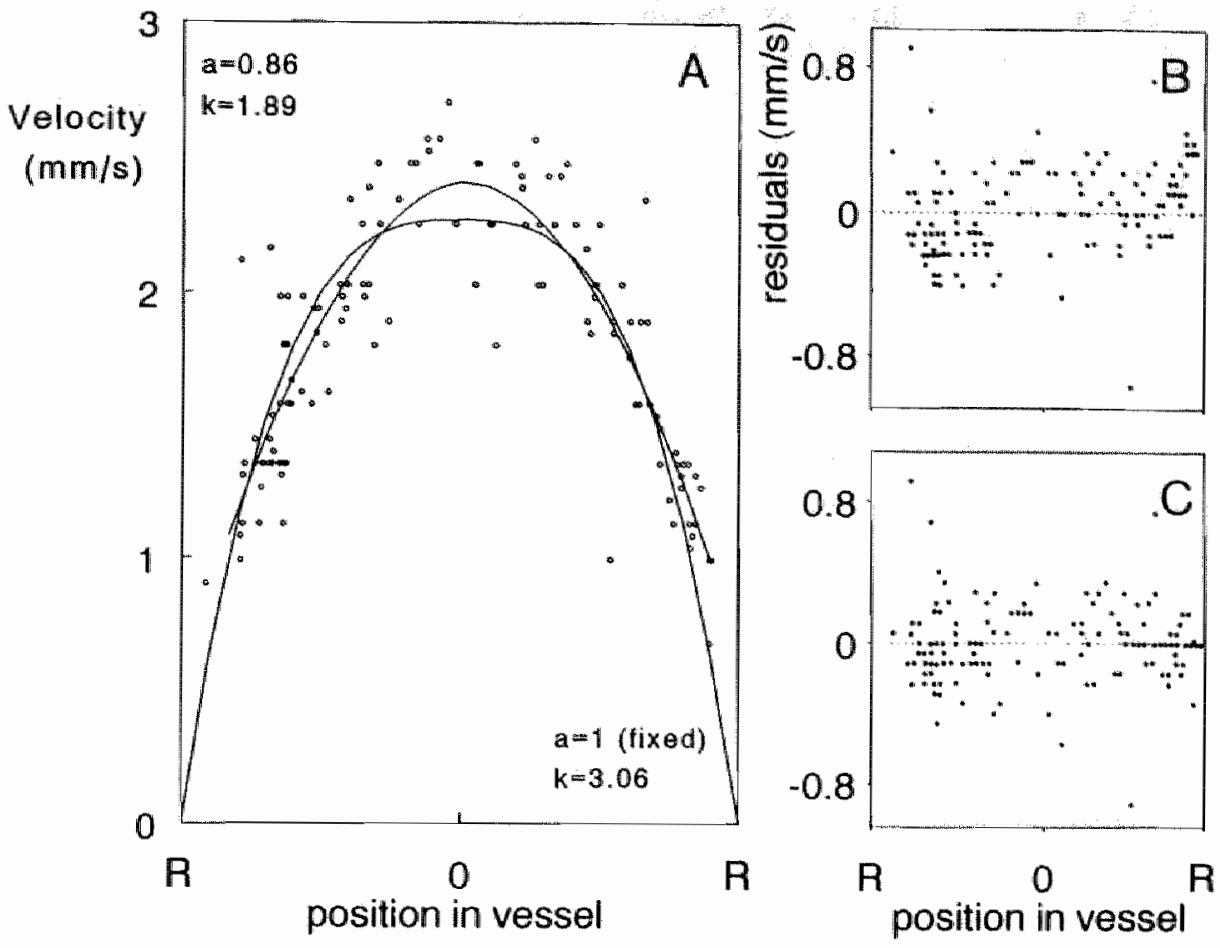

Figure 6.3: Velocity profile of blood platelets flowing in a $32 \mu \mathrm{m}$ venule (A). In addition, the best fits as obtained with a second restricted version of equation $1(a=1, k=f r e e, b=0)$, i.e. $a$ one-fluid model with blunted profile, and with equation $I$ ( $a, k$ and $b$ all free) are shown. Residuals are presented in $B$ and $C(b=-0.018)$, respectively. Number of platelets $=127$. Flash interval: $5 \mathrm{~ms}$.

considerable blunting in the vessel center in the majority of the experiments, which was not in agreement with visual inspection of the data.

By contrast, a rather good description of the profiles was obtained with a two-fluid model. Figure $6.4 \mathrm{~A}$ presents a third profile as well as the best fits with equation 1 and with a third restricted version of it in which $a=$ free and $k=2$. With this latter version, the residuals scattered remarkably well around zero at all radial positions, as is shown in Figure 6.4B. This was found in all experiments. Values of factor a as found with this third restricted version of equation 1 ranged from 0.86 to 1.02 (median: 0.90 ). This supports the idea of a need for a two fluid model to describe the velocity profiles. 


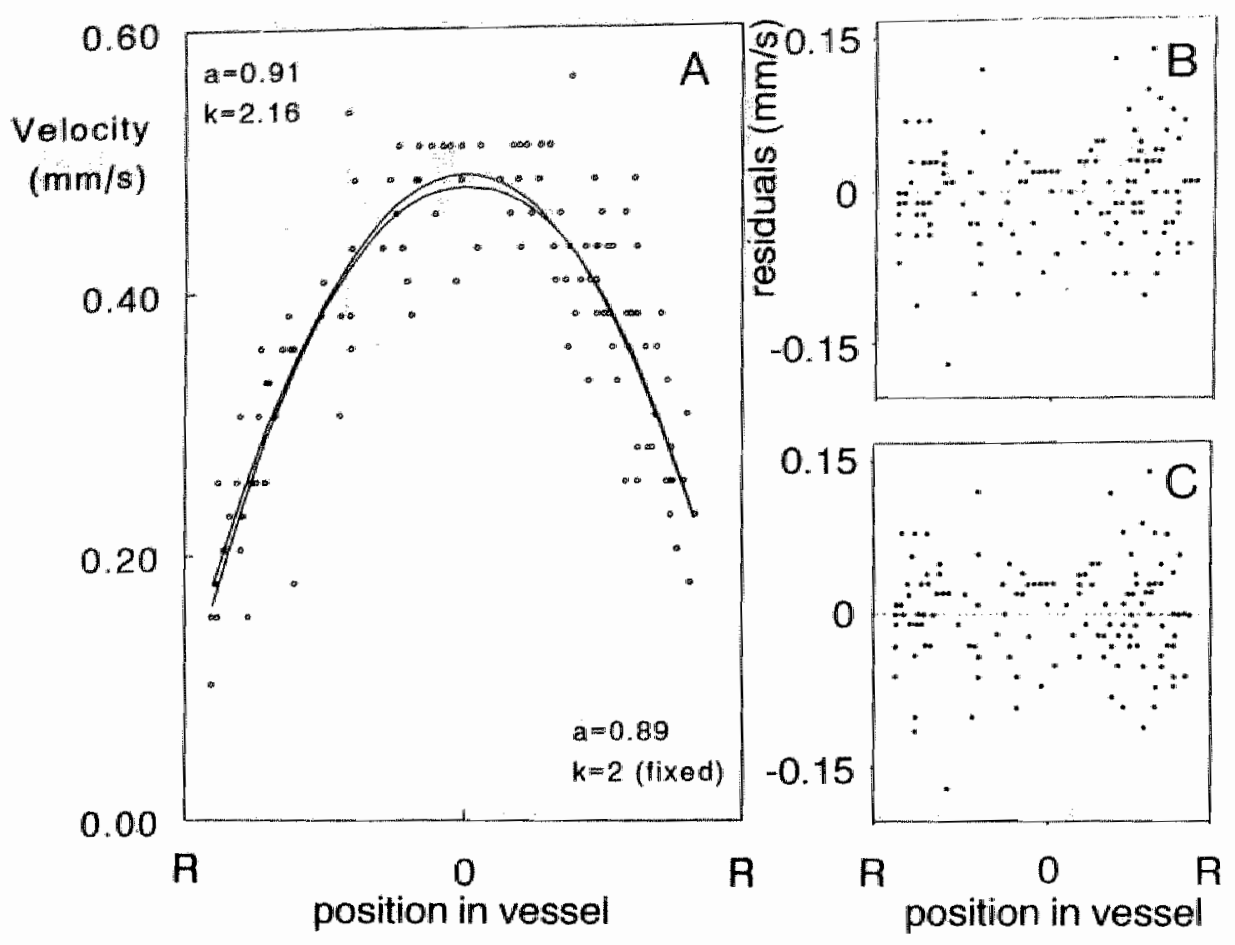

Figure 6.4: Velocity profile of blood platelets flowing in a $22 \mu \mathrm{m}$ venule (A). In addition, the best fits as obtained with a third restricted version of equation 1 ( $a=f r e e, k=2, b=0)$, i.e. $a$ two fluid-model with a parabolic profile in the core, and with equation 1 ( $a, k$ and $b$ all free) are shown. Residuals are presented in $B$ and $C(b=-0.001)$, respectively. Number of platelets $=126$. Flash interval: $9 \mathrm{~ms}$.

Examples of fits with equation 1, i.e. without restricted parameters, are also presented in Figures $6.2 \mathrm{~A}, 6.3 \mathrm{~A}$ and $6.4 \mathrm{~A}$, with the concomitant residuals in $6.2 \mathrm{C}, 6.3 \mathrm{C}$ and $6.4 \mathrm{C}$, respectively. In all venules, the residuals scattered around zero at each relative radial position, with no systematic under- or overestimation of velocities. Values of $b$ scattered around zero, indicating no systematic shift of the profiles to one vessel wall. Absolute values of $b$ as obtained in the different experiments ranged from 0.0009 to 0.0252 (median 0.0092), confirming that in the different experiments the top of the profile was indeed positioned close to the vessel center.

The values of the other profile parameters as obtained with equation 1 (all parameters free), and of the ratio $V_{\text {MAX }} / V_{\text {MEAN }}$ as calculated with 
equation 2, are presented in table 6.1. During assessment of the profile in 2 vessels, mean arterial pressure was relatively low (see table 6.1). However, the velocity profile parameters in these 2 vessels scattered within the distributions of the other vessells. Moreover, no correlation was found between mean arterial pressure or heart rate on the one hand and any of these parameters on the other, suggesting no significant influence of these systemic hemodynamic parameters on the shape of the velocity profile in venules.

In all venules the fit with equation 1 (all parameters free) yielded velocities near the vessel wall which were higher than expected in the case of a parabolic profile, as is indicated by parameter a being significantly smaller $(p<0.02)$ than unity (range $0.77-0.99$; median 0.90 ; table 6.1). This finding indicates that blood flow in small venules cannot be described with a one-fluid model, and points to the existence of a thin layer of fluid at the wall with a significantly lower apparent viscosity than the fluid in the remaining core. The same was already suggested by the fits with the above mentioned third restricted version of equation $1(a=$ free and $k=2)$. The good fits obtained with this third version of equation 1, also suggested that

Table 6.1: Platelet velocity profile parameters as determined in venulles in vivo.

\begin{tabular}{|c|c|c|c|c|c|}
\hline \multirow{2}{*}{$\begin{array}{l}\text { Venular } \\
\text { diameter } \\
(\mu \mathrm{m})\end{array}$} & \multirow{2}{*}{$\begin{array}{l}\text { Mean } \\
\text { arterial } \\
\text { pressure* } \\
(\mathrm{mmHg})\end{array}$} & \multicolumn{4}{|c|}{ Velocity Profile Parameters\# } \\
\hline & & $\begin{array}{l}\mathrm{V}_{\mathrm{MAX}} \\
(\mathrm{mm} / \mathrm{s})\end{array}$ & a & k & $\mathrm{V}_{\text {MAX }} \mathrm{N}_{\text {MEN }}$ \\
\hline 18 & 60 & 0.49 & 0.89 & 1.4 & 2.03 \\
\hline 21 & 83 & 1.23 & 0.93 & 2.3 & 1.64 \\
\hline 22 & 79 & 0.48 & 0.91 & 2.2 & 1.64 \\
\hline 24 & 50 & 1.12 & 0.99 & 2.6 & 1.76 \\
\hline 25 & 78 & 2.37 & 0.77 & 1.5 & 1.62 \\
\hline 26 & 82 & 1.85 & 0.95 & 1.5 & 2.13 \\
\hline 28 & 38 & 0.29 & 0.86 & 1.7 & 1.73 \\
\hline 32 & 68 & 2.41 & 0.86 & 1.9 & 1.63 \\
\hline median & 73 & 1.18 & 0.90 & 1.8 & 1.69 \\
\hline
\end{tabular}

- As present during the measurement period.

\# See for $V_{\text {max }}$ i.e. the maximal velocity of the fitted curve, $k$ and $a$, Equation 1; for $\mathrm{V}_{\max } / \mathrm{V}_{\text {mean }}$, i.e. the ratio of $\mathrm{V}_{\max }$ and the mean velocity of the theoretical profile, Equation 2. 
Table 6.2: Least estimates of wall shear values calculated from velocity profiles as measured in venules in wivo.

\begin{tabular}{|c|c|c|c|c|c|}
\hline \multirow{2}{*}{$\begin{array}{l}\text { Venular } \\
\text { diameter } \\
(\mu \mathrm{m})\end{array}$} & \multirow{2}{*}{$\begin{array}{l}\text { Plasma } \\
\text { viscosity } \\
\text { (mPas) }\end{array}$} & \multirow{2}{*}{$\begin{array}{l}\text { Distance between } \\
\text { transition point\# } \\
\text { and vessel wall } \\
(\mu \mathrm{m})\end{array}$} & \multicolumn{3}{|c|}{ Least estumates of \# } \\
\hline & & & $\begin{array}{l}\text { WSR } \\
\left(\mathrm{s}^{-1}\right)\end{array}$ & WSR/WSR-P & $\begin{array}{l}\text { WSS } \\
(\mathrm{mPa})\end{array}$ \\
\hline 18 & 1.03 & 1.9 & 97 & 0.92 & 100 \\
\hline 21 & 1.02 & 1.8 & 306 & 1.07 & 312 \\
\hline 22 & 0.98 & 1.5 & 130 & 1.22 & 127 \\
\hline 24 & 0.99 & 2.0 & 218 & 1.04 & 216 \\
\hline 25 & 1.02 & 1.3 & 814 & 1.73 & 830 \\
\hline 26 & 0.98 & 1.9 & 257 & 0.96 & 252 \\
\hline 28 & 0.97 & 2.0 & 59 & 1.19 & 57 \\
\hline 32 & 0.99 & 2.2 & 501 & 1.28 & 496 \\
\hline median & 0.99 & 1.9 & 238 & 1.13 & 234 \\
\hline
\end{tabular}

\# Transition point = mean of relative radial position of the 3 platelets closest to a vessel wall, WSR = wall shear rate (see equation 3 ), WSR-P = WSR of a parabolic velocity distribution but with the same volume flow in the vessel (see equation 5), WSS = wall shear stress (see equation 4), \# and \#\# For each experiment the mean of values obtained at right and left vessel wall is presented.

the fitted value of parameter $\mathrm{k}$ would be close to 2 . Indeed, as is shown in table 6.1, $\mathrm{k}$ did not differ significantly from 2 (range 1.4 to 2.6 ; median 1.8 ). On the other hand, parameter $\mathrm{V}_{\text {MAX }} / \mathrm{V}_{\text {MEAN }}$ was significantly smaller than 2 (range: 1.63-2.13; median $1.69 ; \mathrm{p}<0.04$ ), indicating that in venules the velocity profiles differ from a parabola, but are generally closer to it than to plug flow. No correlations were found between diameter or $\mathrm{V}_{\mathrm{MAx}}$ on the one hand and parameters $a, k$ or $V_{\text {MAX }} / V_{\text {MEAN }}$ on the other. The same holds for the number of rolling leukocytes, which ranged from 3 to 20 per minute (median: 10 leukocytes /minute), as correlated with the latter three parameters.

\section{Least estimates of shear values}

The least estimates of the average wall shear rates (WSR) as calculated for each venule are presented in table 6.2 (range: 59-814 s' median $\left.238 \mathrm{~s}^{-1}\right)$. Vessel diameters are presented as well, to allow comparison with table 6.1, which shows the centerline velocities $\left(V_{\text {MAX }}\right)$ in these venules 
$(0.3-2.4 \mathrm{~mm} / \mathrm{s} ;$ median $1.2 \mathrm{~mm} / \mathrm{s})$. These shear rates were 0.92 to 1.73 (median 1.13) times higher than expected in the case of a parabolic profile with the same volume flow (WSR/WSR-P in table 6.2), being a nonsignificant difference. Least estimates of wall shear stress (WSS) ranged from 57 to $830 \mathrm{mPa}$ (median: $236 \mathrm{mPa}$; table 6.2), and of apparent blood viscosity from 0.93 to $1.77 \mathrm{mPa}$.s (median: $1.13 \mathrm{mPa} . \mathrm{s}$ ). The least estimates of wall shear rate at opposite walls in a vessel did not differ much. In the various experiments, the values found at the left and right vessel wall differed from the mean value for that vessel (presented in table 6.2) by 0 21\% (median: $7.0 \%$ ).

Because the transition points used for the calculations were located relatively far away from the vessel wall (range: 1.3-2.2 $\mu \mathrm{m}$; median: $1.9 \mu \mathrm{m}$; see table 6.2), we also used the outermost data-point near each vessel wall as transition point. Vessel means of the outermost data-points ranged from 1.1-1.9 $\mu \mathrm{m}$ (median: $1.6 \mu \mathrm{m}$ ). The least estimates obtained in this way differed only slightly from those presented in table 6.2 , with WSR values ranging from 66 to $850 \mathrm{~s}^{-1}$ (median: $246 \mathrm{~s}^{-1}$ )and WSR/WSR-P values ranging from 1.00-1.82 (median: 1.20 ).

\subsection{DISCUSSION}

The present study shows that the velocity profile in small venules cannot be described adequately with a Newtonian one fluid model, i.e. a parabolic velocity profile. Proper description requires a two fluid model: a thin layer of fluid at the wall with a significantly lower apparent viscosity than the fluid in the remaining core of the vessel. In this core, the shape of the profile did not differ significantly from that of a parabola. The velocities near the venular wall, however, were higher than expected in case of a parabolic profile. This is indicated by the fact that factor a (table 6.1) was significantly smaller than unity. Therefore, the velocity profile in venules is more blunt than a parabolic profile. This has also been suggested by studies in other tissues (Schmid-Schonbein and Zweifach, 1975; Rosenblum, 1976). In contrast to our study, however, these older studies lacked the resolution to provide a detailed profile in the core of the vessel. In this core we found that the shape of the velocity profile could be fitted adequately with a parabola if it was not forced through zero velocity at the wall. Although this yielded a satisfactory description of the shape of the profiles in the vessel center, it cannot be excluded that in several venules the profile in the vessel center is more pointed than expected on the basis of a parabola, as 
indicated by the lowest values for $\mathrm{k}$ in table 6.1. Nevertheless, fitting with the two fluid version of equation 1, i.e factor a left free and factor $k=2$, yielded a good fit in all cases. This also indicates that after a steep rise in velocity near the venular wall, the profile in the remaining core can be described by a parabola.

The observed velocity profiles were rather symmetric with the top of the profile positioned close to the vessel center. This finding indicates that disturbances of the profile due to side branches had mostly disappeared after approximately 10 vessel diameters. Upstream branch points were at least this distance away from the site of observation, while curves immediately up-or downstream were avoided. Another possible factor might have been that in this tissue differences in local hematocrit between the confluencing venules were limited. It is a common observation that in venules flow is stratified, i.e. contributions from different branches tend to stay separate on their respective streamlines and show little tendency to mix. In this respect it is also interesting to note that the shape of the profiles in the central fluid-core did not differ significantly from a parabola. This suggests that these profiles had reached their final shape, indicating a limited entrance length. In case of a Newtonian fluid, the length needed for full development of the profile in a microvessel is about one vessel diameter, because viscous forces largely dominate over inertial forces (Caro et al, 1978). However, the actual entrance length for blood flowing through a microvessel is unknown.

The method used in this study, which employed fluorescent platelets as small and natural markers of flow, has been applied previously in arterioles. They had a similar diameter (range 17-32 $\mu \mathrm{m}$; median $24 \mu \mathrm{m}$ ) and were also located in the rabbit mesentery. Figure 6.5 compares the velocity profile parameters as found in the venules of this study with those obtained in these arterioles. For this comparison only measurements were included performed during the diastolic flow phase in the vessel. Parameter a did not differ between both vessel types, and was significantly smaller than unity in both groups. Hence, adequate description of the velocity profile requires a two fluid model in both arterioles and venules. By contrast, the shape of the profile in the core-fluid was significantly more blunt in arterioles than in venules, as is indicated by the higher values of parameter $k$ in the first type of microvessel. Due to this latter difference, mean velocity is closer to centerline velocity in arterioles than in venules. This is shown by the significantly lower values of $V_{\text {MAX }} / V_{\text {MEAN }}$ in arterioles than in venules. Hence, estimation of mean velocity and flow from photometric assessments of centerline velocity, which yield a weighted average of the velocity 


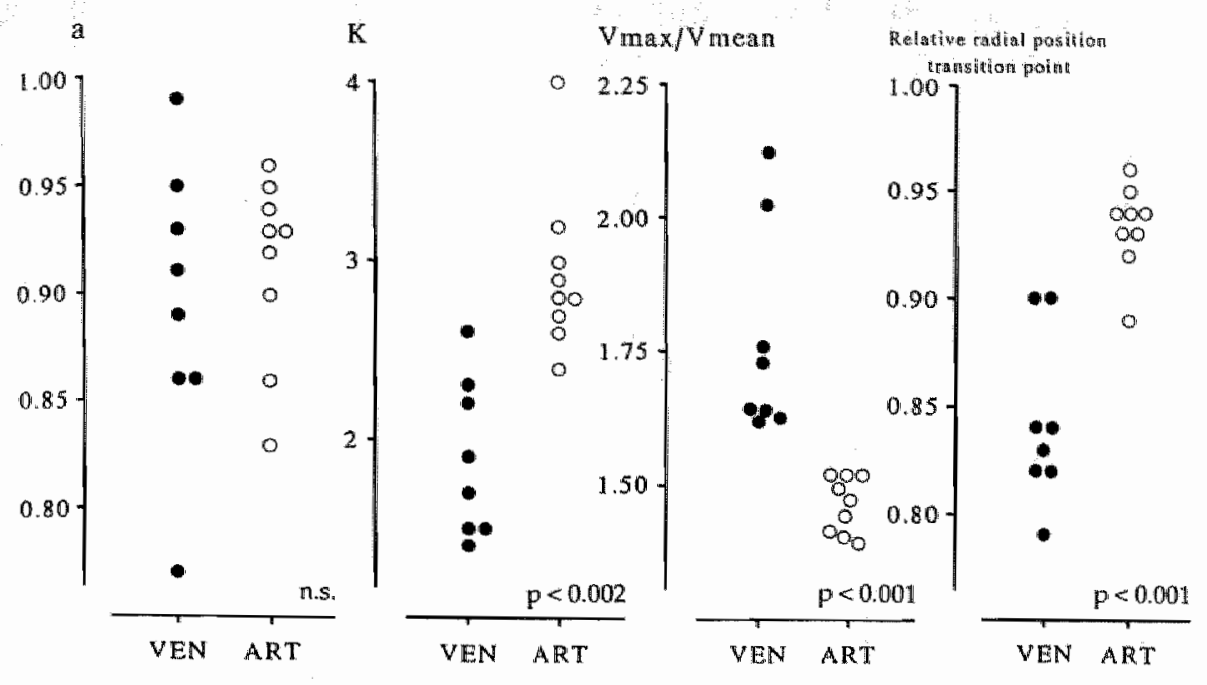

Figure 6.5: Comparison of velocity profile parameters in venules (ven; filled circles) and arterioles (ant; open circles). Vessel diameters did not differ, ranges being 18-32 um (median: $24.5 \mu \mathrm{m}$ ) and $17-32 \mu \mathrm{m}$ (median: $24 \mu \mathrm{m}$ ), respectively. Centerline velocities ( $V_{\max }$ ) ranged $0.3-2.4 \mathrm{~mm} / \mathrm{s}$ (median: $1.2 \mathrm{~mm} / \mathrm{s}$ ) and $1.3-14.4 \mathrm{~mm} / \mathrm{s}($ median: $3.1 \mathrm{~mm} / \mathrm{s}$ ), respectively. Measurements were performed during the diastolic flow phase in both vessel types. For $a, k$ and $V_{\max } V_{\text {mean }}$ see table 6.1, and for transition point see table 6.2.

distribution, would need a different correction factor in venules than in arterioles (Slaaf et al, 1984).

A second difference between arterioles and venules is the position of the transition point, defined as the mean of the relative radial positions of the three platelets closest to a vessel wall. This point is significantly further away from the wall in venules (median $1.9 \mu \mathrm{m}$; see table 6.2) than in arterioles (median $0.8 \mu \mathrm{m}$; Tangelder et al, 1986). Because the geometric center of a platelet image was used, and considering the size of the platelets, it was in principle impossible to find data points closer to the vessel wall than about $0.5 \mu \mathrm{m}$. Hence, the difference in transition point indicates the existence of an almost platelet free layer near the venular wall, in contrast to arterioles in which many flowing platelets can be observed in apparent contact with the vessel wall (Tangelder et al, 1986). A relatively large wall exclusion of platelets in venules was also observed in a study on the platelet concentration distribution, in which this exclusion appeared not to be caused by the presence of rolling leukocytes in these vessels (Chapter 
4; Woldhuis et al, 1992). Hence, the wall exclusion of platelets in venules, but not in arterioles, suggests a difference in surface properties between venular and arteriolar endothelium.

Regarding such a difference in surface properties between venular and arteriolar endothelium, it is surprising that the major difference in velocity profile between these vessel types was found in the vessel center. The diameters in both groups did not differ. It is also unlikely that this difference is caused by differences in cross-sectional shape of the vessels. In a previous study we found little difference in eccentricity, i.e. ratio of short to long axis of the vessel cross-section, between venules and arterioles (Chapter 4; Woldhuis et al, 1992). In fixed mesentery (Fox et al, 1980), as well as in other tissues (Kovacs et al, 1975), almost circular venules and arterioles can be found. Moreover, in both venules (this study) and arterioles (Tangelder et al, 1986) rather symmetric profiles were found with their top positioned close to the vessel center.

Another cause for the finding of central blunting in arterioles, but not in venules, might be differences in flow velocity. In the arterioles presented in Figure 6.5 , centerline velocities ranged from 1.3 to $14.4 \mathrm{~mm} / \mathrm{s}$ (median $3.1 \mathrm{~mm} / \mathrm{s})$, which is higher $(\mathrm{p}<0.01)$ than those observed in the venules of this study (see table 6.1). However, if the 3 arterioles with the lowest and the 3 venules with the highest centerline velocities are compared (see table 6.3), still a significant difference in factor $\mathrm{k}$ was found. Moreover, in the study on arterioles no difference was found between the degree of blunting and centerline velocity (Tangelder et al, 1986). Therefore, it is unlikely that the presence of central blunting of the velocity profile in arterioles, but not in venules, is due to differences in centerline velocity. It is also unlikely that this difference in central blunting is due to pulsatile flow, since the latter is present not only in arterioles, but is also commonly found in mesenteric venules (this study; Slaaf et al, 1981; oude Egbrink et al, 1991a). However, both vessel types differ in flow at branch points. The present study leaves open the possibility that the confluencing flow in venules contributes differently to the shape of the velocity profile than the diverging flow at arteriolar branch points.

The observed difference in velocity profile between venules and arterioles might also be related to differences in concentration distribution and flow behavior of the different blood cells. Platelet distribution, for example, differs greatly between both vessel types. In the vessel center, platelet concentration is approximately twice as high in venules as compared to arterioles. However, it is unlikely that differences in platelet distribution per se are responsible for the observed central blunting in arterioles, but not 
Table 6.3: Comparison of the 3 venules $(V)$ with the highest and the 3 arterioles $(A)$ with the lowest centerline velocity.

\begin{tabular}{llllc}
\hline & diameter $(\mu \mathrm{m})$ & $\mathrm{V}_{\max }(\mathrm{mm} / \mathrm{s})$ & $\mathrm{a}$ & $\mathrm{k}$ \\
\hline & & & & \\
V1 & 32 & 2.41 & 0.86 & 1.9 \\
V2 & 26 & 1.85 & 0.95 & 1.5 \\
V3 & 25 & 2.37 & 0.77 & 1.5 \\
& & $n s^{*}$ & $n s^{*}$ & $p<0.05^{*}$ \\
A1 & 23 & 1.29 & 0.96 & 4.0 \\
A2 & 25 & 1.49 & 0.94 & 2.9 \\
A3 & 17 & 1.98 & 0.83 & 2.4 \\
\hline
\end{tabular}

* A compared to $V(n s=$ non-significant)

in venules, because platelet contribution to total blood volume is less than $0.25 \%$ (Goldsmith, 1972). Another difference between both vessel types is the presence of leukocyte rolling in venules, but not in arterioles. A major influence of this phenomenon on the velocity profile also seems unlikelly, because no correlation of the degree of leukocyte rolling with any velocity profile parameter was found. With regard to red blood cells, we have shown previously that in arterioles the shape of their velocity profile is similar to that of platelets (Tangelder et al, 1986). Local hematocrit does on the average not differ between venules and arterioles of the size used in this study (Kanzow et al, 1982; Lipowsky et al, 1980; Sarelius and Duling, 1982). However, little information is available about a possible difference in hematocrit distribution over the cross-sectional area. To which extent differences exist between venules and arterioles in other red blood cell rheological parameters, such as deformability and mutual interactions, is also not known.

The venular velocity profiles obtained in this study were also used to assess least estimates of wall shear rate and wall shear stress. Because no data points could be obtained from a layer of fluid near the wall, we interpolated the velocity profile between zero velocity at the wall (no-slip condition) and the fitted velocity of the outermost platelets. In arterioles their radial position depends mainly on the size and orientation of the platelets (Tangelder et al, 1988). By contrast, in venules we found the presence of an additional wall exclusion (see Figure 6.5). This may have lead to an underestimation of the calculated wall shear estimates in venules. As compared to a parabolic velocity distribution but with the same volume 
flow, the median shear rate was in venules 1.13 (see table 6.2) and in arterioles 2.12 (Tangelder et al, 1988) times higher. This difference was not caused by differences in platelet velocities at comparable distances from the vessel wall. This is indicated by our finding that parameter a did not differ between both vessel types (see Figure 6.5). To elucidate whether the velocity profile in the interpolated zone is indeed different between venules and arterioles, experiments are needed with flow markers which are able to approach the venular wall more closely.

In conclusion, the present study shows that in straight segments of venules, which are at least 10 vessel diameters from branch points, the velocity profiles are rather symmetric and differ significantly from those in arterioles. Adequate description requires in both vessel types a thin layer of fluid at the wall with a relatively low apparent viscosity. By contrast, in the remaining core the velocity profile differs in venules not from a parabola, but is in arterioles significantly more blunt. A relatively large wall exclusion of platelets is present in venules and not in arterioles, supporting the idea of a difference in endothelial surface characteristics (Chapter 4; Woldhuis et al, 1992).

\subsection{SUMMARY}

Distribution of velocity gradients, or shear rates in a vessel is important for interactions between blood cells and the vessel wall. We used intravital fluorescence video microscopy was used to obtain for the first time detailed information about the velocity profile in venules. Straight segments of 8 rabbit mesenteric venules (diameter 18-32 $\mu \mathrm{m}$ ), located at least 10 vessel diameters from branch points, were studied. Blood platelets, labelled in vivo with acridine red and flowing within an optical section (depth $<5$ $\mu \mathrm{m})$ about the median vessel plane, were used as natural markers of flow. Illumination with pairs of flashes yielded two images of the same cell displaced over a certain distance within the preset time interval (4-11 ms) between both flashes. All flash pairs were given at the end of the diastolic phase of the pulsatile flow. All profiles were rather symmetric. Centerline velocities ranged from 0.3 to $2.4 \mathrm{~mm} / \mathrm{s}$ (median $1.2 \mathrm{~mm} / \mathrm{s}$ ). Velocities near the wall were significantly higher than expected in case of a parabolic profile, indicating the existence of a thin layer of fluid at the wall with reduced viscosity. In the remaining core, the shape of the profile did not differ from that of a parabola, in contrast to arterioles of similar size in which it is more blunt, as found previously. Consequently, the ratio of 
maximal and mean velocity, which is 2 for a parabola and 1 for complete plug flow, is significantly larger in venules (range: 1.62-2.13; median 1.7) than in arterioles (median: 1.5). This discrepancy cannot be explained by differences in centerline velocity. Least estimates of wall shear rate obtained from these profiles did not differ significantly from those based on a parabola, but are probably underestimated because we found in venules a relatively large wall exclusion of platelets. This exclusion was absent in arterioles, suggesting a difference in endothelial surface characteristics. 
$\therefore$ 


\section{PLATELET FÅHRAEUS EFFECT IN ARTERIOLES AND VENULES}

\subsection{INTRODUCTION}

In the microcirculation, the concentration of blood cells is not uniformover the microvessel cross-section. Red blood cells preferentially flow in the areas with higher flow velocities, i.e. in the vessel center. This results in a mean red blood cell velocity that is higher than mean plasma velocity and, hence, than mean whole blood velocity. The consequence of this difference in mean velocities is that the average residence time of red blood cells in a certain vessel segment is reduced as compared to the mean residence time of whole blood. Consequently, the local red blood cell concentration in the vessel has to be lower than for homogeneously distributed red blood cells, because otherwise an accumulation of red blood cells would occur within the microcirculation. This so-called Fåhraeus effect, i.e. the reduction of local vessel hematocrit due to differences in mean travelling speed between red blood cells and the suspending solution (Fåhraeus, 1928, 1929), is flow-dependent (Gaehtgens et al, 1978). It also depends on vessel diameter, and occurs progressively in vessels smaller than approximately $100 \mu \mathrm{m}$, being strongest in vessels of about $10 \mu \mathrm{m}$ in diameter (Albrecht et al, 1979).

The principle of the Fåhraeus effect also applies to blood platelets. Opposite to red blood cells, in arterioles more platelets are flowing near the wall than in the vessel center (Tangelder et al, 1986; chapter 4; Woldhuis et al, 1992). Hence, one expects their mean velocity to be reduced. Therefore, one would intuitively conclude that an inverted Fahraeus effect might occur. This then would lead to a mean platelet velocity that is lower than the mean blood velocity, and, hence, to an increased platelet concentration in these vessels. In venules, however, the concentration distributions differ distinctly from those in arterioles, with higher concentrations in the vessel center. In addition, recent experiments showed that also the velocity profiles differ in

\footnotetext{
Submitted for publication
} 
arterioles and venules. In venules, the velocity profiles are less blunt (chapter 6; Woldhuis et al, submitted) than in arterioles (Tangelder et al, 1986).

In the present paper, we combined the data of all detailed concentration distributions with velocity profiles measured in arterioles and venules of anesthetized rabbits, to obtain an estimate of the platelet Făhraeus-effect. All vessels were located in the mesentery, ranging in diameter from 17 to $33 \mu \mathrm{m}$. In addition, the same calculations were performed assuming a parabolic velocity profile in these vessels.

\subsection{METHODS}

Calculations were applied to data obtained from experiments performed for other studies (Tangelder et al, 1986; chapter 4; Woldhuis et al, 1992; chapter 6; Woldhuis et al, submitted). The experimental methods have been described in detail in those articles. In short, in anesthetized rabbits a segment of the distal illeum was brought outside for intravital video microscopy. Circulating platelets were visualized with epi-fluorescence microscopy after intravenous injection of the fluorescent dye acridine red. Flashes of short duration were given to avoid smearing of the image of the platelets (Tangelder et al, 1985).

In the concentration distribution experiments, a single flash was given in the blanking period of the video camera, every 6 th or 8 th TV field, thus preventing that a platelet would be counted twice. In the velocity profile experiments pairs of flashes were given with a short, preset time interval between both flashes (range: $2-11 \mathrm{~ms}$ ), provoking two images of the same platelet displaced over a certain distance. In all experiments flash pairs were given at the moment in the cardiac cycle that flow velocity in the microvessel was at its lowest level (diastolic phase). Analysis was performed off-line on still video fields. Only platelets flowing within a thin optical section with a depth of approximately $5 \mu \mathrm{m}$ and located about the median vessel plane were used for analysis (Tangelder et al, 1982b). In all cases data were obtained from vessel segments without branch points within at least 10 vessell diameters. Curves immediately up-or downstream were avoided.

During off-line analysis, the centroid (center of gravity) of the image of a platelet was marked. The distance (d) between the platelet centroid and the left vessel wall (looking downstream), as well as the local vessel diameter (2R) were measured, and used to calculate its relative position $(d / 2 R)$ across the vessel diameter. For the velocity measurements this was 
done for both images of a platelet. In this case its relative position was taken as the mean of the relative positions of both images. Its displacement was measured directly as the distance between the two centroids of the same platelet. From the displacement and the given time interval the velocity was calculated.

In the concentration distribution experiments, for each vessel we grouped the platelet counts in 20 equal segments across the vessel diameter. The histograms of platelet counts were then converted to radial distributions of platelet counts, consisting of 10 segments, by pooling the data of the left and the right hand sides of the vessels $(C(r)$; with $r=\rho / R ; \rho$ is the distance to vessel center; $0 \leq r \leq 1)$. The resulting histograms were converted to normalized radial concentration distributions by normalizing them with respect to the mean concentration over the whole cross-section of that vessel (chapter 4; Woldhuis et al, 1992; see appendix). Figure 7.1 shows the average normalized radial concentration distributions in arterioles and venules (chapter 4; Woldhuis et al, 1992).

In the velocity profile experiments, the data were fitted with the equation:

$$
V(r)=V_{\max }\left(1-|a \cdot r|^{k}\right)
$$

where $V_{\max }$ is the velocity in the center of the vessel; parameter a is a scaling factor, allowing a non-zero intercept of the fit with the vessel wall ( $a>0$; see below) and $\mathrm{k}$ is a factor describing a possible flattening of the profile within the fitted part (see Tangelder et al, 1986 for details). The vertical stripes denote absolute values. All profiles measured were rather symmetric, in both arterioles and venules, with the top of the profile positioned close to the vessel center. Therefore, fits were performed with symmetric profiles. The velocity profile parameters used for both vessel types are shown in table 7.1. In principal, fits apply only to that part of the vessel from which data could be collected. Parameter a actually denotes the part of the completely fitted profile, i.e. from $V_{\max }$ to zero, that can be contained within the vessel; a parameter a value of 0.9 , for example means that only $90 \%$ of the completely fitted profile can in reality be contained within the vessel. Parameter $k$, which estimates blunting of the profile in the core fluid, was significantly greater in arterioles than in venules $(p<0.005)$. Parameter a did not differ significantly between arterioles and venules.

For further calculations the velocity profiles were normalized to a 
mean value of 1 :

$$
\operatorname{Norm} V(x)=\frac{(k+2)}{\left(k+2-2 a^{k}\right)}\left(1-a^{k} Y^{k}\right)
$$

NORMV(r) is normalized velocity; see appendix. Figure 7.2 shows the average normalized velocity profiles for arterioles and venules in a one-fluid and a two-fluid model (Tangelder et al, 1986; chapter 6; Woldhuis et al, submitted).

Using in this way the blood platelets as natural markers of flow, we never measured velocity values at positions closer to the wall than 0.04 to 0.11 radial units for the various arterioles and 0.10 to 0.21 for the venules. In one set of calculations the fitted profiles were assumed to extend completely to the vessel wall (one fluid-model). In a second set of calculations, fits were only used from the center to the measurement points closest to the wall: the transition point. The transition point was defined as the mean radial position of the six platelet centroids ( 3 from left and 3 from right hand side) closest to the wall (Tangelder et al, 1988). Since the velocity at the wall has to be zero to satisfy the no-slip condition (Caro et al, 1978), we inferred the existence of a thin layer of fluid between the transition point and the wall with properties different from those in the core (two-fluid model). We assumed a linear velocity profile between the transition point and the wall, as we did before when we calculated the wall shear rates in these vessels (Tangelder et al, 1988). The error made by this simplification
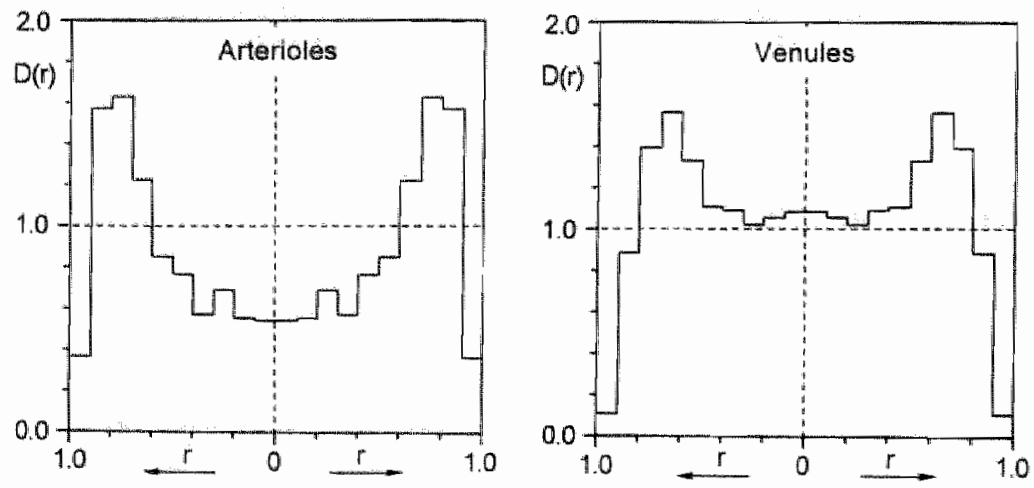

Figure 7.1: Mean relative radial platelet concentration distributions $(D(r)$, normalized to a mean value of I) in arterioles and venules. Data from Woldhuis et al, 1992. 

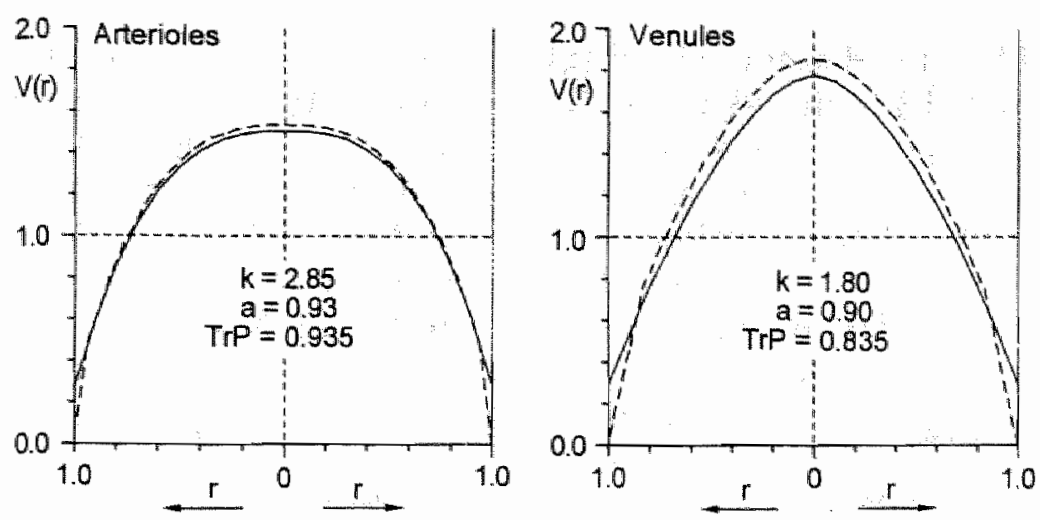

Figure 7.2: Median velocity profiles ( $V(r)$, normalized to a mean velocity of 1$)$ for arterioles and venules. Solid lines indicate one-fluid model and dashed lines two-fluid model. Line at $V=1$ indicates mean velocity within vessel. Data from Tangelder et al, 1986 and Woldhuis et al, in press.

over a parabolic profile in the transition layer was in all cases less than $4 \%$. The normalization procedure (see appendix) was also applied to this twofluid description of the velocity profile.

The arterioles in which the velocity experiments have been performed ranged in diameter from 17 to $32 \mu \mathrm{m}$ (median: $25 \mu \mathrm{m}$ ); their maximal velocities ranged from 1.3 to $14.4 \mathrm{~mm} / \mathrm{s}$ (median: $3.4 \mathrm{~mm} / \mathrm{s}$ ). In the venules used for the velocity experiments the diameters ranged from 18 to $32 \mu \mathrm{m}$ (median: $25 \mu \mathrm{m}$ ); their maximal velocities ranged from 0.29 to $2.41 \mathrm{~mm} / \mathrm{s}$ (median: $1.18 \mathrm{~mm} / \mathrm{s}$ ). The median diameters of the arterioles and venules used in the concentration distribution experiments were $24 \mu \mathrm{m}$ (range: 17-33 $\mu \mathrm{m}$ ) and $23 \mu \mathrm{m}$ (range: $18-31 \mu \mathrm{m}$ ), respectively. The diameters of the arterioles and venules used in the present calculations did not differ significantly.

\section{Calculation of mean platelet velocity}

Mean platelet velocity (MPV) was calculated with the following equation:

$$
M P V=\frac{\iint N O R M V(I) \cdot D(I) \cdot d A}{\iint d A}
$$


where NORMV(r) is the normalized velocity (see above) as a function of the relative radial position. $D(r)$ is the normalized radial concentration distribution and integral $\mathrm{dA}$ denotes the surface integral over the crosssectional area of the vessel (see appendix for detailed information). Due to the normalization of the individual velocity profiles and the radial concentration distributions, the mean platelet velocity values are normalized to a value of 1 in case of a homogeneous platelet distribution (i.e. $D=1$ for all r), in which case mean platelet velocity equals mean whole blood velocity.

To allow assessment of the influence of variations in shape of both the concentration distribution and the velocity profile, we used the following combinations. For arterioles, calculations were performed by combining 12 measured velocity profiles (Table 7.1; Tangelder et al, 1986) and the mean concentration distribution for arterioles as determined in a different set of experiments (chapter 4; Woldhuis et al, 1992). In addition, an assumed parabolic velocity profile with this same mean velocity was also combined

Table 7.1: Platelet velocity profile parameters as determined in arterioles (A; Tangelder et al, 1986) and in venules (V; Woldhuis et al, submitted). Vessels indicated by serial number which is also used in table 7.2. $D$ and $S$ indicate that the profiles were determined during the diastolic or systolic flow phase in the microvessel, respectively.

\begin{tabular}{llllll}
\hline $\mathrm{A}$ & $\mathrm{k}$ & $\mathrm{a}$ & $\mathrm{V}$ & $\mathrm{k}$ & $\mathrm{a}$ \\
\hline 1 D & 2.4 & 0.83 & 1D & 1.4 & 0.89 \\
2 D & 3.2 & 0.93 & 2D & 2.3 & 0.93 \\
3 D & 4.0 & 0.96 & 3D & 2.2 & 0.91 \\
4 D & 2.6 & 0.90 & 4D & 2.6 & 1.00 \\
5 D & 2.7 & 0.86 & 5D & 1.5 & 0.77 \\
6 D & 2.9 & 0.94 & 6D & 1.5 & 0.95 \\
7 D & 2.9 & 0.92 & $7 \mathrm{D}$ & 1.7 & 0.86 \\
8 D & 2.8 & 0.93 & $8 \mathrm{D}$ & 1.9 & 0.86 \\
9 D & 3.0 & 0.95 & & & \\
10S & 2.3 & 0.87 & & & \\
11S & 2.7 & 0.93 & & & \\
12S & 3.0 & 0.95 & & & \\
\hline med & 2.85 & 0.93 & med & 1.8 & 0.90 \\
par & 2.0 & 1.00 & par & 2.0 & 1.00 \\
\hline
\end{tabular}

med $=$ median velocity profile; par $=$ parabolic velocity profile.

$\mathrm{k}$ is power of fit for velocity profile; $\mathrm{a}$ is a scaling factor. 
with the mean concentration distribution for arterioles. Moreover, we combined the individual concentration distributions of 9 arterioles (chapter 4; Woldhuis et al, 1992) and the median velocity profile as measured in the other set of experiments (Table 7.1; Tangelder et al, 1986). These 9 arteriolar concentration distributions were also combined with an assumed parabolic velocity profile.

For venules, a similar scheme was followed for 8 velocity profiles (table 7.1; chapter 6; Woldhuis et al, submitted) and 10 concentration distributions (chapter 4; Woldhuis et al, 1992).

\section{Statistics}

Comparisons of the MPV as calculated for arterioles and venules with MPV in case of a homogeneous platelet distribution (MPV $=1$ ), and comparison of MPV without and with transition point (one versus two fluid model) were performed with the paired Wilcoxon-test (SPSS-PC statistical package, SPSS Inc., Chicago, USA). In the first case, this test was used as non-parametric alternative to the one sample t-test (Bradley, 1968), Comparisons of data between arterioles and venules were made using the Mann-Whitney U-test (SPSS-PC statistical package). When the p-value was equal to or exceeding 0.05 , a difference was considered as statistically nonsignificant.

\subsection{RESULTS}

The various steps of the calculations can be demonstrated, using as an example for arterioles their median normalized radial platelet concentration distribution combined with their median velocity profile (see Figure 7.3). The normalized radial platelet concentration distribution was obtained from 10 segments within the optical section, after pooling left and right hand sides (Figure 7.3A). For each of these 10 segments, a ring exists in which the concentration is the same as in the concomitant segment (Figure 7.3A). Each ring has a different surface area (Figure 7.3B). Consequently, the platelet concentration (Dr) in each ring, or segment (Figure 7.3C), contributes to a varying extent to the mean platelet concentration in the vessel as a whole (Figure 7.3D). Similarly, the velocities of the fluid elements at the various positions across the vessel radius (Figure 7.3E), contribute to varying extent to the mean velocity in the vessel as a whole, leading to the velocity surface-integral histogram (Figure 7.3F). 

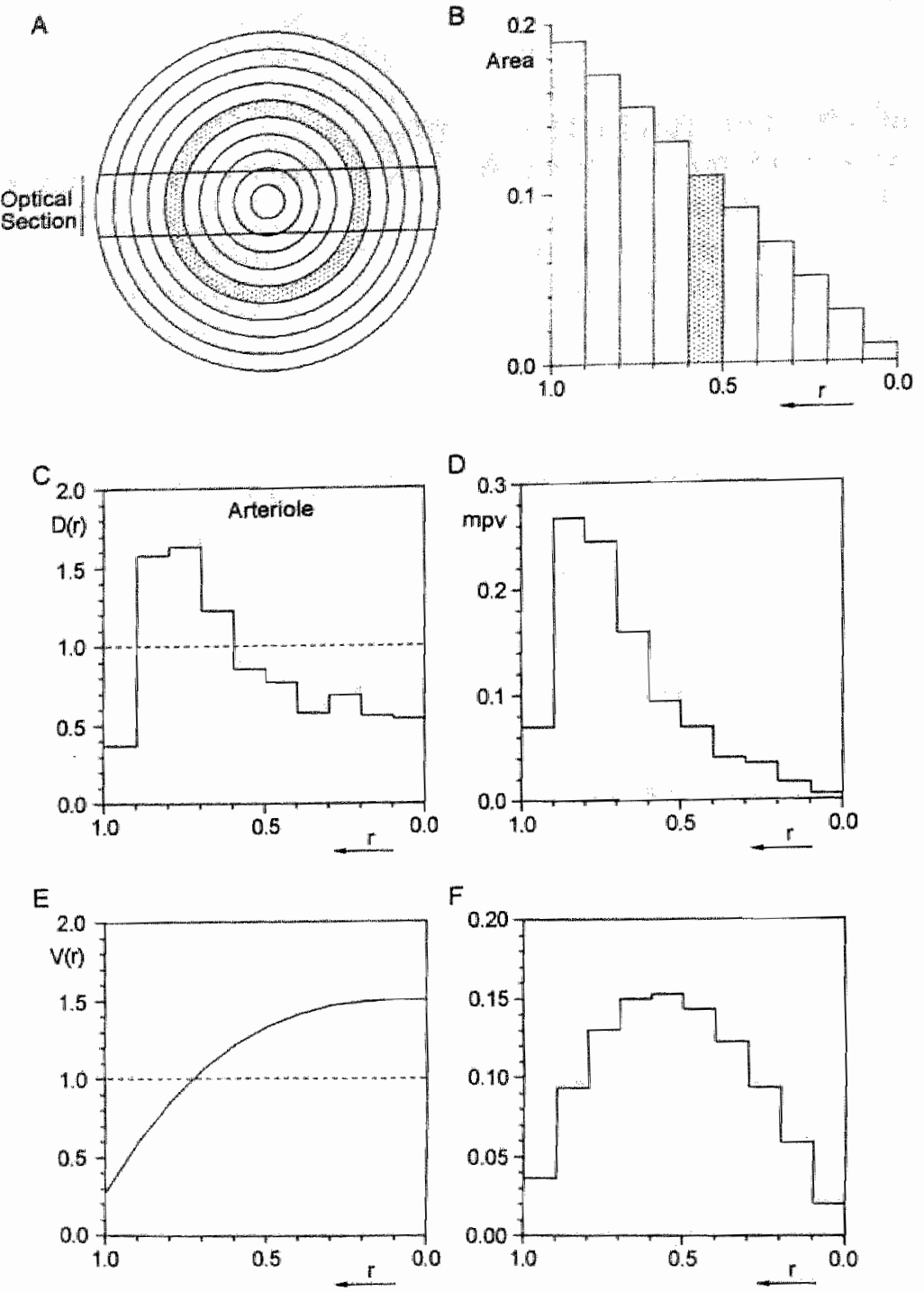

Figure 7.3: Illustration of the various steps of the calculations to obtain the mean platelet velocity. A) Ten radial segments with concomitant rings of same platelet concentration. B) Histogram of the surface area of the various rings. C) The median nomalized radial platelet concentration distribution in the arterioles, used as an example. The line at a level of 1 indicates the mean concentration. D) The contribution of the various segments to the mean platelet concentration. E) Median normalized arteriolar velocity profile F) Contributions of the various rings to the mean velocity of the velocity profile. 


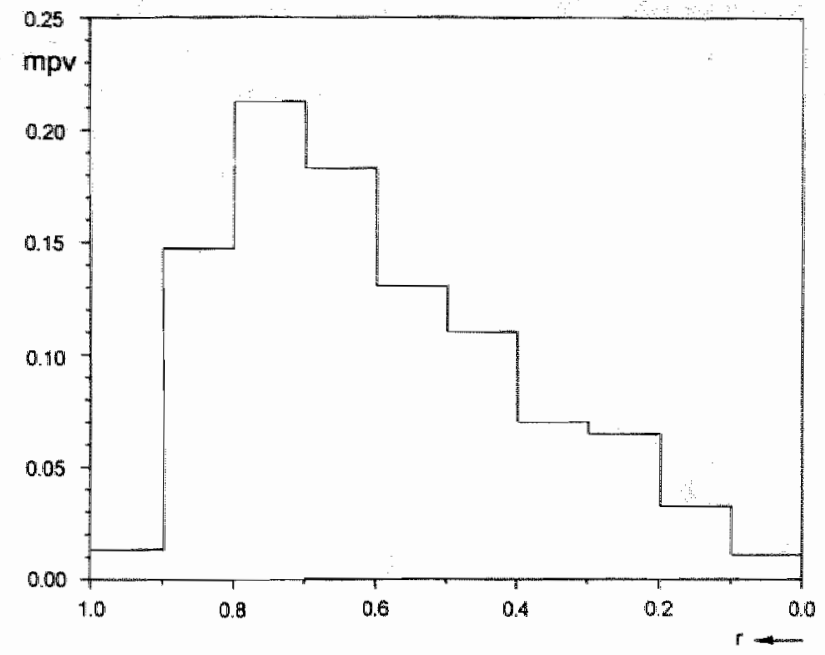

Figure 7.4: Contribution of the various rings to mean platelet velocity (MPV). which is set at unity.

The contribution of each segment to the mean platelet velocity (Figure 7.4) is obtained by multiplication of the radial platelet concentration histogram (Figure 7.3C) and the velocity surface-integral histogram (Figure 7.3F).

The mean platelet velocities (MPVs) which were calculated for the individually measured velocity profiles are presented in table 7.2. Under MPV two columns are presented, one without (no-TrP) and one with accommodation to the no-slip condition (TrP). This latter column demonstrates the influence of a transition layer near the wall (two-fluid model). At the bottom of the table also data for a parabolic profile combined with the mean platelet concentration distribution are shown. Values of the relative radial positions of the transition points (column TrP) are presented as well. In case of a one-fluid model (columns MPV no-TrP), the mean platelet velocities for arterioles ranged from 0.99 to 1.02 , with a median of 1.00 ; i.e. not significantly different from the MPV in case of a homogeneous platelet distribution. The arteriolar mean platelet velocity for the parabolic profile was 0.97 . For venules, the mean platelet velocities without adaptation to a two-fluid model varied from 1.08 to 1.18 with a median of 1.12. This is significantly higher $(p<0.01)$ than expected in case of a homogeneous platelet distribution. The venular MPV value for the parabolic profile is even higher: 1.18 . 
Table 7.2: Mean platelet velocities (MPV) of arterioles (A) and verules (N) for individual velocity profiles; without (no-TrP) and with transition point (TrP), combined with the arteriolar or venular median concentration distributions. Velocity profiles with TrP satisfy the no-slip condition at the vessel wall. In addition the latter were also combined with a parabola (see also figure 1). The relative radial position of the transition point (TrP) is shown as well.

\begin{tabular}{|c|c|c|c|c|c|c|c|}
\hline \multirow[t]{2}{*}{ A } & \multicolumn{2}{|c|}{ MPV } & \multirow[t]{2}{*}{$\operatorname{TrP}$} & \multirow[t]{2}{*}{$\mathrm{V}$} & \multicolumn{2}{|c|}{ MPV } & \multirow[t]{2}{*}{$\operatorname{Tr} P$} \\
\hline & No-TrP & $T r P$ & & & No-TrP & $\operatorname{Tr} P$ & \\
\hline $1 \mathrm{D}$ & 0.99 & 1.01 & 0.94 & $1 D$ & 1.12 & 1.18 & 0.79 \\
\hline $2 \mathrm{D}$ & 1.01 & 1.02 & 0.93 & $2 \mathrm{D}$ & 1.13 & 1.17 & 0.82 \\
\hline $3 D$ & 1.02 & 1.03 & 0.89 & $3 \mathrm{D}$ & 1.12 & 1.17 & 0.82 \\
\hline $4 \mathrm{D}$ & 1.00 & 1.01 & 0.94 & $4 \mathrm{D}$ & 0.84 & 1.18 & 0.84 \\
\hline $5 \mathrm{D}$ & 1.00 & 1.01 & 0.96 & SD & 1.08 & 1.14 & 0.90 \\
\hline $6 \mathrm{D}$ & 1.00 & 1.01 & 0.94 & $6 \mathrm{D}$ & 1.15 & 1.17 & 0.83 \\
\hline $7 \mathrm{D}$ & 1.00 & 1.01 & 0.95 & $7 \mathrm{D}$ & 1.11 & 1.17 & 0.84 \\
\hline $8 \mathrm{D}$ & 1.00 & 1.01 & 0.93 & $8 \mathrm{D}$ & 1.11 & 1.15 & 0.90 \\
\hline $9 \mathrm{D}$ & 1.00 & 1.01 & 0.92 & & & & \\
\hline $10 \mathrm{~S}$ & 0.99 & 1.01 & 0.93 & & & & \\
\hline $11 \mathrm{~S}$ & 1.00 & 1.01 & 0.93 & & & & \\
\hline $12 S$ & 1.00 & 1.01 & 0.95 & & & & \\
\hline med & 1.00 & 1.01 & 0.935 & med & 1.12 & 1.17 & 0.83 \\
\hline par & 0.97 & & & par & 1.18 & & \\
\hline
\end{tabular}

D: diastole. S: systole, med = median velocity profile; par = parabolic velocity profile. Median concentration distributions obtained from Woldhuis et al, 1992.

The adaptation of the profiles with a thin fluid layer at the wall with altered properties to satisfy the no-slip condition, led to small but significant increases of mean platelet velocity (columns MPV TrP in table 7.2). In all arterioles the increase was less than $2 \%(\mathrm{p}<0.001)$. In venules, the increase was always less than $6 \%(\mathrm{p}<0.001)$. Adaptation of a two-fluid model also reduced the differences in MPV between the various vessels.

The data for the individual concentration distributions combined with the median velocity profiles, both with and without adaptation to satisfy the no-slip condition, are presented in table 7.3. The variation in these data is considerable in both arterioles and venules. The results for arterioles range from 0.77 to 1.09 , with a median of 1.04 . Arterioles $C$ and $D$ have mean platelet velocities of less than 1 , and stray clearly from the other vessels that all have mean platelet velocities above 1 . In venules, the variation is less: $1.03-1.25$, with a median of 1.12 and without clear stray values. Again the 
influence of the transition layer was limited, with the influence in venules (less than 5\%; $\mathrm{p}<0.001$ ) being larger than in arterioles (less than $2 \%$; $\mathrm{p}<0.001)$.

\subsection{DISCUSSION}

The flowing of platelets in arterioles at higher concentrations near the vessel wall than in the center, did not result in an overvelocity of platelets as compared to a homogeneous platelet distribution. Because on the average each cell or fluid element travelling at the same radial position will do so with the same velocity, as has been demonstrated for platelets and red blood cells (Tangelder et al, 1986), mean platelet velocity in case of a homogeneous platelet distribution will equal mean whole blood velocity. Hence, in arterioles the actual mean platelet velocity did not differ from mean whole blood velocity. Therefore, no Fåhraeus effect is present for platelets in arterioles: local platelet concentration in these vessel segments is not changed by the rheological conditions. In venules, on the contrary, the presence of a relative concentration peak more distant from the wall in

Table 7.3: Mean platelet velocities (MPV) for individual concentration distributions and the median velocity profile (see table 7.2$)$ in arterioles $(A)$ or venules $(V)$, without $(N o-T r P)$ and with transition points (TrP) in the velocity profiles. In the latter case the velocity profiles satisfy the no-slip condition at the vessel wall. For median values of $\operatorname{Tr} P$ see table 7.2.

\begin{tabular}{llllll}
\hline \hline A & \multicolumn{2}{c}{ MPV } & V & \multicolumn{2}{c}{ MPV } \\
& No-TrP & TrP & & No-TrP & TrP \\
\hline A & 1.04 & 1.05 & A & 1.11 & 1.15 \\
B & 1.09 & 1.11 & B & 1.14 & 1.19 \\
C & 0.91 & 0.91 & $\mathrm{C}$ & 1.22 & 1.27 \\
D & 0.77 & 0.76 & $\mathrm{D}$ & 1.03 & 1.06 \\
E & 1.04 & 1.06 & E & 1.04 & 1.08 \\
F & 1.04 & 1.06 & F & 1.17 & 1.22 \\
G & 1.03 & 1.05 & G & 1.25 & 1.30 \\
H & 1.06 & 1.07 & H & 1.12 & 1.17 \\
J & 1.02 & 1.04 & J & 1.11 & 1.14 \\
& & & K & 1.08 & 1.11 \\
\hline median & 1.04 & 1.06 & median & 1.12 & 1.16 \\
\hline
\end{tabular}

Concentration distributions obtained from Woldhuis et al, 1992. 
combination with a relatively high concentration in the vessel center, resulted in an increased mean velocity for platelets as compared to mean whole blood velocity. Hence, in venules a Fåhraeus effect for platelets is present and will result in a reduced platellet concentration as compared to the systemic blood concentration of platelets.

The considerable variations in the velocity profile parameters in arterioles, with parameter $\mathrm{k}$ ranging from 2.3 to 4.0 , and parameter a from 0.83 to 0.96 , did not result in significant changes in the platelet Fåhraeus effect (see table 7.2). This is due to the interaction between parameters $\mathrm{k}$ and $a$, and also to the actual position of the peak in the platelet concentration distribution (see Figure 7.1). At the location of the peak in platelet concentration, the velocities happen to be near the mean velocity of the profile (see Figure 7.5A). Hence, the presence of a concentration peak at this position can hardly result in a change of the mean platelet velocity. This explains why no net platelet Fåhraeus effect is present in arterioles. Figure $7.5 \mathrm{~A}$ also shows the effect in arterioles of a hypothetical parabolic velocity profile. For a parabola the radial position at which the velocity is equal to the mean velocity of the profile, is located slightly more towards the center of the vessel. Hence, a parabolic velocity profile might result in a minor inverted platelet Fåhraeus effect, as indicated by table 7.2.

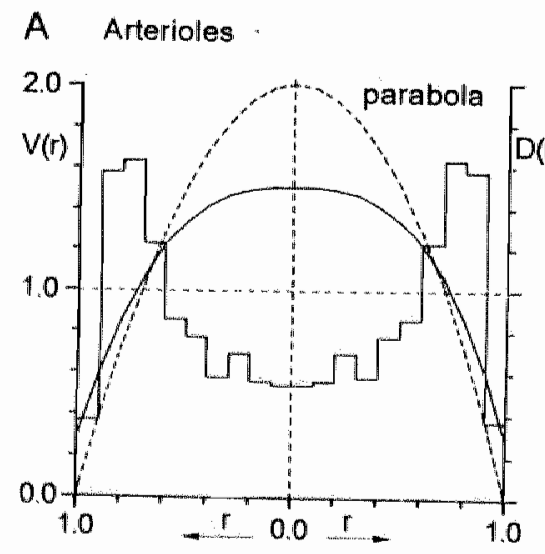

$B$ Venules

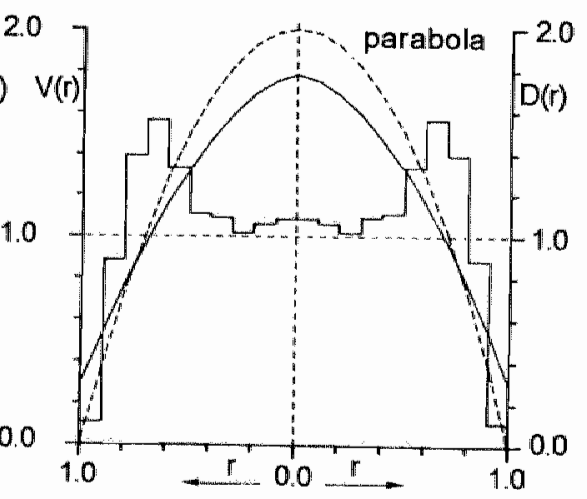

Figure 7.5: Mean relative radial platelet concentration distribution and median velocity profile of arterioles $(A)$ and venules $(B)$. Both are superimposed to demonstrate the relative position of the concentration peak with regard to the radial position at which the velocity is equal to the mean velocity of the profile. Parabolic profile (in dashed line) shown for
comparison. 
This would implicate a slight decrease in mean platelet velocity leading to a small increase in platelet concentration in the microvessel as compared to systemic platelet concentration.

In venules, the variations in the velocity profile parameters were even more pronounced than in arterioles: parameter $\mathrm{k}$ ranged from 1.4 to 2.6 , and parameter a from 0.77 to 1.0 . Despite this variation in velocity profile parameters, mean platelet velocities showed little variation. This can be explained by an interaction between parameters $\mathrm{k}$ and $\mathrm{a}$, and the location of the peak in the platelet concentration distribution. This peak is in venules located more towards the vessel center and is present at a radial position, where velocities are already above the mean velocity of the profile (see Figure $7.5 \mathrm{~B}$ ). This in combination with a relatively high concentration in the vessel center, explains the presence of a Fåhraeus effect for platelets in venules. For the hypothetical parabolic profile, the radial position, where velocity equals mean velocity of the profile, is located closer to the vessel wall (see Figure 7.5B), in contrast to the situation in arterioles (Figure $7.5 \mathrm{~A})$. Hence, in venules the platelet Fahhraeus effect in this case is even stronger than that for the actually measured velocity profiles.

In a separate set of calculations we adapted the fitted velocity profiles to the no-slip condition taking into account also the presence of the thin fluid layer between transition point and vessel wall. This did not lead to considerable changes in mean platelet velocity. This thin fluid layer near the wall represented between 8 and $22 \%$ of the cross-sectional area in arterioles, and between 19 and $38 \%$ in venules. Despite these values, the influence on the platelet Fåhraeus effect of inclusion of this layer into the calculations is limited. This is due to the limited number of platelets present in these outer radial segments. It is interesting to note that in venules application of the two-fluid model led to a strong reduction in variability of the calculated mean platelet velocity (see table 7.2).

The variations in the concentration distributions, however, had a considerable influence on the mean platelet velocities, as can be seen in table 7.3. In venules, the mean platelet velocities ranged from 1.03 to 1.25 . Inspection of the venular concentration distributions showed that the peak of the concentration distributions varied in position, but was always closer to the vessel center than to the site in the vessel where the velocity of the profile equals mean velocity. This explains why clear stray values where absent in venules. By contrast, for the various concentration distributions in arterioles, 7 out of 9 distributions yielded a mean platelet velocity between 1.02 and 1.09 , but two arterioles exhibited the initially anticipated inverted Fåhraeus effect with mean platelet velocities of 0.77 and 0.91 , respectively. 
A comparison of the various concentration distributions showed that in these two arterioles the peaks were located closer to the wall than in all other arterioles. This is illustrated in Figure 7.6 for the arteriole with the mean platelet velocity of 0.77 . Therefore, the concentration and, hence, the number of platelets between the relative radial positions 0.8 and 1 , which comprises $36 \%$ of the total cross sectional surface area (see Figure 7.3B), was much higher than in the other 7 vessels. However, the velocities in that radial section were below mean (see Figure 7.6). Consequently, an inverted platelet Fåhraeus effect was calculated for these two vessels. The fact that the intuitive interpretation of a possible inverted Fåhraeus effect in arterioles was only correct in these two cases but not in the other 7, indicates the crucial importance to consider the location of the peaks and troughs relative to the position where the value of the velocity profile is actually equal to the mean velocity in the vessel.

The data for the present calculations were obtained from an optical section of approximately $5 \mu \mathrm{m}$ thickness about the median vessel plane. In the center of the microvessels, this means that the actual distance of the platelet to the vessel center may have been off by about 0.2 radial units. This possible error is due to the fact that the platelets may actually have been this distance above or below the median plane of the vessel. By contrast, near the wall the influence of this uncertainty in actual radial position becomes negligible. Despite the fact that in the vessel center the shapes of the velocity profile and the concentration distribution are not very accurate, corrections for these inaccuracies would have changed relatively little in the final outcome. This is due to the small surface area belonging to that part of the vessels. Hence, the calculated platelet Fåhraeus effects are a reliable approximation.

Based on the calculated Fåhraeus effect, it seems that no significant changes occur in the local platelet concentrations in mesenteric arterioles of rabbits. However, besides the Fåhraeus effect that is based on the velocity profile and the concentration distribution (tube Fåhraeus effect), the separation of phases at bifurcations leading to a network Fåhraeus effect (Pries et al, 1986) should also be taken into account. Tangelder (1982) demonstrated that in arterioles, where the platelets flow preferentially near the wall, directly past a bifurcation the relative platelet concentration peak on the side of the bifurcation has disappeared. A disproportional number of platelets has entered the side branch. Due to this skimming effect of platelets in arterioles, a nonhomogeneous distribution of the platelets within a network may result (Ofjord and Clausen, 1986). Our data, however, do not allow for an analysis of this network platelet Fåhraeus effect. This is because 


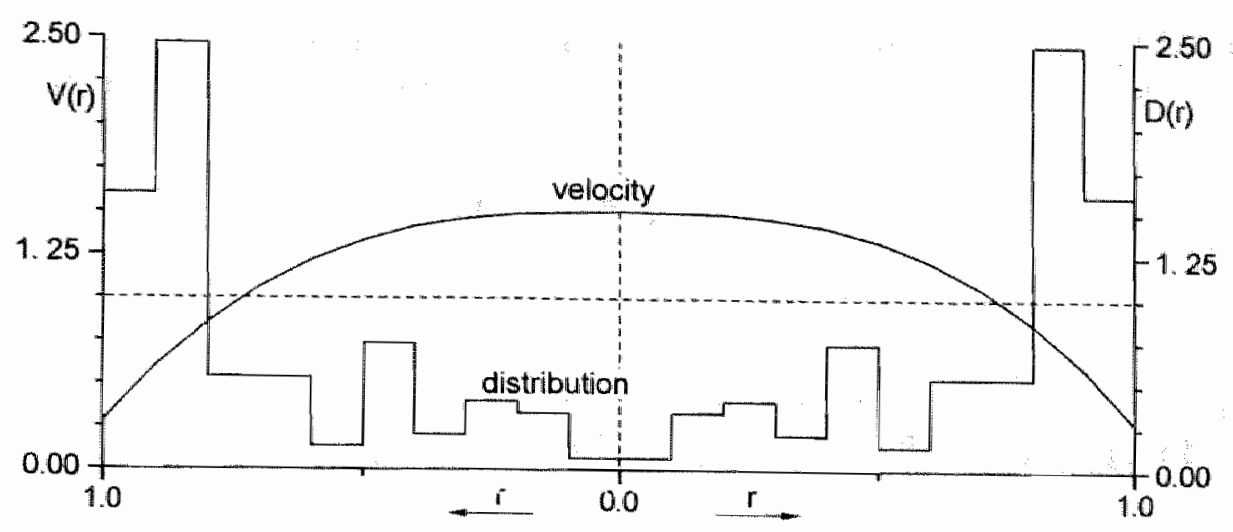

Figure 7.6: Relative radial platelet concentration distributions of arteriole $D$ in table 7.3, which exhibited a strong inverted Fahraeus effect. Superimposed is the median velocity profile. Note that the peak of the relative radial concentration distribution of this arteriole is closer to the wall than the peak of the median relative radial concentration distribution as shown in Fig 7.1. As shown in the Figure, it is also closer to the wall than the position where the velocity profile crosses its mean value of 1 (indicated by dashed line).

we only measured platelet concentration distributions relative to their own mean value. Therefore, they cannot be used to calculate the actual concentration in that particular microvessel. Moreover, the actual thickness of the optical section was unknown, being estimated to be about $5 \mu \mathrm{m}$ or less (Tangelder et al, 1982b). This latter factor precluded accurate assessment of the true platelet concentration in these microvessels.

In conclusion: calculations demonstrate that in arterioles without nearby upstream and downstream bifurcations or curves the governing rheological conditions do not induce a net platelet Fåhraeus effect. by contrast, in venules, a higher mean velocity for platelets than for whole blood was found to result in a platelet Fåhraeus effect and, hence, in a reduced mean local platelet concentration. When predicting the influence of a peak in cell concentration on a possible Fåhraeus effect, one should consider the position of the concentration peak relative to the radial position where the velocity profile attains the value of its mean velocity, rather than the mere fact that the peak is located away from the center. 


\subsection{APPENDIX}

Normalization of any function over the vessel cross-section

For any given function $f(r)$, the mean over the whole cross-sectional area of a microvessel $\left(f_{\text {mean }}\right)$ is given by:

$$
f_{\text {mean }}=\frac{\iint f(I) \cdot d A}{\iint d A}
$$

where integral dA denotes the surface integral over the whole crosssectional area of the vessel. Normalization of $f(r)$ is accomplished by dividing $f(r)$ by its mean value $\left(f_{\text {mean }}\right)$ :

$$
F(I)=\frac{f(r)}{f_{\text {miran }}}
$$

where $F(r)$ is the normalized equivalent of $f(r)$. This means that the normalized function $F(r)$ has a mean of 1 when calculated over the whole vessel cross-sectional area.

\section{Calculation of relative radial concentration distribution}

The histogram of the radial distribution of platelet counts can be considered as a radial distribution of the platelet concentration in arbitrary units: $C(r)$. The normalized radial concentration distribution $D(r)$ is easily found:

$$
D(r)=\frac{C(r)}{C_{\text {mean }}}
$$

where $\mathrm{C}_{\text {mean }}$ is the mean value of the platelet count distribution over the whole cross-section of that vessel (chapter 4; Woldhuis et al, 1992).

\section{Calculation of the normalized velocity profiles}

The measured velocity profiles were fitted with equation 1 (see methods). $V(r)$ is de velocity at radial position $r$. The mean velocity profile $\left(\mathrm{V}_{\text {mean }}\right)$ is defined by: 


$$
V_{\text {mean }}=\frac{\iint V(r) \cdot d A}{\iint d A}
$$

The normalized velocity profile NORMV(r), is defined by:

$$
\operatorname{NORMV}(r)=\frac{V(r)}{V_{\text {mean }}}
$$

In case the fitted profile is used from the vessel center $(r=0)$ up to the vessel wall $(r=1)$, one can show that (see Tangelder et al, 1986):

$$
V_{\text {mean }}=V_{\max } \frac{k+2-2 a^{k}}{k+2}
$$

where $V_{\text {MAX }}$, a and $\mathrm{k}$ are parameters of equation 1 (see methods). In the case in which we included a thin layer between transition point and the vessel wall, with a linear velocity gradient to satisfy the no-slip condition, the normalization did not yield a simple expression.

\subsection{SUMMARY}

Calculations were performed to estimate a possible Fåhraeus effect for platelets flowing in arterioles and venules of rabbit mesentery. Data from our detailed concentration distributions (Woldhuis et al, 1992) were combined with those of velocity profiles (Tangelder et al, 1986; Woldhuis et al, in press) to calculate mean platelet velocity. Diameters of the vessels from which the data were taken ranged from 17 to $33 \mu \mathrm{m}$. In arterioles the governing rheological conditions did not induce a change in mean platelet velocity, as compared to mean whole blood velocity. Hence, no net platelet Fåhraeus effect occurred. In venules by contrast, a higher mean velocity was found for platelets than for whole blood. In one set of calculations the fit was assumed to extent to the wall (one fluid model). In a second set of calculations, the fits were taken for the core of the vessels only ( central 76 to $96 \%$ by radius), and a thin layer of fluid was inferred art the wall (two 
fluid model). A linear velocity profile was assumed in this layer, which induces an error of maximally $4 \%$ in this estimation of the locall velocity. In arterioles, the differences between the one and the two fluid model were negligible, in venules, the two fluid model, which satisfies the no-slip condition at the wall, yielded mean platelet velocities that were 3-5\% higher than for the one fluid model. When predicting the influence of a peak in cell concentration, one should consider the position of the concentration peak relative to the radial position where the velocity profile attains the value of its mean velocity rather than the mere fact that the peak is located away from the center. 


\section{GENERAL DISCUSSION}

In this thesis several studies were performed to investigate in vivo the rheological behavior of blood platelets in microvessels. The preparation used for these studies, the rabbit mesentery, was selected, because of its thin and transparent structure. Individual platelets flowing in small mesenteric microvessels can be observed directly using intravital fluorescence microscopy. An additional advantage of this tissue for our studies was that flow and microvascular diameter are rather stable for at least the duration of the experiments.

Detection of the platelets could be achieved by labelling them selectively with the fluorescent dye acridine red (Tangelder et al, 1982a). After injection of the dye into the marginal ear vein of the rabbit, flowing platelets could be observed with sufficient intensity during 2-8 minutes. With this procedure nearly all circulating platelets are labelled without activation of the platelets by the dye or induction of gross ultrastructural changes in the platelets (Tangelder et al, 1982a). The dose of acridine red used in these studies (about $6 \mathrm{mg} / \mathrm{kg}$ bodyweight), did not influence hematocrit or systemic platelet count.

An important finding of this thesis is that there is a difference in platelet distribution between arterioles and venules (chapter 4). In the vessel center, the relative platelet concentration is significantly lower in arterioles than in venules, whereas near the wall the opposite is observed. It is unlikely that the observed difference in platelet distribution between arterioles and venules is caused by a difference in shape. Almost circular venules and arterioles can be found in fixed mesentery (chapter 4, Fox et al, 1980). Mathematical calculations show that flattening of a circular vessel by $30 \%$, barely changes the platelet distribution profile in both arterioles and venules. The difference between both distributions becomes even more accentuated.

The platelet distribution, as found in venules in vivo, shows no correspondence with that obtained in vitro. Experiments performed with blood suspensions flowing in glass channels (Eckstein et all, 1988; Tilles and Eckstein, 1987) or slits (Palmer, 1967), showed that the platelet concentration near the wall was found to be 3 to 5 times higher than that in the 
center of the channel. These in vitro distributions are in agreement with those found in arterioles in both the study presented in this thesis and in a previous one (Tangelder et al, 1985). It should be noticed that in contrast to experiments performed in vitro, where the reaction of one cell type can be tested to changes in one variable, in vivo a great number of variables may vary simultaneously and determine the observed platelet distribution. The interaction between platelets, other blood cells and the vessel wall is a complex phenomenon, which may be influenced by several factors. Therefore, one should be careful in extrapolating results obtained in vitro to the in vivo situation.

To interpret blood platelet-vessel wall interactions in microvessels in a correct way, one has to be informed of wall shear rate and wall shear stress values. To be able to reliably assess these values, detailed description of the shape of the velocity profile is required. It has been shown that in arterioles the velocity profile in the vessel center is a flattened parabola (Tangelder et al, 1986). In venules, however, the shape of the profile in the core-fluid of straight vessel segments is not different from a parabola. In both venules and arterioles, the velocities near the wall are higher than expected in case of a parabolic profile (chapter 6). The finding that in venules the shape of the profiles in the core-fluid does not differ from a parabola, suggests that in these vessels the profile had reached its final shape after a distance of at least 5 vessel diameters, which is the distance of the site of observation, away from branch points. The fact that the observed velocity profiles are rather symmetric also indicates that disturbances of the profile due to side branches have generally disappeared within this distance. It is unlikely that differences in platelet distribution are responsible for the central blunting as observed in arterioles but not in venules, because the total platelet volume relative to total blood volume is less than $0.25 \%$. As pointed out in chapter 6 , the observed differences in velocity profile between arterioles and venules are probably not due to differences in centerline velocity or pulsatile flow. Because both vessel types differ in flow at branch points, it cannot be excluded that the confluent flow in venules contributes differently to the shape of the velocity profile as compared with the diverging flow at arteriolar branch points.

The flowing of platelets in arterioles at higher concentrations near the wall than in the center, does not result in a difference between mean platelet velocity and mean whole blood velocity; no tube Fåhraeus effect is present for platelets. In venules, the presence of a relative concentration peak more distant from the wall than in arterioles, in combination with a relatively high concentration in the center, results in an increased platelet 
velocity as compared to mean whole blood velocity. Hence, in venules a Fåhraeus effect for platelets is present and will result in a reduced local platelet concentration (chapter 7).

Based on the calculated Fahraeus effect, it seems that no significant changes occur in local platelet concentration in arterioles in rabbit mesentery. However, besides the tube Fahraeus effect, based on the velocity profile and the concentration distribution, the separation of phases at bifurcations, leading to a network Fåhraeus effect (Pries et al, 1986), should also be taken into account. Tangelder (1982) demonstrated that in arterioles, where the platelets flow preferentially near the wall, directly past a bifurcation the relative concentration peak on the side of the bifurcation has disappeared. A disproportional number of platelets has entered the side branch. Due to this skimming effect of platelets in arterioles, a nonhomogeneous distribution of platelets within a network will result ( $\Theta$ fjord and Clausen, 1986).

In arterioles, the shape of the velocity profile of FITC-labelled red blood cells (Tangelder et al, 1986) is similar to that of blood platelets. However, most of the red blood cells are travelling at streamlines with higher velocities, i.e. more towards the center of the vessel. This tendency of red blood cells to occupy streamlines with higher velocity probably explains the relatively low blood platelet concentration in the center of arterioles (Palmer, 1967; Turitto and Baumgartner 1975). At this moment it is not known whether the relatively high platelet concentration in the center of venules can be explained by a relative absence of red blood cells in this part of these microvessels, because to our best knowledge hematocrit distributions over the cross-sectional area of the microvessels have not been measured.

The tendency of red blood cells to form aggregates (Chien, 1980) might also play a role in the above mentioned difference in platelet distribution between arterioles and venules (Palmer, 1967), provided that differences in red blood cell aggregate formation exist between arterioles and venules. In chapter 4 and 5 , we therefore performed experiments on platelet distribution in arterioles and venules with several dextrans, which were injected intravenously in the rabbits. Neutral high molecular weight dextran (Dx500; mol wt:485.000), as well as negatively charged dextran (Dx500S; $17 \%$ sulfate groups), increased the platelet concentration in the center of arterioles and decreased it near the wall when compared to control arterioles. A similar but less pronounced effect was observed in venules. Injection of a neutral low molecular weight dextran (Dx40; mol wt:39.000), however, had little or no effect in arterioles, but decreased the 
platelet concentration in the center of venules and increased it near the wall. At the dose used ( $30 \mathrm{mg} / \mathrm{kg}$ bodyweight), no effect on red blood cell aggregation tendency was observed after injection of the low molecular weight dextran, while injection of the high molecular weight dextrans increased this variable approximately twofold (chapter 5). This leaves the notion of a possible contribution of red blood cell aggregation to the platelet concentration distributions intact. Although high molecular weight dextrans may induce platelet aggregation, partially by acting as macromolecular bridges between cells (Bygdeman and Eliasson, 1967), in none of our experiments platelet doublets, triplets or larger aggregates were observed.

A possible explanation for the observed increase in platelet concentration in the center of arterioles and venules after injection of high molecular dextran may be that in vivo an increased number of macromolecular bridges between red blood cells hampered their capability to disperse platelets from the vessel center. Another explanation might be that the high molecular weight dextrans act as bridging molecules between platelets and red blood cells. By such a mechanism red blood cells might trap platelets in the center. However, the validity of these explanations remains subject to further investigation.

Beside directly, via an interaction with platelets, dextrans may influence platelet distribution indirectly by changing red blood cell behavior. Red blood cell deformability and plasma viscosity both influence the tendency of red blood cells to migrate towards the vessel center (Goldsmith, 1972; Palmer and Jedrezejczyk, 1975). These two parameters are not influenced by the injected dose of the high molecular weight dextran (chapter 5). In addition, the low dose used does not change systemic hematocrit, mean arterial pressure or microvascular red blood cell velocity (chapter 4 and 5). Although dextran injection does not change microvascular flow velocity, it cannot be excluded that the shape of the velocity profile changes under influence of these dextrans. In case of a change in shape due to dextran, one might expect a higher degree of blunting (Rosenblum, 1972), leading to an increase in wall shear rate. It seems unlikely, however, that such an increase in wall shear rate can be held responsible for the increase in platelet concentration in the center of arterioles following dextran injection, because findings in vitro indicate that platelet distribution is independent of wall shear rate at values higher than $430 \mathrm{~s}^{-1}$. Generally, higher wall shear rates are found in arterioles in vivo (Tangelder et al, 1988).

Red bllood cell deformability is known to be influenced by oxygen pressure. (Lacelle and Weed, 1970). In rabbits, red blood cells are signifi- 
cantly less deformable during hypoxia, i.e. around $50 \mathrm{mmHg} \mathrm{pO}_{2}$, as compared to normoxic conditions (Hakim and Macek, 1988). In the feeding arteries of normally ventilated rabbits the $\mathrm{pO}_{2}$ is almost equal to the systemic arterial values $(90-100 \mathrm{mmHg})$, in contrast to venules where $\mathrm{pO}_{2}$ values below $50 \mathrm{mmHg} \mathrm{pO}_{2}$ can be found (oude Egbrink, 1989). These low $\mathrm{pO}_{2}$ values in venules may lead to less deformable red blood cells. Experiments performed in a Baumgartner perfusion chamber showed that less deformable human red blood cells can enhance platelet adhesion to subendothelium (Aarts et al, 1984). Based on these results one could conclude that more platelets will possibly be expelled to the vessel wall in venules than in arterioles. However, the opposite is found. Therefore, red blood cell deformability is probably not responsible for the observed difference in platelet distribution between arterioles and venules. An explanation for these contrasting findings is that rabbit red blood cells are smaller (average diameter: $6.9 \mu \mathrm{m}$ ) than human red blood cells (average diameter: $7.5 \mu \mathrm{m}$ ), which in case of the rabbit red blood cells may lead to a much smaller effect as far as expelling of platelets to the vessel wall is concerned (Aarts et al, 1983).

In venules, but not in arterioles, there seems to be a relative exclusion for platelets close to the vessel wall (chapter 4 and 6). This difference can at best only partially be explained by differences in platelet orientation between arterioles and venules, if any, in this region. This points to the existence of an almost platelet free layer near the wall, in contrast to arterioles in which the flowing platelets can be observed in apparent contact with the vessel wall (Tangelder et al, 1986). Since in arterioles platelets do come as close to the vessel wall as $0.5 \mu \mathrm{m}$, wall shear rate could be estimated rather accurately in these microvessels. In venules, however, the estimation will be less accurate, because larger extrapolations to zero flow at the wall (no-slip condition) are required due to the relative exclusion of platelets near the venular wall.

This difference in wall exclusion is also not caused by the presence of rolling and sticking leukocytes, a phenomenon which in the exteriorized mesentery is observed only in venules. Significant reduction of leukocyte rolling in venules by injecting high molecular weight dextran sulfate had no effect on platelet distribution (chapter 4). It is also unlikely that the observed difference in wall exclusion was caused by activation of the blood platelets due to the acridine red used to label the platelets fluorescently. Experiments performed by oude Egbrink and co-workers (1992) revealed that rolling of leukocytes is attenuated by activated platelets, either directly or through an effect on endothelial cells. In our experiments injection of 
acridine red in the rabbit did not induce a change in the level of leukocyte rolling, which indicates that activation of platelets does not occur. This is in agreement with in vitro experiments, which showed that acridine red does not induced platelet aggregation (Tangelder et al, 1982a).

The observed wall exclusion of platelets in venules and not in arterioles can most likely be ascribed to differences in wall structure and/or function between both vessel types. Because other findings also suggest a difference in endothelial structure between these microvessels, e.g. differences in permeability (De Clerck et al, 1985), in their thromboembolic reaction to microinjury (oude Egbrink et al, 1988) and the margination of leukocytes (Tangelder and Arfors, 1991), further research should therefore include the investigation of the glycocalyx composition and electrical charge of arteriolar and venular endothelium.

In conclusion, the observed differences in platelet distribution and velocity profile between arterioles and venules are probably not caused by leukocyte rolling. Neither differences in red blood cell velocity nor in vessel shape can explain the observed difference. To which extent local hematocrit concentration over the cross-sectional area and red blood cell aggregation play a role is subject to further investigation. Another consistent finding is that a relatively large wall exclusion of platelets is present in venules and not in arterioles, suggesting differences in endothelial surface characteristics between both vessel types. 


\section{REFERENCES}

Aarts PAMM, Bolhuis PA, Sakariassen KS, Heethaar RM and Sixma JJ. Red blood cell size is important for adherence of blood platelets to artery subendothelium. Blood 62:214-220, 1983.

Aarts PAMM, Heethaar RM and Sixma JJ. Red blood cell deformability influences platelet-vessel wall interaction in flowing blood. Blood 64:1228-1233, 1984.

Adelson E, Crosby WH and Roeder WH. Further studies of a hemostatic defect caused by intravenous dextran. J Lab Clin Med 45:441-448, 1955.

Albrecht KH, Gaehtgens P, Pries A and Heuser M. The Fåhraeus effect in narrow capillaries. Microvasc Res 18:33-47, 1979

Arfors KE and Bergqvist D. Microvascular haemostatic plug formation in the rabbit mesentery. Bibltheca Haemat 41:84-97, 1975.

Atherton A and Born GVR. Quantitative investigations of the adhesiveness of circulating polymorphonuclear leukocytes to blood vessel walls. I Physiol 222:447-474, 1972.

Badimon L, Badimon JJ, Turitto VT and Fuster V. Thrombosis: studies under flow conditions. Ann NY Acad Sci 516:527-540, 1987.

Bagge U, Brånemark PI, Karlsson K and Skalak R. Three-dimensional observations of red blood cell deformation in capillaries. Blood Cells 6:231, 1980.

Baker $\mathrm{M}$ and Wayland $\mathrm{H}$. On-line volume flow rate and velocity profile measurement for blood in microvessels. Microvasc Res 7:131-143, 1974.

Bergqvist D. Haemostatic plug formation in the rabbit mesentery. Upsala J Med Sci 79:28-38, 1974.

Bergqvist D and Arfors KE. Haemostasis in the microvasculature of the rabbit mesentery, effects of some pharmacological agents of current interest in haemostasis and thrombosis. Haemostasis 5:74-84, 1976.

Bizzozero J. Ueber einen neuen Formbestandtheil des Blutes und dessen Rolle bei der Thrombose und der Blutgerinnung. Virchows Arch Pathol Anat Physiol Klin Med 90:261-332, 1882.

Bloch EH. A quantitative study of the hemodynamics in the living microvascular system. Am J Anat 110:125-153, 1962.

Bloch EH. High speed cinephotography of the microvascular system. Hemorheology: proceedings of the first international conference. Copley AL (ed.), Pergamon Press, New York, pp 655-667, 1968.

Braasch D.Red cell deformability and capillary blood flow. Physiol Reviews 51(4):679701, 1971.

Bradley JV. Distribution free statistical tests. Prentice Hall, Inc., Englewood Cliffs, New York, 1968. 
Bryant MF, Bloom WL and Brewer SS. Study of the anti-thrombotic properties of dextrans of large molecular weight. J Cardiovasc Surg 5:48-52, 1964.

Bromwell AW, Artz CP and Sako Y. Evaluation of blood loss from standardized wound after dextran. Surg Forum 5:809, 1954.

Brooks DE. Effects of neutral polymers on the surface potentials of cells and other charged particles. III. Experimental studies on the dextran/erythrocyte system. J Colloid Interface Sci 43:700-713, 1973.

Bygdeman S and Eliasson R. Effect of dextrans on platelet adhesiveness and aggregation. Scan J Clin Lab 20: 17-23, 1967.

Bugliarello G and Hayden JW. Detailled characteristics of flow of blood in vitro. Trans Soc Rheology 7:209-230, 1963

Bugliarello $G$ and Sevilla $J$. Velocity distribution and other characteristics of steady and pulsatile blood flow in fine glass tubes. Biorheology 7:85-107, 1970

Caro CG, Pedley TJ, Schroter RC and Seed WA. The mechanics of the circulation, Oxford University Press, Oxford, 1978.

Chaffey CE, Brenner $\mathrm{H}$ and Mason SG. Particle motions in sheared suspension. XVIII: Wall migration (theoretical). Rheol Acta 4:64-72, 1965.

Chien S, Usami S and Bertles JF. Abnormal Rheology of oxygenated blood in Sickle Cell Anemia. J Clin Invest 49:623-634, 1970a.

Chien S, Usami S, Dellenback RJ and Gregersen MI. Shear dependent deformation of erythrocytes in theology of human blood. Am J Physiol 219:136-142, $1970 \mathrm{~b}$.

Chien S, Usami S, Dellenback RJ and Bryant CA. Comparative hemorheology he matological implications of species differences in blood viscosity. Biorheology 8: 35-57, 1971.

Chien, S. and K-M. Jan. Ultrastructural basis of the mechanism of rouleaux formation. Microvasc Res 5: 155-166, 1973.

Chien S, Simchon S, Abott RE and Jan KM. Surface absorption of dextrans on human red cell membrane. J Colloid Interface Sci 62:461, 1977.

Chien S. Aggregation of red blood cells: An electrochemical and colloid chemical problem. Adv Chem Ser 188: 1-38, 1980.

Chien S, Sung LA, Simchon S, Lee MML, Jan KM and Skalak R. Energy balance in red cell interactions. Ann NY Acad Sci 416:190-207, 1983

Chien S, Usami S and Skalak R. Blood flow in small tubes. In; Handbook of Physiology: section 2: The Cardiovascular System, vol IV: Microcirculation, part 2. Renkin EM and Michel C (eds), Am Physiol Soc, Bethesda, Maryland, pp 217-249, 1984.

Chien S. White cell rheology. In: Clinical blood rheology. Lowe GDO (ed), CRC Press, Boca Raton, pp 87-109, 1988.

Copley AF and Seaman GVF. The meaning of the terms rheology, biorheology and hemorheology. Clin Hemorheol 1:117, 1981.

Daniel WW. Biostatistics: a foundation for analysis in the health sciences. John Wiley and Sons (eds), New York, 1983.

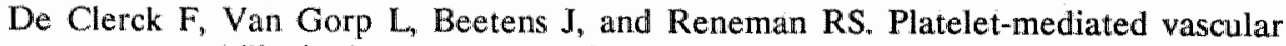
permeability in the rat: a predominant role for 5-hydroxytriptamine. Thromb Res $38: 321-339,1985$.

Desjardins C and Duling BR. Heparinase treatment suggest a role for the endothelial cell glycocalyx in regulation of capillary hematocrit. Am J Physiol 258:H647-H654, 
1990.

Driessen GK, Heidmann $\mathbf{H}$ and Schmid-Schönbein $H$. Effect of haematocrit on red blood cell velocity in the capillaries of the rat mesentery during hemodilution and hemoconcentration. Pflügers Arch 380:1-6, 1979.

Engeset J, Stalker AL and Matheson NA. Effects of dextran 40 on red blood cell ag gregation in rabbits. Cardiovasc Res 1:379-384, 1967.

Escolar G, Garrido M, Aznar-Salatti J, Ordinas A and Bastida E. Comparison between human umbilical artery and rabbit aorta as substrata for platelet adhesion and platelet thrombus formation under flow conditions. Blood vessels 28:520-531, 1991.

Eckstein EC, Tilles AW and Millero FJ. Conditions for the occurrence of large near-wall excesses of small particles during blood flow. Microvasc Res 36:31-39, 1988.

Făhraeus R. Die Stromungsverhaltnisse und die Verteilung der Blutzellen im Gefasssystem. Klin Wschr 7:100-106, 1928

Fåhraeus R. The suspension stability of blood. Physiol Rev 9:241-274, 1929.

Fahraeus $\mathbf{R}$ and Lindquist $T$. The viscosity of blood in narrow capillary tubes. Am $J$ Physiol 96:562-568, 1931.

Fox J, Galey F and Wayland $\mathbf{H}$. Action of histamine on the mesenteric microvasculature. Microvasc Res 19:108-126, 1980.

Gaehtgens P, Albrecht KH and Kreutz. F. Fåhraeus effect and cell screening during tube flow of human blood. I. Effect of variation of flow rate. Biorheology 15:147-154, 1978

Gaehtgens P. Flow of blood through narrow capillaries: rheological mechanisms deter mining capillary hematocrit and apparent viscosity. Biorheology $17: 183-189,1980$

Gelin LE and Ingelman B. Rheomacrodex - a new dextran solution for rheological treatment of impaired capillary flow. Acta Chir Scand 122:294-302, 1961.

Goldsmith HL and Mason SG. The microrheology of dispersions. In: Rheology: theory and applications, vol IV. Eirich FR (ed), Academic Press, New York, pp 85-250, 1967.

Goldsmith HL. Microscopic flow properties of red blood cells. Fed Proc 26:1813-1820, 1967.

Goldsmith HL. Red cell motions and wall interactions in tube flow. Fed Proc 30:1578$1588,1971$.

Goldsmith HL. The flow of model particles and blood cells and its relation to throm bogenesis. In: Progress in hemostasis and thrombosis, vol.1. Spaet TH (ed), Grune and Stratton, New York, pp 97-139, 1972.

Goldsmith HL. The microrheology of human erythrocyte suspensions. Applied mecha nics: proceedings of the thirteenth international congress of theoretical and applied mechanics. Beeker $\mathrm{E}$ and Mikhailov GK (eds), Springer, Berlin-New York, pp 85-103, 1973.

Goldsmith HL and Marlow JC. Flow behavior of erythrocytes II, Particle motions in concentrated suspensions of ghost cells. J Colloid Int Scil 71:383 407, 1979.

Goldsmith HL and Spain S. Margination of leukocytes in blood flow through small tubes. Microvasc Res 27:204-222, 1984.

Goldsmith HL and Turitto VT. Rheological aspects of thrombosis and haemostasis: basic principles and applications. Thromb Haemostas 55:415-435, 1986. 
Gruber UF. Blood Replacement, Springer, Berlin, 1969.

Hakim TS and Macek AS. Effect of hypoxia on erythrocyte deformability in different species. Biorheology 25:857-868, 1988.

Hardeman MR, Bauersachs RM and Meiselman HJ. RBC laser diffractomery and RBC aggregometry with a rotational viscometer: comparison with rheoscope and myrenne aggregometer. Clinical Hemorheology 8:581-593, 1988.

Hardeman MR and Rie MA. Impaired Erythrocyte Deformability in Psoriasis patients treated with cyclosporin. Thromb Haemostas 65 (6):984, 1991.

Heltianu C, Simionescu M and Simionescu N. Histamine receptors of the microvascular endothelium revealed in situ with a histamine-ferritin conjugate: characteristic high-affinity binding sites in venules. J Cell Biol 93:357-364, 1982.

Howard JM, Teng CT and Loeffler RK. Studies of dextrans of various molecular sizes. Ann Surg 143:369, 1956.

House SD and Lipowsky HH. Leukocyte-endothelium adhesion: microdynamics in the mesentery of the cat. Microvas Res 34:363-379, 1987.

Hultman E. Carbohydrates and related compounds. A: Glucose, galactose, fructose, inulin and ketone bodies in blood and urine In: Clinical Biochemistry, Principles and Methods. Curtius HCh and Roth M (eds), De Gruyter, Berlin, Germany, pp 908-930, 1974.

Intaglietta $\mathrm{M}$ and Tompkins WR. Microvascular measurements by video image shearing and splitting. Microvasc Res 5: 309-312, 1973.

Jacobeaus $U$. Studies on the effect of dextran on the coagulation of blood. Acta med scandinav 157: supplementum 322, 1957.

James J. Light Microscopic Techniques In Biology and Medicine. Martinus Nijhoff, The Hague, 1976.

Jan KM. The disaggregating affect of dextran 40 on red cell aggregation in macromolecular suspensions. Biorheology 19:543-554, 1982.

Jan KM and Chien S. Role of surface charge in red blood cell interactions. J Gen Physiol 61:638-654, 1973.

Jeffery GB. The motion of ellipsoidal particles immersed in a viscous fluid. Proc R Soc Lond a102, 161-179, 1922.

Kanzow G, Pries AR and Gaehtgens P. Analysis of the hematocrit distribution in the mesenteric microcirculation. Int J Microcirc Clin Exp 1:67-79, 1982.

Karino T and Goldsmith HL. Haemostasis and Thrombosis: rheological factors in thrombosis and hemostasis. Bloom AL (ed), pp 739-742, 1987.

Karnis A and Mason SG. Particle motions in sheared suspensions. XXIII. Wall migration of fluid drops. J Colloid Interface Sci 24:164-169, 1967.

Kiesewetter H, Schmid-Schönbein H, Radtke H and Stolwerg G. In vitro demonstration of collateral blood viscidation. Flow measurements in a model of vascular networks. Microvase Res 17:12-19, 1979.

Kovacs IB, Tigyi-Sebes A, Trombitas K and Gorog P. Evans Blue: An ideal energyabsorbing material to produce intravascular microinjury by HE-NE gas laser. Microvasc Res 10:107-124, 1975.

Kozma C. The anatomy, physiology, and biochemistry of the rabbit. In: The biology of the laboratory rabbit. Weisbroth SE, Flatt RE and Kraus AL (eds). Academic Press, New York, pp 50-72, 1974. 
Kurland GS. The marginal plasma layer. In: Blood flow and microcirculation. John Wiley and sons (eds), New York, pp 72-87, 1974.

Kwaan HC, Harding F and Astrup T. Platelet behavior in small blood vessels in vivo. Thromb Diath Haemorrh 26:207-220, 1967.

Lacelle PI and Weed RI. Low oxygen pressure: a cause of erythrocyte membrane rigidity. $J$ Clin Invest 49:54a, 1970.

Langdell RD, Adelson E, Furth FW and Crosby WH. Dextran and prolonged bleeding time. JAMA 166:346, 1958.

Lillie RD. H.J. Conn's Biological stains. Baltimore: The Williams and Wilkins Company, 1977.

Lipowsky HH, Kovalcheck S and Zweifach BW. The distribution of blood rheological parameters in the microvasculature of the cat mesentery, Circ Res 43:738-749, 1978

Lipowsky HH, Usami S and Chien S. In vivo measurements of "apparent viscosity" and microvessel hematocrit in the mesentery of the cat. Microvasc Res 19:297-319, 1980.

Lowe GDO. Rheology of paraproteinemias and leukemias. In: Clinical blood rheology. Lowe GDO (ed) CRC Press, Boca Raton, pp 67-87, 1988.

Maupin B. Blood platelets in man and animals. Pergamon Press, New York, 1969.

Mayer GA, Fridrich J, Newell J and Szivek J. Plasma components and blood viscosity. Biorheology 3:171-176, 1966.

Meiselman HJ, Merril EW, Salzman EW, Gilliland ER and Pelletier GA. Effect of dextran on rheology of human blood: low shear viscometry. J Appl Physiol 22:480486, 1967.

Merril EW. Rheology of blood. Physiol Rev 49:863-888, 1969.

Murphy JR. Erythrocyte shape and blood viscosity. Proceedings of the first International Conference of Hemorheology, Reykjavik, Iceland, pp 469-477, 1966.

Nakajima K, Shirai K, Aoki $M$ and Yasunaga $K$. Effects of dextran sulfate on platelet aggregation in rabbits. Acta Haem Jap 41:803-809, 1978.

Nash $\mathrm{GB}$ and Meiselman $\mathrm{HJ}$. Effects of dextran and polyvinylpyrrolidone on red cell geometry and membrane elasticity. Ann NY Acad Sci 416:255-262, 1983.

Nobis U, Pries AR, Cokelet GR and Gaehtgens P. Radial distribution of white cells during blood flow in small tubes. Microvasc Res 29:295-304, 1985.

Ofjord ES and Clausen G. Relative flow of blood cells, platelets and microspheres in outer and inner renal cortex. Am J Physiol 251:H242-H246, 1986.

oude Egbrink MGA, Tangelder GJ, Slaaf DW and Reneman RS.Thromboembolic reaction following wall puncture in arterioles and venules of the rabbit mesentery. Thromb Haemost 59:23-28, 1988.

oude Egbrink MGA, Tangelder GJ, Slaaf DW and Reneman RS. Effect of blood gases and $\mathrm{pH}$ on thromboembolic reactions in rabbit mesenteric microvessels. Pflügers Arch 414:324-330, 1989.

oude Egbrink MGA, Tangelder GJ, Slaaf DW and Reneman RS. Fluid dynamics and the thromboembolic reaction in mesenteric arterioles and venules. Am $\mathbf{J}$ Physiol 260:H1826-1833, 1991a.

oude Egbrink MGA, Tangelder GJ, Slaaf DW and Reneman RS. Wall shear rate and blood cell vessel wall interactions in microvessels. Rev Port Hemorreol 5 (supl. 


\section{2):1339-139, $1991 \mathrm{~b}$.}

oude Egbrink MGA, Tangelder GJ, Slaaf DW and Reneman RS. Influence of plateletvessel wall interactions on leukocyte rolling in vivo. Circ Res 70:355-363, 1992.

Palmer AA. Axial drift of cells and partial plasma skimming in blood flowing through glass slits. Am J Physiol 209:1115-1122, 1965.

Palmer AA. Platelet and leukocyte skimming. Bibl anat: vol 9, pp. 300-303, 1967.

Palmer AA and Jedrzejczyk HJ. The influence of rouleaux on the resistance to flow through capillary channels at various shear rates. Biorheology 12:265-270, 1975.

Palmer AA and WH Betts. The axial drift of fresh and acetaldehyde-thardened erythrocytes in $25 \mu \mathrm{m}$ capillary slits of various lengths. Biorheology 12:283-292, 1975.

Peeters ME, Gil D, Teske E, Eyzenbach V, Brom vd WE, Lumeij JT and de Vries HW. Four methods for general anaesthesia in the rabbit: a comparative stu. Laboratory Animals 22:355-360, 1988.

Peeze-Binkhorst FM, Kuipers H, Heymans J, Frederik PM, Slaaf DW, Tangelder GJ and Reneman RS. Exercise-induced focal skeletal muscle fiber degeneration and capillary morphology. J Appl Physiol 66:2857-2865, 1989.

Pfafferott C, Nash GB and Meiselman HJ. Red blood cell deformation in shear flow: Effects of internal and external phase viscosity and in vivo aging. Biophysical Journal 47: 695-704, 1985.

Poole JCF and French JE. Thrombosis. J Atheroscler Res 1:251-282, 1961.

Popilskis SJ, Oz MC, Gorman P, Florestal A and Kohn DF. Comparison of Xylazine with Tiletamine-Zolazepam (Telazol) and Xylazine-ketamine anesthesia in rabbits. Lab Anim Sci 41:51-53, 1991.

Pries AR, Ley K and Gaehtgens P. Generalization of the Fahraeus principle for microvessel networks. Am J Physio! 251: H1324-H1332, 1986

Reneman RS, Woldhuis B, oude Egbrink MGA, Slaaf DW and Tangelder GJ. Concentration and velocity profiles of blood cells in the microcirculation. In: Frontiers of cardiovascular Engineering. NHC Hwang, VT Turitto and MRT Yen (eds), Plenum, New York 1993, pp 25-40.

Replogle RL, Meiselman HJ and Merril EW. Clinical implications of blood rheological studies. Circulation 36:148-160, 1967.

Rhodin JAG. The ultrastructure of mammalian arterioles and precapillary Sphincters. J. Ultrastructure Research 18:181-223, 1967.

Rhodin JAG. Ultrastructure of mammalian venous capillaries, venules and small collecting veins. J. Ultrastructure Research 25:452-500, 1968.

Rosenblum WI. Ratio of red cell velocities near the vessel wall to velocities at the vessel center in cerebral microcirculation, and apparent effect of blood viscosity on this ratio. Microvasc Res 4:98-101, 1972.

Rosenblum WI. Red cell velocity and plasma transit time in the cerebral microcirculation of spherocytic deer mice. Circ Res 39:452-454, 1976.

Sarelius IH and Duling BR. Direct measurement of microvessel hematocrit, red cell flux, velocity and transit time. Am J Physiol 243:H1018-H1026, 1982.

Schalm OW, Jain NC and Carroll EJ. Veterinary Hematology, Chapter 4: Normal values in blood of laboratory, Fur-bearing and Miscellaneous Zoo and Wild Animals. Lea and Febiger (eds), Philadelphia, USA, pp 219-228, 1975. 
Schmid-Schönbein $\mathbf{H}$ and Wells R. Fluid drop-like transition of erythrocytes under shear. Science $165: 288,1969$.

Schmid-Schönbein GW and Zweifach BW. RBC velocity profiles in arterioles and venules in the rabbit omentum Microvasc Res 10:153-164, 1975.

Schmid-Schönbein H, Usami S, Skalak R and Chien S. Cell distributions in capillary networks. Microvase Res 19:18-44, 1980a.

Schmid-Schönbein H, Usami S, Skalak R and Chien S. The interactions of leukocytes and erythrocytes in capillary and postcapillary vessels. Microvasc Res 19:45-70, $1980 \mathrm{~b}$.

Segre G and Silberberg A. Behaviour of macroscopic rigid spheres in Poiseuille flow. Part 2: Experimental results and interpretation. J Fluid Mech 14:136-157, 1962.

Siegel S. Nonparametric statistics for the behavioral sciences. McGraw-Hill Kogakusha, LTD, Tokyo, 1956.

Silver MD and Stehbens WE. The behavior of blood platelets in vivo. Quart J Exp Physiol 50:241-246, 1965. S

Simchon S, Jan KM and Chien S. Influence of reduced red cell deformability on regional blood flow. Am J Physiol 253:H898-H903, 1987.

Sixma JJ and Wester J. The haemostatic plug. Semin Haematol 14:265-299, 1977.

Slaaf DW, Rood JPSM, Tangelder GJ, Jeurens TJM, Alewijnse R, Reneman RS and Arts T. A bidirectional optical (BDO) three-stage prism grating system for on-line measurement of red blood cell velocity in microvessels. Microvasc Res 22:110-122, 1981.

Slaaf DW, Alewijnse R and Wayland $\mathrm{H}$. Use of telescopic imaging in intravital micros copy: a simple solution for conventional microscopes. Int J Microcirc Clin Exp $1: 121-134,1982$.

Slaaf DW, Arts T, Jeurens TJM, Tangelder GJ and Reneman RS. Electronic measure ments of red blood cell velocity and volume flow in microvessels. In: Investigative microtechniques in medicine and biology. Chayen $J$ and Bitensky $L$ (eds). Marcel Dekker Inc, New York, 327-364, 1984.

Slaaf DW, Tangelder GJ and Reneman RS. Physics of the microcirculation. In press.

Stoltz, JF. Electrochemical properties of platelets: clinical and pharmacological ap plications. Ann NY Acad Sci 416, 1983.

Tangelder GJ.Distribution and orientation of blood platelets flowing in small arterioles. PhD-thesis, University of Limburg, Maastricht, The Netherlands, 1982.

Tangelder GJ, Slaaf DW and Reneman RS. Fluorescent labelling of blood platelets in vivo. Thromb Res 28:803-820, 1982a.

Tangelder GJ, Slaaf DW, Teirlinck HC, Alewijnse R and Reneman RS. Localization within a thin optical section of fluorescent blood platelets flowing in a microvessel. Microvasc Res 23:214-230, $1982 \mathrm{~b}$.

Tangelder GJ, Slaaf DW and Reneman RS. Skeletal muscle microcirculation and changes in transmural and perfusion pressure. Prog Appl Microcirc vol. 5, pp 93$108,1984$.

Tangelder GJ, Teirlinck HC, Slaaf DW and Reneman RS. Distribution of blood platelets flowing in arterioles. Am J Physiol 248:H318-H323, 1985.

Tangelder GJ, Slaaf DW, Muijtjens AMM, Arts T, Oude Egbrink MGA and Reneman RS. Velocity profiles of blood platelets and red blood cells flowing in arterioles 
of the rabbit mesentery. Circ Res 59:505-514, 1986 .

Tangelder GJ, Slaaf DW and Reneman RS. In vivo behavior of fluorescently labelled blood cells. Microcirculation: An Update. Proceedings of the Fourth World Congress for Microcirculation, Tokyo, Japan, pp. 314-317, 1987.

Tangelder GJ, Slaaf DW, Arts T and Reneman RS. Wall shear rate in arterioles in vivo: least estimate from platelet velocity profiles. Am J Physiol 254:H1059-1064, 1988. Tangelder GJ, oude Egbrink MGA, Slaaf DW and Reneman RS. Blood platelets: an overview. J Reconstr Mïcrosurg 5:167-171, 1989.

Tangelder GJ and Arfors KE. Inhibition of leukocyte rolling in venules by protamine and sulfated polysaccharides. Blood 77:1565-1571, 1991.

Teirlinck HC, Tangelder GJ, Slaaf DW, Muijtjens AMM, Arts T and Reneman RS. Orientation and diameter distributions of rabbit blood platelets flowing in small arterioles. Biorheology 21:317-331, 1984.

Thornson $G$ and Hint $H$. Aggregation, sedimentation and intravascular sludging of erythrocytes. Acta Chir Scand Suppl 154;1-50, 1950.

Tilles AW and Eckstein EC. The near-wall excess of platelet-sized particles in blood flow: Its dependence on hematocrit and wall shear rate. Microvasc Res 33:211223, 1987.

Tocantins LM. Historical notes on blood platelets. Blood 3:1073-1082, 1948.

Turitto VT. Blood viscosity, mass transport and thrombogenesis, in: Progress in Hemostasis and Thrombosis 6, Speat TH (ed). Grune and Stratton, New York, pp 139$177,1982$.

Turitto VT and Baumgartner HR. Platelet interaction with subendothelium in a perfusion system: Physical role of red blood cells. Microvasc Res 9:335-344, 1975.

Turitto VT and Baumgartner HR. Platelet interaction with subendothelium in flowing rabbit blood:Effect of blood shear-rate. Microvasc Res 17:38-54, 1979.

Turitto VT and Weiss HJ. Red blood cells: Their dual role in thrombus formation. Science 207:541-543, 1980.

Wells RE, Denton R and Merril EW. Measurement of viscosity of biologic fluids by cone plate viscometer. J Lab Clin Med 57:646-656, 1961.

Whitmore RL and Stalker AL. Red cell deformation in the microcirculation. Biorheology $13: 115-125,1976$.

Woldhuis B, Tangelder GJ, Slaaf DW and Reneman RS. Concentration profile of blood platelets differ in arterioles and venules. Am J Physiol 262:H1217-H1223, 1992.

Woldhuis B, Tangelder GJ, Slaaf DW and Reneman RS. Influence of dextrans on platelet distribution in arterioles and venules. Eur J Physiol (Pflügers Arch), in press.

Woldhuis B, Tangelder GJ, Slaaf DW and Reneman RS. Velocity profiles in rabbit mesenteric venules in vivo. Submitted.

Zilow EP and Linderkamp O. Viscosity reduction of red blood cells from preterm and full-term neonates and adult in narrow tubes (Fåhraeus-Lindqvist effect). Pediatr Res 25:595-597, 1989. 


\section{SUMMARY}

Blood platelets are the smallest cellular elements in the blood and they play a key role in the reaction of blood to vessel wall injury, i.e. in hemostasis and thrombosis. For these functions platelets have to come close by or in contact with the vessel wall. Experiments performed in vivo in arterioles and in vitro in small glass tubes show indeed a higher platelet concentration near the wall than in the center. By contrast, only a few studies exist on the rheology of blood in venules. It was therefore the aim of this thesis to study flow behavior of blood platelets in venules in vivo and compare this behavior with that in arterioles and with results obtained in vitro.

All experiments were performed in venules and arterioles of the rabbit mesentery. When using bright field microscopy, only platelets flowing near the vessel wall can be observed in these vessels, while detection of individual platelets located in the vessel center is hampered by the presence of the many red blood cells. However, after in vivo labelling with the fluorescent dye acridine red, visualization of all individual fast flowing platelets in these microvessels is possible using intravital fluorescence microscopy.

Segments of venules and arterioles ranging in diameter between 15 and $35 \mu \mathrm{m}$ were selected. Experiments were performed with position of sharp focus in the median plane of the vessels, i.e. the focal position yielding the widest vessel diameter. After injection with acridine red, flashes of short duration $(<0.1 \mathrm{~ms})$ were given to prevent a smeared image due to platelet movement. For the analysis of the data a transparent sheet was positioned over the monitor screen. Only platelets flowing within a thin optical section ( 5 to $7 \mu \mathrm{m}$ ) were used for analysis. According to the direction of flow, the left and right vessel walls were drawn. For each platelet marked, the distance between the centroid of the platelet (i.e. its center of gravity) and the vessel wall, as well as the vessel diameter at that specific point, were determined and the relative position of the platelet was calculated. For comparison between arterioles and venules relative frequency distributions were constructed.

The first experimental study (chapter 4) showed that the relative 
platelet concentration distribution differs greatly in venules and arterioles. Firstly, in the center of the vessel the platelet concentration is significantly higher in venules than in arterioles, while near the wall the opposite is observed. Secondly, close to the wall there seems to be a relative exclusion of platelets in venules. This exclusion could not be explained by the presence of marginating leukocytes in venules but not in arterioles, because intravenous injection of a sulfated dextran with high molecular weight, a compound known to inhibit leukocyte rolling, did not eliminate or reduce the relative platelet exclusion at the venular wall. Since it even tended to increase this wall exclusion of platelets, we also tested its effect and that of neutral dextrans, on platelet distribution in arterioles.

Dextrans are long chains of 1-6-and 1-4-linked glucose units, which bind to the surface of many cells, including platelets, red blood cells and endothelium. A well known effect of the binding of high molecular weight dextrans to red blood cells is an increase in their tendency to aggregate. Dextrans may also influence other rheological parameters, such as plasma viscosity and red blood cell deformability.

In chapter 5 we investigated whether a low dose $(30 \mathrm{mg} / \mathrm{kg} \mathrm{IV})$ of a low molecular weight dextran (Dx40), a neutral high molecular weight dextran (Dx500) or a high molecular weight dextran charged negatively with sulfate groups (Dx500S) influences platelet distribution. In arterioles, both high molecular weight dextrans increased the relative platelet concentration in the center and reduced it near the wall. In venules, a similar but less pronounced effect was observed. The low molecular weight dextran did not significantly change platelet distribution in arterioles, but in venules their concentration decreased in the center and increased at the wall. Dextran injection did not change microvascular flow velocity or plasma viscosity, suggesting that the observed changes in arteriolar platelet distribution were caused by binding of the dextran to the surface of platelets and/or red blood cells and/or endothelium.

To interpret blood platelet-vessel wall interactions in microvessels in a correct way, one has to be informed about wall shear rate and wall shear stress values. To be able to reliably assess these values, detailed description of the shape of the velocity profile is required. Chapter 6 deals with the velocity profile in venules. In venules the velocity profiles are rather symmetric and differ significantly from those in arterioles. Adequate description requires in both vessel types a two fluid-model: a thin layer of fluid at the wall with a significantly lower apparent viscosity than the fluid in the remaining core of the vessel. In venules, the shape of the profile in this core did not differ from a parabola, while in arterioles it was more blunt. 
As shown above, in microvessels the concentration of blood platelets and leukocytes is not uniform over the microvessel cross-section. This also holds for red blood cells. The latter, for example, preferentially flow in the areas with higher flow velocities, i.e. in the vessel center. This results in a mean red blood cell velocity that is higher than mean whole blood velocity. Consequently, the local red blood concentration in the vessel has to be lower than for homogeneously distributed red blood cells, because otherwise an accumulation of these cells would occur within the microcirculation. This is called the Fåhraeus effect. We investigated whether a similar effect also applies to blood platelets (chapter 7). Calculations demonstrate that in arterioles, despite a higher platelet concentration near the wall than in the center, the governing rheological conditions do not induce an inverted platelet Fåhraeus effect, as one would have expected. By contrast, in venules a higher mean velocity for platelets than for whole blood was found, which results in a platelet Fåhraeus effect and, hence, in a reduced mean local platelet concentration.

The two main findings obtained in this thesis, i.e. a difference in platelet distribution and in platelet vellocity profile between venules and arterioles in vivo, cannot be explained simply by hemodynamic differences e.g. flow velocity and pulsatility. These differences are also probably not caused by the presence of leukocyte margination in venules, but not in arterioles. Important factors to explain the observed differences between arterioles and venules might be the influence of red blood cells on the flow behavior of platelets and the position of a vessel within the vascular network, leading to diverging flow in arterioles and in converging flow in venules. The observed wall exclusion of platelets in venules and not in arterioles as described in chapters 4 and 6 can most likely be ascribed to differences in wall structure and/or function between both vessel types. 


\section{SAMENVATTING}

Het bloed is een vloeistof waarin zich naast eiwitten en andere stoffen drie verschillende typen bloedcellen bevinden. De rode bloedcellen, die ongeveer $40 \%$ van het volume innemen, zorgen voor transport van zuurstof en koolzuur. Witte bloedcellen hebben een belangrijke taak bij de afweer tegen infecties. Het bloedplaatje is het kleinste type bloedcel en noodzakelijk bij het stoppen van een bloeding na een beschadiging van een bloedvat.

De in het bloed stromende bloedplaatjes kunnen alleen hechten aan de binnenkant van een beschadigd bloedvat wanneer ze hiermee in aanraking komen. Er ontstaat een plaatjesprop, waaraan zich steeds meer plaatjes hechten zodat uiteindelijk de bloeding stopt. Het in contact komen van de bloedplaatjes met de wand en met elkaar hangt af van de manier waarop de bloedplaatjes zich bewegen in het stromend bloed, dat wil zeggen van hun stromingsgedrag (rheologie). Het onderzoek naar dit stromingsgedrag van bloedplaatjes kan zowel in glazen buisjes (in vitro) als in het levende organisme (in vivo) worden uitgevoerd.

Om alle cellen van het lichaam goed te laten functioneren is het noodzakelijk dat het bloed wordt vervoerd naar alle delen van het lichaam. Het bloed stroomt door een netwerk van buisjes, het zgn vaatstelsel. Dit vaatstelsel strekt zich tot in de kleinste delen van het lichaam uit. Het bloed dat zich in dit vaatstelsel bevindt, zorgt ervoor dat alle cellen worden voorzien van voldoende zuurstof en voedingsstoffen, terwijl koolzuur en afvalstoffen worden afgevoerd. Vanuit het hart wordt het bloed gepompt in grote vaten, die zich vertakken in steeds kleinere vaten (arteriolen) tot ze uiteindelijk uitmonden in haarvaten. In deze haarvaten vindt de uitwisseling plaats van stoffen tussen bloed en cellen. Het bloed wordt vanuit de haarvaten afgevoerd via de kleine vaten (venulen), die samenvloeien tot steeds grotere vaten en uiteindelijk uitkomen in het hart.

Het doel van het in dit proefschrift beschreven onderzoek was om in het levende dier (in vivo) het stromingsgedrag van bloedplaatjes in de kleine afvoerende vaten (venulen) te bestuderen. 


\section{Het onderzoek}

In het onderzoek werd gebruik gemaakt van venulen in het buikvlies van het levende verdoofde konijn om het stromingsgedrag van de bloedplaatjes te bestuderen. Het buikvlies is een dun vlies, dat via een kleine snede in de buikwand tezamen met een stukje darm naar buiten kan worden gebracht. In dit vlies kan dan met behulp van een speciale microscoop, het stromend bloed in de kleine vaten zichtbaar worden gemaakt. In deze vaten is het niet mogelijk om de bloedplaatjes waar te nemen, omdat ze worden overdekt met verschillende lagen van de grotere rode bloedcellen. Door de bloedplaatjes selectief te kleuren met de fluorescerende kleurstof acridine rood zijn ze, met behulp van een fluorescentie microscoop, eenvoudig te herkennen tussen de niet gekleurde rode cellen. Het kleuren van de bloedplaatjes gebeurde door een kleine hoeveelheid van de fluorescerende kleurstof direct in een bloedvat te spuiten.

$\mathrm{Na}$ het inspuiten werden er van geselecteerde venules via een videocamera, welke was geplaatst op de microscoop, opnames gemaakt. Deze opnames werden later uitgewerkt. Om onscherpte van het beeld door de snelstromende bloedplaatjes te voorkomen is gebruik gemaakt van belichting met telkens een korte flits. De bloedplaatjes worden als het ware "bevroren" in tijd en plaats. Wanneer we door de microscoop kijken is de plaats van het bloedplaatje in de lengte- en breedte richting van het vat goed te bepalen, terwijl het bepalen van de juiste diepte problemen geeft. De intensiteit van het fluorescerend plaatje neemt zeer snel af indien het zich niet meer bevindt in een gebied symmetrisch gelegen rondom het vlak van scherpstelling. Dit vlak, de zgn optische plak, maakt bepaling van de positie van het bloedplaatje in de diepte mogelijk. In alle experimenten wordt scherp gesteld in het midden van het vat.

Voor het uitwerken van de opnames werd van elk plaatje in de optische plak de afstand gemeten van bloedplaatje tot de linker vaatwand en de bijbehorende diameter van het vat. Hieruit werd de positie van het plaatje in het vat bepaald. Door nu de diameter in 20 segmenten te verdelen kan een concentratie verdeling worden verkregen. Deze concentratie verdeling geldt alleen voor de optische plak. Voor de concentratie verdeling over het gehele vat een is een correctie noodzakelijk.

\section{De resultaten}

Het eerste experimentele hoofdstuk (hoofdstuk 4) laat duidelijke verschillen zien tussen de concentratie verdeling van venulen en arteriolen. In arteriolen is in het centrale deel van het vat de concentratie lager dan in 
venulen, terwijl aan de wand het omgekeerde wordt gezien (figuur 4.3). Verder lijkt er dicht bij de wand in venulen een afstoting, een zgn. exclusie van bloedplaatjes te bestaan (figuur 4.4). Dit verschijnsel zou kunnen worden veroorzaakt door witte bloedcellen die langs de vaatwand rollen, een verschijnsel dat alleen in venulen wordt waargenomen. Echter, een vermindering van het aantal rollende witte bloedcellen m.b.v. een gesulfateerd dextraan deed niet de exclusie verdwijnen (figuur 4.5). Aangezien de exclusie eerder leek toe te nemen, werd ook de invloed van dit dextraan op de bloedplaatjes verdeling van arteriolen bestudeerd.

Dextranen zijn lange ketens van suiker-moleculen, die kunnen binden aan het oppervlak van o.a. bloedplaatjes, rode bloed cellen en vaatwandcellen. Verder kunnen ze rheologische parameters, bijv de stroperigheid van het bloed (viscositeit) en de vervormbaarheid van de rode bloed cellen (deformabiliteit) beinvloeden. Dextranen met een hoog molecuul gewicht zijn in staat om rode bloedcellen aan elkaar te laten hechten (aggregatie). In hoofdstuk 5 wordt de invloed van 3 verschillende dextranen, een neutraal geladen laag moleculair dextraan (Dx40), een neutraal hoog moleculair dextraan (Dx500) en een negatief geladen hoog moleculair dextraan (Dx500S) op de bloedplaatjes verdeling bestudeerd. De hoog moleculaire dextranen verhoogden in arteriolen de bloedplaatjes concentratie in het midden en verlaagden de concentratie aan de wand. In venulen werd hetzelfde effect gezien, doch veel minder uitgesproken. Laag moleculair dextraan had geen invloed op de verdeling van de bloedplaatjes in arteriolen, in venulen echter verlaagde dit dextraan de concentratie in het midden van het vat en verhoogde het aan de wand (figuur $5.1 \mathrm{t} / \mathrm{m} 5.3$ ). Daar de dextraan injecties geen invloed hadden op de stromingssnelheid van het bloed en op de viscositeit, is het waarschijnlijk de binding van dextranen aan de bloedplaatjes, rode bloed cellen en/of vaatwandcellen, die verantwoordelijk is voor de veranderde bloedplaatjes verdeling na dextraan injectie.

In hoofdstuk 6 wordt het snelheidsprofiel van venulen bestudeerd. Het snelheidsprofiel in water heeft de vorm van een parabool; lage snelheden in de buurt van de wand, oplopend tot de maximale snelheid in het centrum. Bloed is in vergelijking met water een substantie met andere kenmerken. De beschrijving van het snelheidprofiel in bloed is alleen goed mogelijk m.b.v. een twee vloeistoffen model; aan de wand bevindt zich een vloeistof met een andere samenstelling dan in de rest van het vat, waar de rode bloedcellen zich bevinden (RBC-kolom). Eerder onderzoek wees uit dat de vorm van het snelheidsprofiel in de RBC-kolom van arteriolen een afgeplatte parabool was. Opvallend was dat in venulen bleek dat de vorm 
van het snelheidsprofiel van de RBC-kolom niet verschillend was van een parabool.

In de kleine vaten bleek de verdeling van bloedcellen niet homogeen verdeeld over het vat (zie voor bloedplaatjes hoofdstuk 4). Rode bloedcellen stromen hoofdzakelijk in het midden van het vat. Aangezien daar de snellheid het hoogst is, betekent dit dat de gemiddelde rode bloed cel snelheid hoger is dan de gemiddelde snelheid van het bloed. Door dit verschil in gemiddelde snelheid is de gemiddelde verblijftijd van rode bloed cellen in een bepaald segment van het vat korter dan de gemiddelde verblijftijd van bloed. Om ophoping van rode bloed cellen te voorkomen moet de lokale rode bloed cel concentratie lager zijn dan voor homogeen verdeelde rode bloed cellen. In hoofdstuk 7 is dit zgn. Fåhraeus effect berekend voor bloedplaatjes. Hiervoor is gebruik gemaakt van de bloedplaatjes verdeling van arteriolen en venulen (hoofdstuk 4) en de snelheidsprofielen van arteriolen (eerder verricht onderzoek) en venulen (hoofdstuk 6). Berekeningen laten zien dat in arteriolen, waar de meeste plaatjes in de buurt van de wand stromen, er geen sprake is van een omgekeerd Fåhraeus effect, hetgeen men zou verwachten. In venulen echter, waar de meeste bloedplaatjes stromen op grotere afstand van de wand en met een hogere concentratie in het midden van het vat, is de gemiddelde bloedplaatjes snelheid hoger dan de gemiddelde snelheid van het bloed. Als gevolg hiervan zal de bloedplaatjes concentratie in de kleine vaten lager zijn dan verwacht op basis van een homogeen verdeelde concentratie van bloedplaatjes; er is een Făhraeus effect.

\section{Eindbeschouwing}

De bevindingen beschreven in dit proefschrift, n.l. de verschillen in bloedplaatjes concentratie verdeling en in snelheidsprofiel tussen venulen en arteriolen in vivo, kunnen niet eenvoudig worden verklaard door verschillen in parameters die het stromingsgedrag bepalen. Een effect van rollende witte bloedcellen in venulen op de bloedplaatjes verdeling is blijkbaar niet aanwezig. De invloed van rode bloedcellen op het stromingsgedrag van bloedplaatjes en de plaats van het vat in het vaatstelsel spelen mogelijk een veel belangrijkere rol. Het feit dat er sprake is van een bloedplaatjes exclusie aan de vaatwand in venulen en niet in arteriolen geeft een aanwijzing voor het bestaan van verschillen in vaatwand structuur en/of functionele verschillen tussen beide vaattypen. 


\section{PUBLICATIONS}

Papers

Woldhuis $\mathbb{B}$, GJ Tangelder, DW Slaaf and RS Reneman. Platelet distribution in venules of the rabbit mesentery. In: Manuskriptsammlung 13. Jahrestagung der Gesellschaft für Mikrozirkulation e.V., edited by M Wahl. München, 1989, pp $137-140$.

Woldhuis B, GJ Tangelder, DW Slaaf and RS Reneman. Concentration profile of blood platelets differs in arterioles and venules. Am J Physiol 262:H1217-1223, 1992.

Woldhuis B, GJ Tangelder, DW Slaaf and RS Reneman. Influence of dextrans on platelet distribution in arterioles and venules. Eur J Physiol, in press.

Reneman RS, B Woldhuis, MGA oude Egbrink, DW Slaaf and GJ Tangelder. Concentration and velocity profiles of blood cells in the microcirculation. In" Frontiers in cardivascular engineering. NHC Hwang, VT Turitto and MRT Yen (eds), Plemum, New York, 25-40, 1993.

Woldhuis B, GJ Tangelder, DW Slaaf and RS Reneman. Velocity profiles in rabbit mesenteric venules in vivo. Submitted Circ Res.

Slaaf DW, B Woldhuis, JW Corsel, RS Reneman and GJ Tangelder. Platelet Fahraeus. effect in arterioles and venules as calculated from measured velocity profiles and concentration distributions, Submitted of publication.

\section{Proceedings/Abstracts}

Woldhuis B, GJ Tangelder, DW Slaaf and RS Reneman. Platelet distribution in venules of the rabbit mesentery. Int J Microcirc Clin Exp 9:233, 1990.

Woldhuis B, GJ Tangelder, DW Slaaf and RS Reneman. Platelet distribution in venules and arterioles.Int J Microcirc Clin Exp 9:324, 1990

Woldhuis $B_{*}$ GJ Tangelder, DW Slaaf and RS Reneman. Platelet distribution differs in arterioles and in venules. Eur J Physiol (Pflügers Arch) 416:S6, 1990

Woldhuis B, GJ Tangelder, DW Slaaf and RS Reneman. Does leukocyte rolling explain the difference in platelet distribution between venules and arterioles. Int J Microcirc Clin Exp 9:suppl.1:167, 1990

Woldhuis B, GJ Tangelder, DW Slaaf and RS Reneman. Effect of high molecular weight dextran on platelet distribution in arterioles and venules. Int J Microcirc: Clin Exp $10: 375,1991$.

Woldhuis B, GJ Tangelder, DW Slaaf and RS Reneman. High molecular weight dextran influences platelet distribution in arterioles and venules. Thromb Haemostas $65: 983,1991$.

Tangelder GJ, B Woldhuis, DW SLaaf and RS Reneman. High molecular weight dextran increases platelet concentration in the center of arterioles and venules. 
Proceedings Fifth World Congress for Microcirculation, Louisville KY, USA 110, 1991.

Slaaf DW, B Woldhuis, GJ Tangelder and RS Reneman. Is an inverted Fahraeus effect present for blood platelets flowing in rabbit mesentery arterioles. Proceedings Fifth World Congress for Microcirculation, Louisville KY, USA 103, 1991.

Woldhuis B, GJ Tangelder, DW Slaaf and RS Reneman. Platelet distribution in arterioles is influenced by high molecular weight dextrans. Eur J Physiol (Pflügers Arch)421, R43, 1992.

Woldhuis B, GJ Tangelder, DW Slaaf and RS Reneman. Influence of neutral and negatively charged dextrans on platelet distribution in mesenteric arterioles of anesthetized rabbits. Int J Microcirc: Clin Exp 11 (suppl 1), 107, 1992.

Tangelder GJ, B Woldhuis, DW Slaaf and RS Reneman. Time dependent increase of red blood cell aggregation in anesthetized rabbits after intravenous injection of a 500 kD dextran. Int J Microcirc: Clin Exp 11 (suppl 1), 195, 1992.

Tangelder GJ, B Woldhuis, DW Slaaf and RS Reneman. Velocity profiles are less blunt in venules than in arterioles. Faseb J: 7(4), A904, 1993.

Slaaf DW, B Woldhuis, GJ Tangelder JW Corsel and RS Reneman. Fahraeus effect for blood platelets flowing in rabbit mesenteric arterioles and venules. Faseb J: 7(4), A903, 1993.

Woldhuis B, GJ Tangelder, DW Slaaf and RS Reneman. Velocity profiles and shear values in venules. Int J Microcirc: Clin $\operatorname{Exp} 13,52,1993$.

Reneman RS, B Woldhuis, DW Slaaf and GJ Tangelder. Platelet density and velocity profiles in arterioles and venules - estimation of wall shear rate (WSR) and wall shear stress (WSS). Berlin, 1993.

Tangelder GJ, B Woldhuis, DW Slaaf and RS Reneman. Flow behavior and hemodynamics in venules. Abstractbook of symposium on the venous microcirculation, New Orleans, 9, 1993. 


\section{DANKWOORD}

Na twee verhuizingen, twee bevallingen en een knie-letsel is het dan zover: het proefschrift is er!

De grootste bijdrage aan dit proefschrift is wellicht geleverd door mijn directe begeleider Prof.Dr. G.J.Tangelder. Geert-Jan, jouw enthousiasme voor het onderzoek en je prettige manier van omgaan zijn de drijfveren waardoor dit proefschrift tot stand is gekomen. Het vertrouwen dat je in mij had om, ondanks protesten, je fiat te geven het schrijven van dit proefschrift af te ronden in Krimpen a/d IJssel heb ik zeer gewaardeerd. Ik ben blij dat je nu, naast de in de winter zo bekende rode muts en dito das, sinds kort een toga mag dragen. Dick Slaaf is degene die het mathematische deel van dit proefschrift voor zijn rekening heeft genomen. Beste Dick, vol geduld trok je keer op keer weer tijd uit om mij, de dokter, de noodzakelijke wiskunde bij te brengen. Rob Reneman was altijd op de achtergrond aanwezig. Beste Rob, je verbaasde mij iedere keer weer door precies te weten welke problemen er gedurende een bepaald onderzoek een rol speelden.

Gedurende het onderzoek zijn het vooral de mensen op het Laboratorium voor Microcirculatie geweest die een grote bijdrage hebben geleverd: Sabrina Weymer met name tijdens de experimenten en later voor het maken van de figuren, Rinus Alewijnse voor het electro-technische gedeelte, Jan Corsel voor het schrijven van de benodigde soft-ware.

Zus Thomas wil ik bedanken voor haar gastvrijheid, ik kon er altijd logeren.

De leden van de beoordelingscommissie wil ik bedanken voor het kritisch doorlezen en beoordelen van het manuscript.

Zonder de hulp van diegenen die al die jaren de zorg voor mijn kinderen op zich hebben genomen had ik dit onderzoek niet kunnen verrichten. Gastmoeder Nicole Janssen nam de eerste jaren Anne liefdevol op in haar gezin. Na de geboorte van Erik namen Marije Bogmans en Wilma Dumond de zorg bij ons in huis over. Sonja Uktolzejo was aanwezig voor de kinderen als ik weer eens vanuit Krimpen a/d IJssel naar Maastricht was voor overleg. Voor mij stond er bij terugkomst altijd een heerlijke Indonesische maaltijd klaar. In geval van "acute oppasnood" deed ik nooit tevergeefs een beroep op mijn ouders en schoonouders.

Olaf, vele avonden hebben wij gepraat over een eerlijke verdeling van taken binnen ons gezin. De intentie was er wel, doch de daadwerkelijke 
uitvoering bleef achterwege. Desondanks was jij het toch die mij stimuleerde om vooral door te gaan. Anne en Erik, ik zal niet zeggen dat er nu meer tijd voor jullie is. Wel hoop ik dat jullie het later met mij eens zullen zijn dat het niet gaat om de kwantiteit, doch om de kwaliteit van het aanwezig zijn van de moeder.

Tenslotte wil ik iedereen bedanken die op de een of andere manier heeft meegewerkt aan het tot stand komen van dit proefschrift. 


\section{CURRICULUM VITAE}

De schrijfster van dit proefschrift werd geboren op 7 oktober 1957 te Veendam. Na het doorlopen van de middelbare school (atheneum B) werd in 1976 begonnen met de HBO opleiding Klinische Chemie aan de school voor laboratoriumpersoneel te Groningen. Na het behalen van het diploma in 1978 was zij als analiste werkzaam op de afdeling immunohematologie van het Centraal Laboratorium van de Bloedtransfusiedienst te Amsterdam. In 1979 ging zij geneeskunde studeren aan de Rijksuniversiteit Utrecht, welke in 1987 werd afgerond met het behalen van het arts examen. In hetzelfde jaar was zij enige maanden werkzaam als wetenschappelijk medewerkster bij de vakgroep hematologie van het Academisch Ziekenhuis Utrecht. Van 1988 tot 1992 was zij als assistent in opleiding verbonden aan de vakgroep Fysiologie van de Rijksuniversiteit Limburg, alwaar het in dit proefschrift beschreven onderzoek werd verricht. Sinds 1993 is zij werkzaam als verzekeringsgeneeskundige bij een bedrijfsvereniging. 